\title{
On the crystalline period map
}

\author{
A. BeiLINSON*
}

To Irene

\begin{abstract}
1991 Mathematics Subject Classification: Primary 14F30, 14F40; secondary 14F20.

KEYWORDS AND PHRASES: p-adic periods, log crystalline cohomology, h-topology, alterations.
\end{abstract}

This article is a direct continuation of [B]. It contains a simple proof of comparison theorems in $p$-adic Hodge theory (the Fontaine-Jannsen conjecture). Different proofs were found earlier by Faltings, Niziol, and Tsuji, the case of open varieties treated by Yamashita. An alternative approach, based on an identification of the log crystalline cohomology for lci maps with the noncompleted (for the Hodge filtration) derived de Rham complex, was developed by Bhatt [Bh2].

Let $\mathcal{V}_{\bar{K}}$ be the category of algebraic varieties over an algebraic closure $\bar{K}$ of a $p$-adic field $K$. Our principal character is a natural h-sheaf of dg algebras $\mathcal{A}_{\text {crys }}$ on $\mathcal{V} r_{\bar{K}}$ equipped with a Frobenius action; let $X \mapsto$ $R \Gamma_{\text {crys }}(X):=R \Gamma\left(X_{\mathrm{h}}, \mathcal{A}_{\text {crys }}\right), X \in \mathcal{V} a r_{\bar{K}}$, be the corresponding cohomology theory. We construct $\mathcal{A}_{\text {crys }}$ applying h-localization procedure, as in [B] 2.6, to the absolute (i.e., relative to $\mathbb{Z}_{p}$ ) $\log$ crystalline cohomology of $\log O_{\bar{K}^{-}}$ schemes coming from semi-stable pairs. The algebra $\mathcal{A}_{\text {crys }}(\operatorname{Spec} \bar{K})$ equals the Fontaine ring $A_{\text {crys }}$. The crystalline $p$-adic Poincaré lemma asserts that $\mathcal{A}_{\text {crys }} \otimes{ }^{L} \mathbb{Z} / p^{n}$ is a constant h-sheaf with fiber $A_{\text {crys }} / p^{n}$. It yields a morphism of dg algebras $\rho_{\text {crys }}: R \Gamma_{\text {crys }}(X) \rightarrow R \Gamma_{\text {ét }}\left(X, \mathbb{Z}_{p}\right) \otimes_{\mathbb{Z}_{p}} \mathrm{~A}_{\text {crys }}$. This crystalline period map becomes an isomorphism after $\mathrm{B}_{\text {crys }}$-localization, which implies the Fontaine-Jannsen conjecture.

The article runs as follows: $\S 1$, that takes most of the pages, is an exposition of log crystalline cohomology and Hyodo-Kato theory. In $\S 2$ we define $\mathcal{A}_{\text {crys }}$ and prove the crystalline $p$-adic Poincaré lemma. Like its Hodgecompleted counterpart of [B] 3.3, the Poincaré lemma comes from Bhatt's

\footnotetext{
${ }^{*}$ The author was supported in part by NSF grant DMS-1001660.
} 
theorem [B] 4.3, [Bh1] 1.1, but to deduce it we use the conjugate spectral sequence instead of the Hodge-de Rham one. We also define the Hyodo-Kato cohomology $^{1} R \Gamma_{\mathrm{HK}}(X)$ and identify $R \Gamma_{\text {crys }}(X) \otimes \mathbb{Q}$ with its twisted form; since, by Hyodo-Kato, another twist of $R \Gamma_{\mathrm{HK}}(X)$ equals $R \Gamma_{\mathrm{dR}}(X)$, we get control on $R \Gamma_{\text {crys }}(X) \otimes \mathbb{Q}$. In $\S 3$ we prove the above isomorphism assertion for $\rho_{\text {crys }}$, deduce from it the Fontaine and Fontaine-Jannsen conjectures $\mathrm{C}_{\text {crys }}$, $\mathrm{C}_{\mathrm{st}}, \mathrm{C}_{\mathrm{pst}}$, and show that the Hodge filtration completion of $\rho_{\text {crys }}$ equals the period map $\rho_{\mathrm{dR}}$ from $[\mathrm{B}] 3.5$.

I am very grateful to Bhargav Bhatt, Volodya Drinfeld, Luc Illusie, Kazuya Kato, and the referee for valuable comments, suggestions, and corrections.

\section{Log crystalline cohomology: a review}

The section can be divided into five parts:

(i) Log preliminaries (1.1-1.4). The main reference for log schemes is [K1]; for an expanded exposition, see [GR] Ch. 3, 7 and [Og1]. We show that the key existence result for pd-envelopes, proved in [K1] 5.4 for fine log schemes, remains true for integral quasi-coherent log schemes, which is our preferred log crystalline setting.

(ii) Log crystalline basics (1.5-1.12). One finds a concise exposition of the basics of Berthelot's theory [Ber] in [BhdJ]; we discuss in similar vein Kato's log crystalline theory $[\mathrm{K} 1] \S \S 5-6,[\mathrm{HK}] \S 2$, including the comparison theorem of Illusie and Olsson.

(iii) Frobenius log crystals and the Hyodo-Kato theory (1.13-1.16). We deduce the key global results of Hyodo-Kato directly from the identification of nondegenerate Frobenius crystals up to isogeny on $\operatorname{Spec}\left(O_{K} / p\right)$ with $(\varphi, N)$ modules (cf. [Fa] $\S 2,[\mathrm{Ol} 2] 5.3$ ). The original approach of [HK] $\S 5$ (see also [Ill3] §7) is local and uses de Rham-Witt complexes. The core of both arguments goes back to Dwork ([Ka] 3.1).

(iv) The Fontaine rings and absolute crystalline cohomology of log schemes over $O_{\bar{K}}(1.17-1.18)$. This is an exposition of [K2] $\S \S 3-4$.

(v) Log de Rham complex in characteristic 0 (1.19). This is an exposition of a theorem of Ogus [Og2] Th. 1.3.

1.1. Log schemes. A log scheme is denoted as $(Z, \mathcal{M})=(Z, \mathcal{M}, \alpha), Z$ is the underlying scheme, $\mathcal{M}$ is the (étale) monoid sheaf, $\alpha: \mathcal{M} \rightarrow \mathcal{O}_{Z}$ is

\footnotetext{
${ }^{1}$ It is constructed by the same procedure as $R \Gamma_{\text {crys }}(X)$ using the Hyodo-Kato cohomology of log schemes coming from semi-stable pairs as the input.
} 
the log structure map. A chart, or $M$-chart, for it is a homomorphism of monoids $c_{M}: M \rightarrow \Gamma(Z, \mathcal{M})$ (the chart map) which yields an isomorphism $M_{Z}^{a} \stackrel{\sim}{\rightarrow} \mathcal{M}$; here $M_{Z}$ is the constant sheaf of monoids on $X_{\text {ét }}$ with fibers $M$ and $M_{Z}^{a}=\left(M_{Z}^{a}, \alpha\right)$ is the $\log$ structure generated by the prelog one $\alpha c_{M}: M_{Z} \rightarrow \mathcal{O}_{Z}$. A chart is integral if $M$ is integral (i.e., the canonical map $M \rightarrow M^{\mathrm{gr}}$ - the group completion of $M$ - is injective) and fine if $M$ is fine, i.e., integral and finitely generated.

We say that $(Z, \mathcal{M})$ is $\log$ affine if $Z$ is affine and $\Gamma(Z, \mathcal{M})$ generates $\mathcal{M}{ }^{2}$ quasi-coherent ([K1] 2.1) if it admits a chart étale locally, integral ${ }^{3}$ ([K1] 2.2) if $\mathcal{M}$ is integral, and fine ([K1] 2.3) if it admits a fine chart étale locally. We identify schemes with log schemes with trivial log structure, and write $X$ for $\left(X, \mathcal{O}_{X}^{\times}\right)$.

The category of log schemes has finite inverse limits; the underlying scheme functor $(Z, \mathcal{M}) \mapsto Z$ commutes with inverse limits ([K1] 1.6).

Let $f:(Z, \mathcal{M}) \rightarrow(S, \mathcal{L})$ be a map of log schemes. A chart for it consists of charts $M \rightarrow \Gamma(Z, \mathcal{M}), L \rightarrow \Gamma(S, \mathcal{L})$ and a map of monoids $L \rightarrow M$ such that the evident diagram commutes. The chart is fine if both $M, L$ are fine.

Proposition. If $(S, \mathcal{L})$ is fine, $(Z, \mathcal{M})$ is integral quasi-coherent, then $f$ admits a chart étale locally. If $(Z, \mathcal{M})$ is also fine, then $f$ admits a fine chart étale locally.

Proof. The second claim is [K1] 2.10. We modify Kato's proof to cover the first claim. Let us construct an étale neighborhood with a chart. Let $\left\{\ell_{i}\right\}_{i \in I}$ be a finite subset of $\mathcal{L}_{s}$ that generates $\mathcal{L}_{s} / \mathcal{O}_{S, s}^{\times}$. Let $K \subset L \subset \mathbb{Z}^{I}$ be the preimages of $\mathcal{O}_{S, s}^{\times}$and $\mathcal{L}_{s}$ by the map $\mathbb{Z}^{I} \rightarrow \mathcal{L}_{s}^{\text {gr }}, e_{i} \mapsto \ell_{i}$. Let $\left\{k_{j}\right\}_{j \in J}$ be a base of $K$, so $k_{j}=\Sigma n_{j i} e_{i}-\Sigma n_{j i}^{\prime} e_{i}$, where $n_{j i}, n_{j i}^{\prime} \in \mathbb{N}$. Then $e_{i}, k_{j}$, and $-k_{j}$ generate $L$ as a monoid, with $k_{j}+\Sigma n_{j i}^{\prime} e_{i}=\Sigma n_{j i} e_{i}, k_{j}+\left(-k_{j}\right)=0$ being the full set of relations as of integral monoid. As in [K1] 2.10, after localizing $S$, the map $L \rightarrow \mathcal{L}_{s}$ lifts to a chart $c_{L}: L \rightarrow \Gamma(S, \mathcal{L})$.

Pick any $z \in Z$. Let us construct its étale neighborhood with a chart for $f$. Localizing $Z$, we can assume that $(Z, \mathcal{M})$ has chart $M^{\prime \prime}$ and $f^{*}\left(\ell_{i}\right)=m_{i} g_{i}$, where $m_{i} \in M^{\prime \prime}, g_{i} \in \Gamma\left(Z, \mathcal{O}_{Z}^{\times}\right)$. Set $h_{j}:=f^{*}\left(c_{L}\left(k_{j}\right)\right) \in \Gamma\left(Z, \mathcal{O}_{Z}^{\times}\right)$. Notice that $M^{\prime}:=M^{\prime \prime} \oplus \Gamma\left(Z, \mathcal{O}_{Z}^{\times}\right)$, equipped with an evident chart map $c_{M^{\prime}}$, is another chart for $\mathcal{M}$. The elements $\left(0, h_{j}\right)+\Sigma n_{j i}^{\prime}\left(m_{i}, g_{i}\right)$ and $\Sigma n_{j i}\left(m_{i}, g_{i}\right)$ of $M^{\prime}$ are identified by $c_{M^{\prime}}$. Let $M$ be the integral monoid quotient of $M^{\prime}$

\footnotetext{
${ }^{2}$ I.e., every local section of $\mathcal{M}$ is the product of a global section and an invertible function.

${ }^{3}$ In present article "integral" in the sense of "reduced irreducible" (scheme) is never used.
} 
modulo the relations $\left(0, h_{j}\right)+\Sigma n_{j i}^{\prime}\left(m_{i}, g_{i}\right)=\Sigma n_{j i}\left(m_{i}, g_{i}\right)$. Then $c_{M^{\prime}}$ factors through $c_{M}: M \rightarrow \Gamma(Z, \mathcal{M})$, which is again a chart, and we have a map of monoids $L \rightarrow M$ which assigns to $e_{i}, k_{j}$ the images of $\left(m_{i}, g_{i}\right),\left(0, h_{j}\right)$ in $M$. This is the promised chart for $f$.

Exercises. (i) Every quasi-coherent $(Z, \mathcal{M})$ admits the maximal closed integral log subscheme $(Z, \mathcal{M})^{\text {int }}$. The $\log$ scheme $(Z, \mathcal{M})^{\text {int }}$ is quasi-coherent, and the functor $(Z, \mathcal{M}) \mapsto(Z, \mathcal{M})^{\text {int }}$ is right adjoint to the embedding of the category of quasi-coherent integral log schemes into that of quasi-coherent $\log$ schemes.

(ii) If $\left(Z_{i}, \mathcal{M}_{i}\right), i=1,2$, are integral quasi-coherent log schemes over a fine $\log$ scheme $(S, \mathcal{L})$, then $\left(Z_{1}, \mathcal{M}_{1}\right) \times_{(S, \mathcal{L})}\left(Z_{2}, \mathcal{M}_{2}\right)$ is quasi-coherent. ${ }^{4}$

(iii) Suppose $(T, \mathcal{N})$ is integral and $(Z, \mathcal{M}) \hookrightarrow(T, \mathcal{N})$ is an exact closed embedding defined by a nil ideal $\mathcal{J} \subset \mathcal{O}_{T}$. If $Z$ is affine, then for any $m \in \Gamma(Z, \mathcal{M})$ the set of its liftings to $\Gamma(T, \mathcal{N})$ is a $\Gamma\left(T,(1+\mathcal{J})^{\times}\right)$-torsor. ${ }^{5}$ $(T, \mathcal{N})$ is quasi-coherent if (and only if) such is $(Z, \mathcal{M}) .{ }^{6}$

Remarks. (i) Let $f:(Z, \mathcal{M}) \rightarrow(S, \mathcal{L})$ be a map of fine log schemes; suppose $S$ is affine, $Z / S$ is finitely presented. Then there is $f^{\prime}:\left(Z^{\prime}, \mathcal{M}^{\prime}\right) \rightarrow\left(S^{\prime}, \mathcal{L}^{\prime}\right)$ having the same properties with $S^{\prime}$ affine of finite type over $\mathbb{Z}$, and a map $(S, \mathcal{L}) \rightarrow\left(S^{\prime}, \mathcal{L}^{\prime}\right)$ such that $\mathcal{L}$ is the pullback of $\mathcal{L}^{\prime}$ and $f$ isomorphic to the pullback of $f^{\prime}$; for $f \log$ smooth, one can find $\log$ smooth $f^{\prime}$.

(ii) Suppose $f$ as in (i) is $\log$ smooth, $Z$ is affine, and we have a closed exact embedding $(S, \mathcal{L}) \hookrightarrow(T, \mathcal{N})$ defined by a nil ideal $\mathcal{J} \subset \mathcal{O}_{T}$; then $f$ is the pullback of some $\log$ smooth $f_{T}:\left(Z_{T}, \mathcal{M}_{T}\right) \rightarrow(T, \mathcal{N})$ defined uniquely up to an isomorphism. ${ }^{7}$

We denote by $\mathbb{A}_{(S, \mathcal{L})}^{1}$ and $\mathbb{A}_{(S, \mathcal{L})}^{(1)}$ the affine line and the logarithmic affine line over a $\log$ scheme $(S, \mathcal{L})$. Both equal $\operatorname{Spec} \mathcal{O}_{S}[t]$ as schemes, the log structures are generated by, respectively, $\mathcal{L}$ and $\mathcal{L} \oplus \mathbb{N}$, the latter log structure map sends $n \in \mathbb{N}$ to $t^{n}$. For a $\log (S, \mathcal{L})$-scheme $(Z, \mathcal{M})$, a map from it to $\mathbb{A}_{(S, \mathcal{L})}^{1}$ is (the same as) a section of $\mathcal{O}_{Z}$, a map to $\mathbb{A}_{(S, \mathcal{L})}^{(1)}$ is a section of $\mathcal{M}$. The group scheme $\mathbb{G}_{a}$ acts on $\mathbb{A}_{(S, \mathcal{L})}^{1}$ by translations, $\mathbb{G}_{m}$ acts on $\mathbb{A}_{(S, \mathcal{L})}^{(1)}$ by

\footnotetext{
${ }^{4}$ Hint: Use the fact that étale locally both projections $\left(Z_{i}, \mathcal{M}_{i}\right) \rightarrow(S, \mathcal{L})$ admit charts with the same $L$ (follows from the proof of the proposition).

${ }^{5}$ Hint: $T$ is affine and $H^{>0}\left(T_{\text {ét }},(1+\mathcal{J})^{\times}\right)=0$.

${ }^{6}$ Hint: If $Z$ is affine and $M \rightarrow \Gamma(Z, \mathcal{M})$ is a chart for $\mathcal{M}$, then $M \times_{\Gamma(Z, \mathcal{M})}$ $\Gamma(T, \mathcal{N}) \rightarrow \Gamma(T, \mathcal{N})$ is a chart for $\mathcal{N}$.

${ }^{7} \mathrm{By}(\mathrm{i})$, we are reduced to the situation when $\mathcal{J}$ is nilpotent; now the assertion is [K1] 3.14 .
} 
homotheties. For a (possibly infinite) set $I$ we denote by $\mathbb{A}_{(S, \mathcal{L})}^{I}, \mathbb{A}_{(S, \mathcal{L})}^{(I)}$ the fiber products of $I$ copies of $\mathbb{A}_{(S, \mathcal{L})}^{1}, \mathbb{A}_{(S, \mathcal{L})}^{(1)}$.

1.2. Log pd-schemes. For us, a log $p d$-scheme is a log scheme $\left(T, \mathcal{M}_{T}\right)$ equipped with a pd-ideal $\left(\mathcal{J}_{T}, \delta\right)$ (so $\mathcal{J}_{T}$ is a quasi-coherent ideal in $\mathcal{O}_{T}$, $\delta$ a pd structure on it). A pd-thickening of a $\log$ scheme $(Z, \mathcal{M})$ is an exact closed embedding ([K1] 3.1) of $(Z, \mathcal{M})$ into a log pd-scheme as above such that $\mathcal{O}_{Z} \stackrel{\sim}{\rightarrow} \mathcal{O}_{T} / \mathcal{J}_{T}$. Log pd-schemes, i.e., pd-thickenings, form naturally a category. We often abbreviate $\left(T, \mathcal{M}_{T}, \mathcal{J}_{T}, \delta\right)$ to $\left(T, \mathcal{M}_{T}, \mathcal{J}_{T}\right)$; a pd-thickening as above is denoted by $\left(Z, T, \mathcal{M}_{T}\right)$ or simply $(Z, T)$.

Let $S^{\sharp}=(S, \mathcal{L}, \mathcal{I}, \gamma)$ be a log pd-scheme with $p \in \mathcal{O}_{S}$ nilpotent, $p^{n}=0$. Below a $\log$ scheme over $S^{\sharp}$, or a $\log S^{\sharp}$-scheme, means a log scheme $(Z, \mathcal{M})$ over $(S, \mathcal{L})$ such that $\gamma$ extends to $Z$ (i.e., to $\left.\mathcal{I} \mathcal{O}_{Z}\right)$. A $\log p d-S^{\sharp}$-scheme is a $\log S^{\sharp}$-scheme $\left(T, \mathcal{M}_{T}\right)$ equipped with a pd-ideal $\left(\mathcal{J}_{T}, \delta\right)$ such that $\gamma$ and $\delta$ extend to a pd structure on $\mathcal{J}_{T}+\mathcal{I} \mathcal{O}_{T}$. We can consider $p d$-S $S^{\sharp}$-thickenings of $(Z, \mathcal{M})$, etc. Notice that $\mathcal{J}_{T}$ is a nil ideal (since for $a \in \mathcal{J}_{T}$ one has $a^{p} \in p \mathcal{J}_{T}$ and $\left.p^{n}=0\right)$. Thus pd- $S^{\sharp}$-thickenings of $\left(Z, \mathcal{M}_{Z}\right)$ have $Z_{\text {ét-local nature. }}$

The categories of log pd-schemes, $\log S^{\sharp}$-schemes, and $\log$ pd- $S^{\sharp}$-schemes have finite inverse limits. For a group scheme $G$ we denote by $G^{\sharp}$ its pdenvelope at $1 \in G$; this is a group pd-scheme. One has $\mathbb{G}_{m}^{\sharp}((Z, T))=$ $\Gamma\left(T,\left(1+\mathcal{J}_{T}\right)^{\times}\right)$.

1.3. Log pd-envelopes. Suppose $S^{\sharp}$ from 1.2 is such that $(S, \mathcal{L})$ is quasicoherent. Let $\mathcal{C}_{S^{\sharp}}$ be the category whose objects are locally closed embeddings ${ }^{8} i_{Y}:(Z, \mathcal{M}) \hookrightarrow(Y, \mathcal{N})$ of $\log S^{\sharp}$-schemes such that $(Z, \mathcal{M})$ is integral quasi-coherent, $(Y, \mathcal{N})$ is quasi-coherent (the morphisms are maps of $(Y, \mathcal{N})$ 's that preserve $(Z, \mathcal{M})$ 's). Let $\mathcal{T}_{S^{\sharp}}$ be the category of $\log \mathrm{pd}-S^{\sharp}$-thickenings $(Z, T)$ as in 1.2 such that $\left(T, \mathcal{M}_{T}\right)$, hence $(Z, \mathcal{M})$, is integral quasi-coherent.

Theorem. The evident functor $\mathcal{T}_{S^{\sharp}} \rightarrow \mathcal{C}_{S^{\sharp}}$ (forgetting of the pd structure on $\mathcal{J}_{T}$ ) admits a right adjoint.

The theorem says that every $i_{Y}$ in $\mathcal{C}_{S^{\sharp}}$ admits a factorization

$$
(Z, \mathcal{M}) \stackrel{i_{T}}{\hookrightarrow}\left(T, \mathcal{M}_{T}\right) \stackrel{r}{\rightarrow}(Y, \mathcal{N})
$$

with $(Z, T) \in \mathcal{T}_{S^{\sharp}}$ which is universal. Such $(Z, T)$ is called the $p d$ - $S^{\sharp}$-envelope of $i_{Y}$.

\footnotetext{
${ }^{8}$ I.e., $(Z, \mathcal{M})$ is a closed $\log$ subscheme of an open part of $(Y, \mathcal{N})$.
} 
Proof. Let us construct $i_{T}$. When $(Z, \mathcal{M})$ is fine, $(Y, \mathcal{N})$ is coherent, this was done by Kato [K1] 5.4. The idea of the general argument: after étale localization and adding extra polynomial variables to $Y$, one can realize $i_{Y}$ as a filtered inverse limit of embeddings of fine log schemes, which brings $i_{T}$ by loc. cit.; the extra polynomial variables are then factored off. Here are the details:

(a) Suppose we have some factorization $i_{T}$ as in (1.3.1) and $U \in Y_{\text {ét. }}$. Consider the $U$-localizations $i_{U}:\left(Z_{U}, \mathcal{M}_{Z_{U}}\right):=(Z, \mathcal{M}) \times_{Y} U \hookrightarrow\left(U, \mathcal{N}_{U}\right)$, $\left(T_{U}, \mathcal{M}_{T_{U}}\right):=\left(T, \mathcal{M}_{T}\right) \times_{Y} U$. If $(Z, T)$ is the pd- $S^{\sharp}$-envelope of $i_{Y}$, then $\left(T_{U}, \mathcal{M}_{T_{U}}\right)$ is the pd-S $S^{\sharp}$-envelope of $i_{U}$. The converse is true if $U$ is a covering of $Y$.

(b) The assertion of the theorem has étale local nature: If $U$ is an étale covering of $Y$ and $i_{U}:\left(Z_{U}, \mathcal{M}_{Z_{U}}\right) \hookrightarrow\left(U, \mathcal{N}_{U}\right)$ admits the pd-S $S^{\sharp}$-envelope, then $i$ admits the pd- $S^{\sharp}$-envelope. Proof: Let us construct the pd- $S^{\sharp}$-envelope $(Z, T)$. We can assume that $Z, Y, U$ are affine schemes, $Z$ is closed in $Y$. By (a), if $U^{\prime} \in Y_{\text {ét }}$ admits a morphism to $U$, then $i_{U^{\prime}}$ admits the pd- $S^{\sharp}$-envelope. Take $U^{\prime}:=U \times_{Y} U$; let $\left(T_{U}, \mathcal{M}_{T_{U}}\right),\left(T_{U^{\prime}}, \mathcal{M}_{T_{U^{\prime}}}\right)$ be the pd-envelopes. Then $T_{U^{\prime}}$ is an equivalence relation on $T_{U}$ by (a), and the schemes $T_{U}, T_{U^{\prime}}$ are affine (since such are $Z_{U}, Z_{U^{\prime}}$ ). Define $T$ as the affine scheme quotient of $T_{U}$ modulo the equivalence relation $T_{U^{\prime}}$, i.e., $T$ is an affine scheme with $\Gamma\left(T, \mathcal{O}_{T}\right)$ equal to the equalizer of $\Gamma\left(T_{U}, \mathcal{O}_{T_{U}}\right) \rightrightarrows \Gamma\left(T_{U^{\prime}}, \mathcal{O}_{T_{U^{\prime}}}\right)$. It is a pd-thickening of $Z$ over $Y$ relative to $(S, \mathcal{I}, \gamma)$. The map $T_{U} \rightarrow T$ is an étale covering and $T_{U^{\prime}}=T_{U} \times_{T} T_{U}$ (indeed, since our claim has étale local nature with respect to $Y$, to check it we can assume that $i$ admits pd- $S^{\sharp}$-envelope, and then it follows by (a)). We define the log structure $\mathcal{M}_{T}$ by the étale descent from $\mathcal{M}_{T_{U}}$. Then $(Z, T)$ is the pd- $S^{\sharp}$-envelope of $i_{Y}$ by (a).

(c) The pd- $S^{\sharp}$-envelope of $i_{Y}$ equals that of $(Z, \mathcal{M}) \hookrightarrow(Y, \mathcal{N})^{\text {int }}$, so we can assume that $(Y, \mathcal{N})$ is integral. The pd- $S^{\sharp}$-envelope of $i_{Y}$ equals its pd$Y^{\sharp}$-envelope, where $Y^{\sharp}=\left(Y, \mathcal{N}, \mathcal{I} \mathcal{O}_{Y}\right)$, so we can assume that $(Y, \mathcal{N})=$ $(S, \mathcal{L})$. By (b), we can assume that $Z, Y$ are affine, $Z$ closed in $Y$, and one has integral monoids $M, N$ and charts $M \rightarrow \Gamma(Z, \mathcal{M}), N \rightarrow \Gamma(Y, \mathcal{N})$.

Pick a set of generators $\left\{m_{i}\right\}_{i \in I}$ of $M$. Let $i_{V}:(Z, \mathcal{M}) \hookrightarrow(V, \mathcal{K}):=$ $\mathbb{A}_{(Y, \mathcal{N})}^{(I)}$ be the lifting of $i_{Y}$ that corresponds to $\left\{m_{i}\right\}$ (see 1.1). Let us show that $i_{V}$ admits a $p d-Y^{\sharp}$-envelope $\left(\tilde{T}, \mathcal{M}_{\tilde{T}}\right)$.

For a subset $J$ of $I$ let $M(J)$ be the submonoid of $M$ generated by $m_{j}, j \in J$, and $\mathcal{M}(J) \rightarrow \mathcal{O}_{Z}$ be the log structure generated by prelog one $M(J)_{Z} \rightarrow \mathcal{O}_{Z}$; there is an evident morphism $\mathcal{M}(J) \rightarrow \mathcal{M}$. Let $\mathcal{S}$ be the set of triples $s=\left(N^{s}, I^{s}, \gamma^{s}\right), N^{s}$ is a finitely generated submonoid of $N, I^{s}$ is a finite subset of $I, \gamma^{s}: N^{s} \rightarrow \Gamma\left(Z, \mathcal{M}\left(I^{s}\right)\right)$ is a map of monoids which lifts the composition $N^{s} \subset N \rightarrow \Gamma(Y, \mathcal{N}) \rightarrow \Gamma(Z, \mathcal{M})$. The natural order on $\mathcal{S}$ 
makes it a directed set, and for every finitely generated $N^{\prime} \subset N$ and finite $I^{\prime} \subset I$ one can find $s \in \mathcal{S}$ with $N^{\prime} \subset N^{s}, I^{\prime} \subset I^{s}$.

For $s \in \mathcal{S}$ let $\mathcal{N}^{s} \rightarrow \mathcal{O}_{Y}, \mathcal{K}^{s} \rightarrow \mathcal{O}_{V}$ be the log structures generated by the prelog ones $N_{Y}^{s} \rightarrow \mathcal{O}_{Y}, N_{V}^{s} \oplus \mathbb{N}\left[I^{s}\right]_{V} \rightarrow \mathcal{O}_{V}$; set $\mathcal{M}^{s}:=\mathcal{M}\left(I^{s}\right)$. The map of monoids $N^{s} \oplus \mathbb{N}\left[I^{s}\right] \rightarrow \Gamma\left(Z, \mathcal{M}^{s}\right)$, which is $\gamma^{s}$ on the first component and the evident map on the second one, extends $Z \hookrightarrow V$ to a closed embedding of fine $\log$ schemes $i_{V}^{s}:\left(Z, \mathcal{M}^{s}\right) \hookrightarrow\left(V, \mathcal{K}^{s}\right)$. Let $\left(Z, \mathcal{M}^{s}\right) \hookrightarrow\left(\tilde{T}^{s}, \mathcal{M}_{\tilde{T}^{s}}\right) \rightarrow\left(V, \mathcal{K}^{s}\right)$ be its pd- $Y^{s \sharp}$-envelope, where $Y^{s \sharp}=\left(Y, \mathcal{N}^{s}, \mathcal{I} \mathcal{O}_{Y}\right)$. By [K1] 5.4, it exists and $\tilde{T}^{s}$ is affine. When $s \in \mathcal{S}$ varies, the pd-envelopes form an $\mathcal{S}$-projective system. Its projective limit is the promised pd- $Y^{\sharp}$-envelope $\left(\tilde{T}, \mathcal{M}_{\tilde{T}}\right)$ of $i_{V}$. Indeed, $\left(\tilde{T}, \mathcal{M}_{\tilde{T}}\right)$ is evidently integral, hence it is quasi-coherent by Exercise (iii) in 1.1 , so $(Z, \tilde{T}) \in \mathcal{T}_{Y^{\sharp}}$, and the universality property is evident.

(d) As in $1.1,(V, \mathcal{K})$ carries the $\mathbb{G}_{m}^{I}$-action. ${ }^{9}$ By the universality property, the group pd-scheme $\mathbb{G}_{m}^{\sharp I}$ (see 1.2$)$ acts on $\left(\tilde{T}, \mathcal{M}_{\tilde{T}}\right)$. Let $\left(T, \mathcal{M}_{T}\right)$ be the quotient $\log \operatorname{pd}-Y^{\sharp}$-scheme, so $T=\operatorname{Spec}\left(\mathcal{O}(\tilde{T})^{\mathbb{G}_{m}^{\sharp I}}\right)$ and $\mathcal{M}_{T}$ is the sheaf of $\mathbb{G}_{m}^{\sharp I}$-invariant sections of $\mathcal{M}_{\tilde{T}}$. Let us show that the evident map $i_{T}$ : $(S, \mathcal{M}) \rightarrow\left(T, \mathcal{M}_{T}\right)$ is the pd- $Y^{\sharp}$-envelope of $i_{Y}$.

By construction, $\left(T, \mathcal{M}_{T}\right)$ is a $\log \operatorname{pd}-Y^{\sharp}$-scheme with $T$ affine and $\mathcal{M}_{T}$ integral. $i_{T}$ is a closed embedding of $\log$ schemes since $(S, \mathcal{M})$ is a closed $\log$ subscheme of $(Y, \mathcal{N})$. It is exact since $i_{V}$ is exact. By Exercise (iii) in 1.1, $\left(T, \mathcal{M}_{T}\right)$ is quasi-coherent. Thus $(Z, T) \in \mathcal{T}_{Y^{\sharp}}$. It remains to prove the universality property.

Suppose $\left(Z^{\prime}, T^{\prime}\right) \in \mathcal{T}_{Y^{\sharp}}$ is such that the structure map $\left(T^{\prime}, \mathcal{M}_{T^{\prime}}^{\prime}\right) \rightarrow$ $(Y, \mathcal{N})$ sends $\left(Z^{\prime}, \mathcal{M}^{\prime}\right)$ to $(Z, \mathcal{M})$; we want to show that there is a unique $\mathcal{T}_{Y^{\sharp-}}$ morphism $\left(Z^{\prime}, T^{\prime}\right) \rightarrow(Z, T)$. We can assume that $Z^{\prime}$ is affine, so the set of $\mathcal{T}_{Y^{\sharp} \text {-morphisms }}\left(Z^{\prime}, T^{\prime}\right) \rightarrow(Z, \tilde{T})$ is a $\mathbb{G}_{m}^{\sharp I}\left(\left(Z^{\prime}, T^{\prime}\right)\right)$-torsor (see Exercise (iii) in 1.1). So there is a unique $\left(Z^{\prime}, T^{\prime}\right) \rightarrow(Z, T)$ that can be lifted to $(Z, \tilde{T})$. To finish the proof, it suffices to check that every $s:(Z, T) \rightarrow(Z, \tilde{T})$ is a section of the projection $p:(Z, \tilde{T}) \rightarrow(Z, T)$. Now $p s=\operatorname{id}_{(Z, T)}$ amounts to $p s p=p$, which is clear since $s p=g \cdot \operatorname{id}_{(Z, \tilde{T})}$ for some $g \in \mathbb{G}_{m}^{\sharp I}((Z, \tilde{T}))$.

1.4. Log pd-smooth thickenings. An object $(Z, T)$ of $\mathcal{T}_{S^{\sharp}}$ (see 1.3) is said to be $p d-S^{\sharp}$-smooth if the next condition is satisfied: Suppose we have $\left(Z^{\prime}, T^{\prime}\right) \in$ $\mathcal{T}_{S^{\sharp}}$ such that $Z^{\prime}$ is affine (as a plain scheme); then any map of $\log (S, \mathcal{L})$ schemes $\left(Z^{\prime}, \mathcal{M}^{\prime}\right) \rightarrow(Z, \mathcal{M})$ can be extended to a morphism $\left(Z^{\prime}, T^{\prime}\right) \rightarrow$ $(Z, T)$ in $\mathcal{T}_{S^{\sharp}}$.

\footnotetext{
${ }^{9}$ For $g=\left(g_{i}\right) \in \mathbb{G}_{m}^{I}$ one has $g^{*}\left(t_{i}\right)=g_{i}^{-1} t_{i}$.
} 
Remarks. (i) Suppose $(Z, \mathcal{M})$ is an integral quasi-coherent log $S^{\sharp}$-scheme, and we have its closed embedding $i$ into $\mathbb{A}_{(S, \mathcal{L})}^{I} \times{ }_{(S, \mathcal{L})} \mathbb{A}_{(S, \mathcal{L})}^{(J)}$, where $I, J$ are two sets (see 1.1). Then the pd- $S^{\sharp}$-envelope $\left(P, \mathcal{M}_{P}\right)$ of $i$ is $\mathrm{pd}-S^{\sharp}$-smooth. We call such pd- $S^{\sharp}$-thickenings coordinate ones. If $(Z, \mathcal{M})$ is log affine (see $1.1)$, then a coordinate thickening always exists, and an arbitrary $(Z, T) \in$ $\mathcal{T}_{S^{\sharp}}$ is pd- $S^{\sharp}$-smooth if and only if it is retract of a coordinate one.

(ii) Suppose $(S, \mathcal{L})$ and $(Z, \mathcal{M})$ are fine, and $i_{Y}:(Z, \mathcal{M}) \hookrightarrow(Y, \mathcal{N})$ is a closed embedding of $\log S^{\sharp}$-schemes with $\left(Y, \mathcal{M}_{Y}\right) \log \operatorname{smooth}$ over $(S, \mathcal{L})$ (see [K1] 3.3). Then its pd- $S^{\sharp}$-envelope is pd- $S^{\sharp}$-smooth.

(iii) For any integral quasi-coherent $\log$ affine $(Z, \mathcal{M})$ over $S^{\sharp}$ there is a universal $i$ as in (i): Take $I=\Gamma\left(Z, \mathcal{O}_{Z}\right), J=\Gamma(Z, \mathcal{M})$, the embedding $i$ is the evident one. The corresponding $\left(P^{\text {univ }}, \mathcal{M}_{P \text { univ }}\right)$ depends on $(Z, \mathcal{M})$ in a functorial way.

Question. Is it true that property of being $\mathrm{pd}-S^{\sharp}$-smooth is étale local?

1.5. Log crystalline site. For $S^{\sharp}$ as in 1.3 and a $\log S^{\sharp}$-scheme $(Z, \mathcal{M})$ which is integral and quasi-coherent, the $\log$ crystalline site $\left((Z, \mathcal{M}) / S^{\sharp}\right)_{\text {crys }}=$ $(Z / S)_{\text {crys }}^{\log }$ is formed by pairs that consist of an étale $Z$-scheme $U / Z$ and a pd- $S^{\sharp}$-thickening $(U, T) \in \mathcal{T}_{S^{\sharp}}$ of $\left(U, \mathcal{M}_{U}\right)$. The coverings are étale ones, i.e., collections of morphisms such that the maps of $T$ 's form an étale covering. The structure sheaf $\mathcal{O}_{Z / S}$ of $(Z / S)_{\text {crys }}^{\log }$ is $\mathcal{O}_{Z / S}(U, T):=\Gamma\left(T, \mathcal{O}_{T}\right)$; let $\mathcal{J}_{Z / S}$ be its canonical pd-ideal, $\mathcal{J}_{Z / S}(U, T):=\Gamma\left(T, \mathcal{J}_{T}\right)$, so $\mathcal{O}_{Z / S} / \mathcal{J}_{Z / S}$ equals $\mathcal{O}_{Z_{\text {ét }}}$. One has a canonical morphism of topoi $u_{Z / S}^{\log }:(Z / S)_{\text {crys }}^{\log \sim} \rightarrow Z_{\text {ét }}$, $u_{Z / S *}^{\log }(\mathcal{F})(U):=\Gamma\left((U / S)_{\text {crys }}^{\log }, \mathcal{F}\right)$.

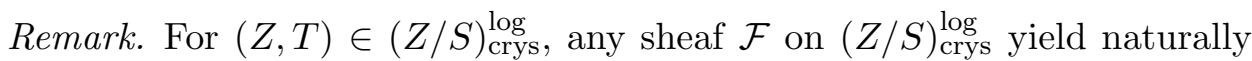
a sheaf $\mathcal{F}_{(Z, T)}$ on $Z_{\text {ét }}=T_{\text {ét }}$ with $\Gamma\left(Z, \mathcal{F}_{(Z, T)}\right)=\mathcal{F}(Z, T)$. The functor $\mathcal{F} \mapsto$ $\mathcal{F}_{(Z, T)}$ is exact, so the evident natural map $u_{Z / S *}^{\log }(\mathcal{F}) \rightarrow \mathcal{F}_{(Z, T)}$ yields one $R u_{Z / S *}^{\log }(\mathcal{F}) \rightarrow \mathcal{F}_{(Z, T)}$.

Proposition. The category $(Z / S)_{\text {crys }}^{\log }$ has non-empty finite inverse limits.

Proof. It suffices to check that it has non-empty finite products and fiber products.

(a) For $\left(U_{i}, T_{i}\right) \in(Z / S)_{\text {crys }}^{\log }, i=1,2$, let us construct their product $(U, T)$. One has $U=U_{1} \times{ }_{Z} U_{2}$. To define $T$, consider the diagonal embedding $i_{Y}:\left(U, \mathcal{M}_{U}\right) \hookrightarrow(Y, \mathcal{N}):=\left(T_{1}, \mathcal{M}_{T_{1}}\right) \times\left(T_{2}, \mathcal{M}_{T_{2}}\right)$. The ideal of $\mathcal{O}_{Y}$ generated by the pullbacks of $\mathcal{J}_{T_{i}}+\mathcal{I} \mathcal{O}_{T_{i}}$ is a pd-ideal, so we have the log pd-scheme $Y^{\sharp}$. Let $\left(U, T^{\prime}\right)$ be the pd- $Y^{\sharp}$-envelope of $i_{Y}$ (it is well defined since $(Y, \mathcal{N})$ is 
integral quasi-coherent). Our $\left(T, \mathcal{M}_{T}\right)$ is an exact closed log pd-subscheme of $\left(T^{\prime}, \mathcal{M}_{T^{\prime}}\right)$ defined as follows. Let $p_{i}$ be the compositions $\left(T^{\prime}, \mathcal{M}_{T^{\prime}}\right) \rightarrow$ $\left(T_{i}, \mathcal{M}_{T_{i}}\right) \rightarrow(S, \mathcal{L})$. Let $\mathcal{J}_{T^{\prime}}^{0}$ be the ideal in $\mathcal{O}_{T^{\prime}}$ generated by local sections $p_{1}^{*}(f)-p_{2}^{*}(f), f \in \mathcal{O}_{S}$, and $\left(p_{1}^{*}(\ell) / p_{2}^{*}(\ell)\right)-1, \ell \in \mathcal{L}$. Here $p_{i}^{*}(\ell)$ are sections of $\mathcal{M}_{T^{\prime}}$ with the same image in $\mathcal{M}_{U}$, so their ratio is a section of $\mathcal{O}_{T^{\prime}}^{\times}$that equals 1 on $U .{ }^{10}$ The ideal $\mathcal{J}_{T^{\prime}}^{0}$ is quasi-coherent since $(S, \mathcal{L})$ is quasi-coherent, and $\mathcal{J}_{T^{\prime}}^{0} \subset \mathcal{J}_{T^{\prime}}$. Now the ideal of $T$ in $\mathcal{O}_{T^{\prime}}$ is the pd-ideal generated by $\mathcal{J}_{T^{\prime}}^{0}$. Since $p_{i}$ coincide on $\left(T, \mathcal{M}_{T}\right)$, our $(U, T)$ is an object of $(Z / S)_{\text {crys }}^{\log }$. We leave it to the reader to check that it is the product of $\left(U_{i}, T_{i}\right)$.

(b) For morphisms $\left(U_{i}, T_{i}\right) \rightarrow(V, Q), i=1,2$, in $(Z / S)_{\text {crys }}^{\log }$, let us construct their fiber product $(W, P)$. One has $W=U_{1} \times_{V} U_{2}$. This is an open subset of $U_{1} \times{ }_{Z} U_{2}$; let $\left(W, P^{\prime}\right)$ be the restriction to $W$ of the product of $\left(U_{i}, T_{i}\right)$ (see (a)). Our $(W, P)$ is an exact closed log pd-subscheme of $\left(W, P^{\prime}\right)$ whose pd-ideal $\mathcal{J}_{P^{\prime}}^{\prime} \subset \mathcal{J}_{P^{\prime}}$ is defined as follows. Let $q_{i}$ be the compositions $\left(P^{\prime}, \mathcal{M}_{P^{\prime}}\right) \rightarrow\left(T_{i}, \mathcal{M}_{T_{i}}\right) \rightarrow\left(Q, \mathcal{M}_{Q}\right)$. We can work étale locally on $V$, so let us assume that $V$ is affine and $\mathcal{M}_{Q}$ has a chart $M \rightarrow \Gamma\left(Q, \mathcal{M}_{Q}\right)$. Now $\mathcal{J}_{P}^{\prime}$ is generated by sections $q_{1}^{*}(g)-q_{2}^{*}(g), g \in \Gamma\left(Q, \mathcal{O}_{Q}\right)$, and $\left(q_{1}^{*}(m) / q_{2}^{*}(m)\right)-1$, $m \in M$.

Let $f:\left(Z^{\prime}, \mathcal{M}^{\prime}\right) / S^{\sharp \sharp} \rightarrow(Z, \mathcal{M}) / S^{\sharp}$ be a map of the above data. A presheaf $\mathcal{F}^{\prime}$ on $\left(Z^{\prime} / S^{\prime}\right)_{\text {crys }}^{\log }$ yields a presheaf $f_{\text {crys } *}\left(\mathcal{F}^{\prime}\right)$ on $(Z / S)_{\text {crys }}^{\log }$ with $f_{\text {crys } *}\left(\mathcal{F}^{\prime}\right)(U, T):=\Gamma\left(\left(\left(U_{Z^{\prime}}, \mathcal{M}_{U_{Z^{\prime}}}^{\prime}\right) / T^{\sharp}\right)_{\text {crys }}, \mathcal{F}^{\prime}\right)$, where $U_{Z^{\prime}}:=U \times_{Z} Z^{\prime}$, $T^{\sharp}:=\left(T, \mathcal{M}_{T}, \mathcal{J}_{T}+\mathcal{I O}_{T}\right)$. If $\mathcal{F}^{\prime}$ is a sheaf, then $f_{\text {crys } *}\left(\mathcal{F}^{\prime}\right)$ is a sheaf. There is an evident map $\mathcal{O}_{Z / S} \rightarrow f_{\text {crys } *}\left(\mathcal{O}_{Z^{\prime} / S^{\prime}}\right)$.

Corollary. $f_{\text {crys } *}$ defines a morphism of ringed topoi $f_{\text {crys }}:\left(Z^{\prime} / S^{\prime}\right)_{\text {crys }}^{\log \tilde{s}} \rightarrow$ $(Z / S)_{\text {crys }}^{\log \tilde{}}$.

Proof. For $\left(U^{\prime}, T^{\prime}\right) \in\left(Z^{\prime} / S^{\prime}\right)_{\text {crys }}^{\log }$ let $C_{f}=C_{f}\left(U^{\prime}, T^{\prime}\right)$ be the category of pairs $((U, T), g)$ where $(U, T) \in(Z / S)_{\text {crys }}^{\log }, g:\left(U^{\prime}, T^{\prime}\right) \rightarrow(U, T)$ is a map of pd- $S^{\sharp}-$ thickenings compatible with $f$. The proposition implies that $C_{f}$ has finite inverse limits, so $C_{f}^{\circ}$ is directed. For a presheaf $\mathcal{F}$ on $(Z / S)_{\text {crys }}^{\log }$, its pullback $f_{\text {crys }}^{-1}(\mathcal{F})$ assigns to $\left(U^{\prime}, T^{\prime}\right)$ the colimit of the functor $((U, T), g) \mapsto \mathcal{F}(U, T)$ on $C_{f}^{\circ} .{ }^{11}$ If $\mathcal{F}$ is a sheaf, then $f_{\text {crys }}^{*}(\mathcal{F})$ is the sheaf associated with $f_{\text {crys }}^{-1}(\mathcal{F})$. Since $C_{f}^{\circ}$ is directed, $f_{\text {crys }}^{-1}$ commutes with finite inverse limits, so same is true for $f_{\text {crys }}^{*}$. We are done.

\footnotetext{
${ }^{10}$ Since $\left(U, \mathcal{M}_{U}\right) \hookrightarrow\left(T^{\prime}, \mathcal{M}_{T^{\prime}}\right)$ is an exact embedding with nil ideal $\mathcal{J}_{T^{\prime}}, \mathcal{M}_{U}$ is the quotient of $\mathcal{M}_{T^{\prime}}$ modulo the action of $1+\mathcal{J}_{T^{\prime}} \subset \mathcal{O}_{T^{\prime}}^{\times}$, and this action is free since $\mathcal{M}_{T^{\prime}}$ is integral.

${ }^{11} C_{f}^{\circ}$ contains a small cofinal subcategory, so the colimit is well defined.
} 


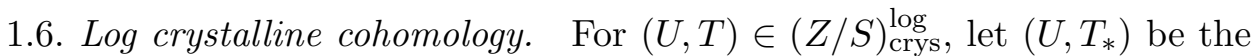
restriction to $U \subset U \times{ }_{Z} \ldots \times_{Z} U$ of the standard simplicial object of $(Z / S)_{\text {crys }}^{\text {log }}$ with terms $\left(U, T_{a}\right):=(U, T)^{[0, a]}$ (the product is computed in $(Z / S)_{\text {crys }}^{\log }$, see Proposition in 1.5). The construction is natural and compatible with étale localization, hence any sheaf $\mathcal{F}$ on $(Z / S)_{\text {crys }}^{\log }$ yields a cosimplicial sheaf $(U, T) \mapsto \mathcal{F}\left(U, T_{*}\right)$. If $\mathcal{F}$ is a sheaf of abelian groups, then let $\mathcal{C} \mathcal{F}$ be the normalized complex of this cosimplicial sheaf. The functor $\mathcal{C}$ is exact. There is an evident projection $\alpha: \mathcal{C} \cdot \mathcal{F} \rightarrow \mathcal{F}$.

The embedding $H^{0} \mathcal{C} \cdot \mathcal{F} \hookrightarrow \mathcal{F}$ yields an isomorphism $u_{Z / S *}^{\log }\left(H^{0} \mathcal{C} \cdot \mathcal{F}\right) \stackrel{\sim}{\rightarrow}$ $u_{Z / S *}^{\log }(\mathcal{F})$, so we have a natural map $\beta: u_{Z / S *}^{\log }(\mathcal{F}) \rightarrow u_{Z / S *}^{\log }(\mathcal{C} \cdot \mathcal{F})$ right inverse to $u_{Z / S *}^{\log }(\alpha)$.

Suppose we have $(Z, T) \in(Z / S)_{\text {crys }}^{\log }$. Due to exactness of $\mathcal{C}$, the natural $\operatorname{map} u_{Z / S *}^{\log }(\mathcal{C} \cdot \mathcal{F}) \rightarrow \mathcal{C} \cdot \mathcal{F}_{(Z, T)}$ yields one $R\left(u_{Z / S *}^{\log } \mathcal{C} \cdot\right)(\mathcal{F}) \rightarrow \mathcal{C} \cdot \mathcal{F}_{(Z, T)}$.

Proposition. (i) One has $R\left(u_{Z / S *}^{\log } \mathcal{C} \cdot\right)(\mathcal{F}) \stackrel{\sim}{\rightarrow} R u_{Z / S *}^{\log }(\mathcal{F})$.

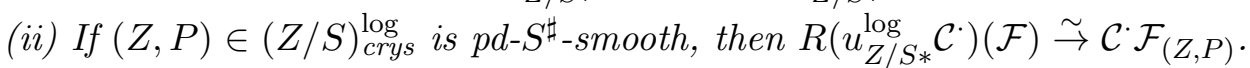
Thus

$$
R u_{Z / S *}^{\log }(\mathcal{F}) \stackrel{\sim}{\rightarrow} \mathcal{C} \cdot \mathcal{F}_{(Z, P)}
$$

Proof. Deriving $\alpha$ and $\beta$, we get $R\left(u_{Z / S *}^{\log } \mathcal{C}^{\cdot}\right)(\mathcal{F}) \leftrightarrows R u_{Z / S *}^{\log }(\mathcal{F})$ whose composition in one direction is identity; we want to check they are mutually inverse. The problem is local, so we can assume that there is $P$ as in (ii) and $Z$ is affine. Since $(Z, P)$ is pd- $S^{\sharp}$-smooth, one has $u_{Z / S *}^{\log }(\mathcal{F}) \stackrel{\sim}{\rightarrow} H^{0} \mathcal{C} \cdot \mathcal{F}_{(Z, P)}$. To prove (i), (ii), it suffices then to find for every $\mathcal{F}$ an embedding $\mathcal{F} \hookrightarrow \tilde{\mathcal{F}}$ such that $H^{\neq 0} \mathcal{C} \cdot \tilde{\mathcal{F}}_{(Z, P)}=0$.

For $(Z, T) \in(Z / S)_{\text {crys }}^{\log }$ consider the simplicial object $\left(Z, P_{*}\right) \times(Z, T)$ augmented over $(Z, T)$. The augmentation admits an inverse up to homotopy: indeed, any map $(Z, T) \rightarrow(Z, P)$ yields one in the usual way, and such a map exists since $(Z, P)$ is pd- $S^{\sharp}$-smooth. So the sheaf $\left(U, T^{\prime}\right) \mapsto \tilde{\mathcal{F}}\left(U, T^{\prime}\right):=$ $\mathcal{F}\left(\left(U, T^{\prime}\right) \times(Z, T)\right)$ satisfies $H^{\neq 0} \mathcal{C} \cdot \tilde{\mathcal{F}}_{(Z, P)}=0$. If $(Z, T)$ is pd- $S^{\sharp}$-smooth (say, $(Z, T)=(Z, P))$, then the evident map $\nu: \mathcal{F} \rightarrow \tilde{\mathcal{F}}$ is injective; ${ }^{12}$ q.e.d.

Remark. (i) Suppose $(Z, P)$ is a coordinate thickening as in Remark (i) in 1.4. The $\mathbb{G}_{a}^{I} \times \mathbb{G}_{m}^{J}$-action on $\mathbb{A}_{(S, \mathcal{L})}^{I} \times(S, \mathcal{L}) \mathbb{A}_{(S, \mathcal{L})}^{(J)}(\text { see } 1.1)^{13}$ yields, by universality,

\footnotetext{
${ }^{12}$ Indeed, for $U$ affine one can find a map $\left(U, T^{\prime}\right) \rightarrow(Z, T)$, which yields a left inverse to $\nu$.
} 
a $\mathbb{G}_{a}^{\sharp I} \times \mathbb{G}_{m}^{\sharp J}$-action on $(Z, P)$ (see 1.2). Then $(Z, P)$ is a $\mathbb{G}_{a}^{\sharp I} \times \mathbb{G}_{m}^{\sharp J}$-torsor on $(Z / S)_{\text {crys }}^{\log \text {. Thus }}\left(Z, P_{a}\right)=\left(\mathbb{G}_{a}^{\sharp I} \times \mathbb{G}_{m}^{\sharp J}\right)^{a} \times(Z, P)$ and $\left(Z, P_{*}\right)$ is the "universal simplicial $\mathbb{G}_{a}^{\sharp I} \times \mathbb{G}_{m}^{\sharp J}$-quotient" for the $\mathbb{G}_{a}^{\sharp I} \times \mathbb{G}_{m}^{\sharp J}$-action on $(Z, P)$.

(ii) If $(Z, P)$ is pd- $S^{\sharp}$-smooth, then it is a covering of $(Z / S)_{\text {crys }}^{\log }$.

(iii) If no pd- $S^{\sharp}$-smooth $(Z, P)$ is available, one can compute $R u_{Z / S *}^{\log }(\mathcal{F})$ by either of the next procedures:

- By Remark (i) in 1.4 and Proposition in 1.5, any étale hypercovering $p: U . \rightarrow Z$ with $\log$ affine $\left(U_{0}, \mathcal{M}_{U_{0}}\right)$ has a pd- $S^{\sharp}$-smooth thickening $(U ., P$.$) ,$ so $R u_{Z / S *}^{\log }(\mathcal{F}) \stackrel{\sim}{\rightarrow} R p_{*} \mathcal{C} \cdot \mathcal{F}_{(U ., P .)}$.

- Replace $Z_{\text {ét }}$ by an equivalent topology $Z_{\text {ét }}$ formed by those étale $U / Z$ that $\left(U, \mathcal{M}_{U}\right)$ is $\log$ affine. Then $U \mapsto \Gamma\left(U, \mathcal{C} \cdot \mathcal{F}_{\left(U, P^{\text {univ }}\right)}\right)$ (see Remark (iii) in 1.4) is a presheaf on $Z_{\text {ét' }}$; its sheafification equals $R u_{Z / S *}^{\log }(\mathcal{F})$.

- Consider for each $U \in X_{\text {ét }}$ the category $\mathcal{S}(U)$ of its pd- $S^{\sharp}$-smooth thickenings. Then ${ }^{14}$ the presheaf $U \mapsto \operatorname{holim}_{(U, P) \in \mathcal{S}(U)} R \Gamma\left(U, \mathcal{C} \cdot \mathcal{F}_{(U, P)}\right)$ represents $R u_{Z / S *}^{\log }(\mathcal{F})$.

1.7. Log $\mathcal{O}$-crystals and connections. For $(U, T) \in(Z / S)_{\text {crys }}^{\log }$ its de Rham pd-algebra $\Omega_{(U, T) / S}$ is the quotient of the log de Rham dg algebra $\Omega_{\left(T, \mathcal{M}_{T}\right) /(S, \mathcal{L})}$ (see $[\mathrm{K} 1] 1.7,1.9)$ modulo the relations $d\left(u^{[n]}\right)=u^{[n-1]} d u, u \in \mathcal{J}_{T}$. This is a sheaf of commutative dg $\mathcal{O}_{S}$-algebras on $T_{\text {ét }}=U_{\text {ét }}$ whose terms are quasi-coherent $\mathcal{O}_{T}$-modules (since $\left(T, \mathcal{M}_{T}\right)$ and $(S, \mathcal{L})$ are quasi-coherent). It carries the Hodge pd-filtration $F^{m}, F^{m} \Omega_{(Z, T) / S}^{a}:=\mathcal{J}_{T}^{[m-a]} \Omega_{(Z, T) / S}^{a}$; one has $F^{m} \cdot F^{\ell} \subset F^{m+\ell}$. We get a sheaf $\Omega_{Z / S}$ of filtered commutative dg algebras on $(Z / S)_{\text {crys }}^{\log }, \Omega_{Z / S}(U, T):=\Gamma\left(T, \Omega_{(U, T) / S}\right)$.

Exercises. (i) If $(U, T)$ is the pd-envelope of $(U, \mathcal{M}) \hookrightarrow(Y, \mathcal{N})$ as in (1.3.1), then the composition $r^{*} \Omega_{(Y, \mathcal{N}) /(S, \mathcal{L})}^{1} \stackrel{r^{*}}{\rightarrow} \Omega_{\left(T, \mathcal{M}_{T}\right) /(S, \mathcal{L})}^{1} \rightarrow \Omega_{(U, T) / S}^{1}$ is an isomorphism.

(ii) If $(U, T)$ is a pd-smooth thickening and $U$ is affine, then $\Omega_{Z / S}^{1}(U, T)$ is a projective $\Gamma\left(T, \mathcal{O}_{T}\right)$-module. ${ }^{15}$

Here is another description of $\Omega_{Z / S}$. For $(U, T) \in(Z / S)_{\text {crys }}^{\log }$ let $\left(U, T_{*}\right)$ be the simplicial object defined in 1.6. Let $\mathcal{V}_{T_{1}} \subset \mathcal{O}_{T_{1}}$ be the ideal of the

\footnotetext{
${ }^{13}$ An element $\left(\left(h_{i}\right),\left(g_{j}\right)\right)_{i \in I, j \in J} \in \mathbb{G}_{a}^{I} \times \mathbb{G}_{m}^{J}$ acts as $t_{i} \mapsto t_{i}-h_{i}, t_{j} \mapsto g_{i}^{-1} t_{j}$.

${ }^{14}$ To define holim, one should take care of the usual set-theoretic difficulties.

${ }^{15}$ Our assertion is local, so we can assume that $(Z, \mathcal{M})$ is log affine; by Remark (i) in 1.4, we can assume that $(Z, T)$ is a coordinate thickening; now the assertion follows from (i).
} 
subscheme $T=T_{0}$ of $T_{1}, \mathcal{V}_{T_{1}}^{[2]} \subset \mathcal{O}_{T_{1}}$ be its divided powers square. Let $T_{*}^{b}$ be the closed exact simplicial log subscheme of $T_{*}$ whose cosimplicial ideal in $\mathcal{O}_{T_{*}}$ is generated by $\mathcal{V}_{T_{1}}^{[2]}$. The normalized complex $\mathrm{N} \cdot\left(\mathcal{O}_{T_{*}^{\mathrm{b}}}\right)$ equipped with the Alexander-Whitney product is a sheaf of dg algebras on $U_{\text {ét }}$ that depends functorially on $(U, T)$. Thus we have a sheaf $\Omega_{Z / S}^{b}$ of dg algebras on $(Z / S)_{\text {crys }}^{\log }, \Omega_{Z / S}^{b \cdot}(U, T):=\Gamma\left(U, \mathrm{~N}^{\cdot}\left(\mathcal{O}_{T_{*}^{b}}\right)\right)$.

Proposition. One has a natural isomorphism of dg algebras $\phi^{\cdot}: \Omega_{Z / S} \stackrel{\sim}{\rightarrow}$ $\Omega_{Z / S}^{b \cdot}$

Proof. We will construct a natural isomorphism $\varphi^{\cdot}: \Omega_{(U, T) / S} \stackrel{\sim}{\rightarrow} \mathrm{N}^{\cdot}\left(\mathcal{O}_{T_{*}^{b}}\right)$ of sheaves of dg algebras on $U_{\text {ét. }}$. It satisfies the properties $\phi^{0}=\mathrm{id}_{\mathcal{O}_{T}}$ and $\phi^{1}(d \log (m))=\left(p_{1}^{*}(m) / p_{0}^{*}(m)\right)-1 \in \mathcal{V}_{T_{1}} / \mathcal{V}_{T_{1}}^{[2]}=\mathrm{N}^{1}\left(\mathcal{O}_{T_{*}^{b}}\right)$ for $m \in \mathcal{M}_{T}$ (here $p_{i}: T_{1}^{b} \rightarrow T$ are the projections) that determine $\varphi$ uniquely. ${ }^{16}$

(a) Recall that, by Dold-Puppe, the normalization functor $\mathrm{N}^{*}$ is an equivalence between the category of cosimplicial abelian groups and the category of complexes vanishing in negative degrees; let $\mathrm{K}^{*}$ be the inverse equivalence. Both categories carry the usual symmetric tensor products $\otimes$. The functors $\mathrm{N}^{*}$ and $\mathrm{K}^{*}$ transform algebras to algebras (using the Alexander-Whitney and shuffle products respectively); both send associative algebras to associative ones, and $\mathrm{K}^{*}$ transforms commutative algebras to commutative ones. If $C^{\cdot}$ is a dg algebra, then the identification of complexes $\mathrm{N}^{*} \mathrm{~K}^{*}\left(C^{\cdot}\right) \stackrel{\sim}{\rightarrow} C^{\cdot}$ is compatible with the products.

Some explicit formulas: Below $\partial_{i}:[0, n-1] \rightarrow[0, n], \sigma_{i}:[0, n] \rightarrow[0, n-1]$ are the standard face and degeneration maps, $\sigma_{i} \partial_{i}=\sigma_{i} \partial_{i+1}=\mathrm{id}_{[0, n-1]}$. Let $A^{*}$ be a cosimplicial abelian group and $C$ be a complex that correspond one to another by $\mathrm{N}^{*}$ and $K^{*}$. For a monotone map $e:[0, m] \rightarrow[0, n]$ we write the cosimplicial structure map $e=e_{A}: A^{m} \rightarrow A^{n}$ also as $a \mapsto^{e} a$. One has $C^{n}=\cap \operatorname{Ker} \sigma_{i} \subset A^{n}$, and $d=\left.\Sigma(-1)^{i} \partial_{i}\right|_{C}$. For $m \in[0, n]$ let $E(m, n)$ be the set of increasing injections $e:[0, m] \hookrightarrow[0, n]$ such that $e(0)=0 .{ }^{17}$ Let ${ }^{e} C^{m} \subset A^{n}$ be the image of $C^{m} \subset A^{m}$ by the (injective) map $e_{A}$. One has a Dold-Puppe direct sum decomposition

$$
A^{n}=\oplus_{m \leq n} \oplus_{e \in E(m, n)}{ }^{e} C^{m} .
$$

\footnotetext{
${ }^{16}$ Since $\Omega_{(U, T) / S}$ is generated, as a dg algebra, by $\Omega_{(U, T) / S}^{0}$ and $d \log \left(\mathcal{M}_{T}\right) \subset$ $\Omega_{(U, T) / S}^{1}$.

${ }^{17}$ The map $e \mapsto e([1, m])$ identifies $E(m, n)$ with the set of all order $m$ subsets of $[1, n]$.
} 
For a monotone $g:[0, n] \rightarrow[0, \ell]$ the components of the map $g_{A}: A^{n} \rightarrow A^{\ell}$ are as follows: For ${ }^{e} c \in{ }^{e} C^{m}$ one has $g_{A}\left({ }^{e} c\right)=0$ if $g e:[0, m] \rightarrow[0, \ell]$ is not injective, and $g_{A}\left({ }^{e} c\right)={ }^{g e} c$ if $g e \in E(m, \ell)$. Otherwise we have $[g e] \in E(m+$ $1, \ell)$ such that $[g e] \partial_{0}=g e$, and $g_{A}\left({ }^{e} c\right)={ }^{[g e]}(d c)-\Sigma_{1 \leq i \leq m+1}(-1)^{i}{ }^{[g e] \partial_{i}} c$. Here $\partial_{i}:[0, m] \rightarrow[0, m+1]$ are the usual face maps.

If $C$ is a dg algebra, then the corresponding shuffle product on $A^{*}$ looks as follows. For $e_{1} \in E\left(m_{1}, n\right), e_{2} \in E\left(m_{2}, n\right), c_{1} \in C^{m_{1}}, c_{2} \in C^{m_{2}}$ the shuffle product ${ }^{e_{1}} c_{1}{ }^{e_{2}} c_{2}$ vanishes if $e\left(\left[1, m_{1}\right]\right) e_{2}\left(\left[1, m_{2}\right]\right) \neq \emptyset$; otherwise it equals $\pm^{e}\left(c_{1} c_{2}\right)$ where $e \in E\left(m_{1}+m_{2}, n\right)$ has image $\{0\} \cup e_{1}\left(\left[1, m_{1}\right]\right) \cup e_{2}\left(\left[1, m_{2}\right]\right)$ and \pm is the sign of the permutation $\sigma$ of $\left[1, m+m^{\prime}\right]$ such that $\sigma(a)$ equals $e^{-1} e_{1}(a)$ if $a \leq m_{1}$, and $e^{-1} e_{2}(a-m)$ if $a>m_{1}$. Notice that the product on each ${ }^{e} C^{m}$ vanishes if $m \neq 0$.

Suppose $C^{\cdot}$ is a strictly ${ }^{18}$ commutative dg algebra, so the shuffle product on $A^{*}$ is commutative. Let $V^{n}$ be the kernel of the degeneration map $A^{n} \rightarrow$ $A^{0}$. Then $V^{*}$ is a cosimplicial ideal in $A^{*}$. It carries a unique pd structure such that the cosimplicial structure maps are pd-morphisms and $c^{[k]}=0$ for $c \in C^{m} \subset A^{m}, k>1, m>0 .{ }^{19}$ More generally, suppose we have a pd-ideal $J \subset C^{0}=A^{0}$. Then the pd structures on $J$ and $V^{*}$ are compatible (i.e., extend to the pd structure on the cosimplicial ideal $J_{A}^{*}$ they generate that is compatible with the cosimplicial structure maps) if and only if for every $f \in J, n>0$ one has $d\left(f^{[n]}\right)=f^{[n-1]} d(f) .{ }^{20}$

(b) Set $\Omega:=\Omega_{(U, T) / S}$, and consider the cosimplicial commutative algebra $A^{*}:=\mathrm{K}^{*}(\Omega)$. By (a), the pd structure on $\mathcal{J}_{T} \subset \mathcal{O}_{T}=A^{0}$ yields a pd structure on the corresponding cosimplicial ideal $J_{A}^{*}$; notice that $A^{n} / J_{A}^{n}=$ $\mathcal{O}_{T} / \mathcal{J}_{T}=\mathcal{O}_{U}$. The $\log$ structure $\mathcal{M}_{T}$ extends naturally to a $\log$ structure on the simplicial scheme Spec $A^{*}$ : We define $\mathcal{M}_{\text {Spec }} A^{n}$ as the pullback of $\mathcal{M}_{T}$ by either of the $n+1$ simplicial structure projections $p_{i}=\operatorname{Spec}\left(\delta_{i A}\right)$ :

${ }^{18}$ Which means that $c^{2}=0$ for $c$ of odd degree.

${ }^{19}$ One has $V^{n}=\oplus_{m>0}{ }^{e} C^{m}$ as in (1.7.1). For $v \in V^{n}$ let $v_{m, e} \in{ }^{e} C^{m}$ be the components of $v$. If we have our pd-structure, then $v_{m, e}^{[>1]}=0$ implies that $v^{[k]}=$ $\sum_{P} \prod_{(m, e) \in P} v_{(m, e)}$, where $P$ runs the set of all $k$ element subsets of $\sqcup_{m>0} E(m, n)$. Now take this formula as the definition of divided powers on $V^{n}$. The axioms of pd-structure are clear. To show that $g_{A}: A^{n} \rightarrow A^{\ell}$ are pd-morphisms, it suffices to check that $\left(g_{A}\left(v_{m, e}\right)\right)^{[k]}=0$ for $k>1$. This is immediate for $m>1$ and follows from strict commutativity for $m=1$.

${ }^{20}$ Notice that $J_{A}^{n}={ }^{\delta_{0}} J \oplus V^{n}$, where ${ }^{\delta_{0}} J \subset{ }^{\delta_{0}} C^{0},\left\{\delta_{0}\right\}=E(0, n)$. So we set $\left({ }^{\delta_{0}} f+\right.$ $v)^{[k]}:=\Sigma_{a}{ }^{\delta_{0}} f^{[a]} v^{[k-a]}$. One easily checks the pd structure axioms. The compatibility with cosimplicial structure is enough to check for the map $\partial_{0 A}: J \rightarrow J_{A}^{1}$. Then $\partial_{0 A}(f)^{[n]}=\left({ }^{\delta_{0}} f+d f\right)^{[n]}={ }^{\delta_{0}} f^{[n]}+f^{[n-1]} d f$ and $\partial_{0 A}\left(f^{[n]}\right)={ }^{\delta_{0}} f^{[n]}+d\left(f^{[n]}\right)$, q.e.d. 
$\operatorname{Spec} A^{n} \rightarrow \operatorname{Spec} A^{0}=T$, where $\delta_{i}:[0,0] \rightarrow[0, n]$ is $\delta_{i}(0)=i$. The pullbacks are identified as $p_{i}^{*}(m)=\left(1-\delta_{i, j A}(d \log (m))\right) p_{j}^{*}(m)$ for $i<j, m \in \mathcal{M}_{T}$, where $\delta_{i, j}:[0,1] \rightarrow[0, n]$ is $\delta_{i, j}(0)=i, \delta_{i, j}(1)=j$, and $d \log (m) \in \Omega^{1} \subset A^{1}$. The log and pd structures make $\left(U, \operatorname{Spec} A^{*}\right)$ a simplicial object of $(Z / S)_{\text {crys }}^{\log }$. By the definition of $T_{*}$, there is a unique map $\left(U, \operatorname{Spec} A^{*}\right) \rightarrow\left(U, T_{*}\right)$ in $(Z / S)_{\text {crys }}^{\log }$ that equals $i_{T}$ in degree 0 . It evidently takes image in $\left(U, T_{*}^{b}\right)$.

We have defined a morphism of cosimplicial algebras $\mathcal{O}_{T_{*}^{b}} \rightarrow A^{*}$, hence a morphism of dg algebras $\psi^{*}: \mathrm{N}^{\cdot}\left(\mathcal{O}_{T_{*}^{b}}\right) \rightarrow \mathrm{N}^{\cdot}\left(A^{*}\right)=\Omega$. It remains to show that $\psi^{\prime}$ is an isomorphism: the promised identification $\varphi^{\prime}$ is its inverse.

(c) Below $\mathrm{N}^{\cdot}:=\mathrm{N}^{\cdot}\left(\mathcal{O}_{T_{*}^{b}}\right)$. Let us first check that $\psi^{1}: \mathrm{N}^{1} \rightarrow \Omega^{1}$ is an isomorphism. We have $T \hookrightarrow T_{1}^{b}$ and the two retractions $p_{0}, p_{1}: T_{1}^{b} \rightarrow T$. Our $\mathrm{N}^{1}$ is the ideal of $T$ in $\mathcal{O}_{T_{1}^{b}}$; it has square zero; $p_{0}$ yields a splitting $\mathcal{O}_{T} \oplus$ $\mathrm{N}^{1} \stackrel{\sim}{\rightarrow} \mathcal{O}_{T_{1}^{b}}$ and an identification of $\log$ structures $p_{0}^{*}\left(\mathcal{M}_{T}\right) \stackrel{\sim}{\rightarrow} \mathcal{M}_{T_{1}^{b}}$. The other retraction $p_{1}$ amounts then to an $\mathcal{O}_{T}$-linear map $\phi^{1}: \Omega_{\left(T, \mathcal{M}_{T}\right) /(S, \mathcal{L})}^{1} \rightarrow$ $\mathrm{N}^{1}$ such that $\phi^{1}(d(f))=p_{1}^{*}(f)-p_{0}^{*}(f), \phi^{1}(d \log (m))=p_{1}^{*}(m) / p_{0}^{*}(m)-1$. Since $p_{i}$ are pd-morphisms and divided powers of degree $>1$ vanish on $\mathrm{N}^{1}$, $\phi^{1}$ factors through $\Omega^{1}$ (cf. the computation in the last footnote in (a)). Since the images of $\mathcal{O}_{T}$ and $\mathcal{M}_{T}$ by $p_{0}^{*}$ and $p_{1}^{*}$ generate $\mathcal{O}_{T_{1}^{b}}, \phi^{1}$ is surjective. Since $\psi^{1} \phi^{1}=\mathrm{id}_{\Omega^{1}}$ by the construction of $\psi^{1}, \phi^{1}$ is inverse to $\psi^{1}$.

(d) For $n \geq 2$, let $\sigma_{i}: \mathcal{O}_{T_{n}^{b}} \rightarrow \mathcal{O}_{T_{n-1}^{b}}, i \in[0, n-1]$, be the standard degeneration maps, $\sigma_{i} \partial_{i}=\sigma_{i} \partial_{i+1}=\operatorname{id}_{[0, n-1]}$. Then the kernel $\mathcal{V}_{T_{n}^{b}}$ of the degeneration map $\mathcal{O}_{T_{n}^{b}} \rightarrow \mathcal{O}_{T}$ equals $\Sigma_{i} \operatorname{Ker}\left(\sigma_{i}\right), \mathrm{N}^{n}=\cap_{i} \operatorname{Ker}\left(\sigma_{i}\right)$, and $\operatorname{Ker}\left(\sigma_{i}\right)$ equals the ideal in $\mathcal{O}_{T_{n}^{b}}$ generated by $\delta_{i, i+1}\left(\mathrm{~N}^{1}\right)$. Thus $\operatorname{Ker}\left(\sigma_{i}\right)^{[2]}=\mathcal{V}_{T_{n}^{b}} \mathrm{~N}^{n}=$ $\left(\mathrm{N}^{n}\right)^{[2]}=0$.

For $c \in \mathrm{N}^{1}$ one has $c \cup c=0$ (here $\cup$ is the Alexander-Whitney product). ${ }^{21}$ Thus the subalgebra of $\mathrm{N}^{\cdot}$ generated by $\mathrm{N} \leq 1$ is strictly commutative. This subalgebra is closed under differential, since $\mathrm{N}^{1}$ is generated, as an $\mathcal{O}_{T^{-}}$ module, by cycles. ${ }^{22}$ By the universality property of $\Omega$ and (c), there is a unique map of dg algebras $\phi^{\prime}: \Omega \rightarrow \mathrm{N}^{\prime}$ such that $\psi^{\circ} \phi^{\prime}=\mathrm{id}_{\Omega}$.

It remains to prove that $\phi$ is surjective, or, equivalently, that the image $A^{*}$ of $K^{*}\left(\phi^{*}\right)$ equals $\mathcal{O}_{T_{*}^{b}}$. Since $\mathcal{O}_{T_{*}^{b}}$ is generated, as cosimplicial algebra, by $\mathcal{O}_{T_{1}^{b}}$, it suffices to show that $A^{*}$ is a subalgebra of $\mathcal{O}_{T_{*}^{b}}$. Notice that $A^{n}, n>0$, is generated, as an abelian group, by elements $\delta_{0}(f)$, $f \in \mathcal{O}_{T}$, and $e_{A}\left(\nu_{1} \cup \ldots \cup \nu_{m}\right), \nu_{i} \in \Omega^{1}, e \in E(m, n), 0<m \leq n$. One

${ }^{21}$ Indeed, $c \cup c:=\partial_{2}(c) \partial_{0}(c)=\left(\partial_{2}(c)+\partial_{0}(c)\right)^{[2]}=\left(\partial_{1}(c)+d(c)\right)^{[2]}=0$, since $\partial_{i}(c)^{[2]}=\partial_{1}(c) d(c)=d(c)^{[2]}=0$.

${ }^{22}$ Namely, $d(f)$ and $\phi^{1}(d \log (m))$, where $f \in \mathcal{O}_{T}, m \in \mathcal{M}_{T}$, see $(\mathrm{c})$. 
has $\delta_{0}(f) e_{A}\left(\nu_{1} \cup \ldots \cup \nu_{m}\right)=e_{A}\left(f \nu_{1} \cup \ldots \cup \nu_{m}\right)$. Since $\delta_{0, j}(\nu) \delta_{0, j}\left(\nu^{\prime}\right) \in$ $\delta_{0, j}\left(\left(\mathrm{~N}^{1}\right)^{2}\right)=0$, one has $\nu_{1} \cup \ldots \cup \nu_{m}:=\delta_{0,1}\left(\nu_{1}\right) \delta_{1,2}\left(\nu_{2}\right) \ldots \delta_{m-1, m}\left(\nu_{m}\right)=$ $\delta_{0,1}\left(\nu_{1}\right) \delta_{0,2}\left(\nu_{2}\right) \ldots \delta_{0, m}\left(\nu_{m}\right) .{ }^{23}$ Thus $e_{A}\left(\nu_{1} \cup \ldots \cup \nu_{m}\right) e_{A^{\prime}}\left(\nu_{1}^{\prime} \cup \ldots \cup \nu_{m^{\prime}}^{\prime}\right)=$ $\delta_{0, e(1)}\left(\nu_{1}\right) \ldots \delta_{0, e(m)}\left(\nu_{m}\right) \delta_{0, e^{\prime}(1)}\left(\nu_{1}^{\prime}\right) \ldots \delta_{0, e^{\prime}\left(m^{\prime}\right)}\left(\nu_{m^{\prime}}^{\prime}\right)$ vanishes if $e([1, m]) \cap$ $e^{\prime}\left(\left[1, m^{\prime}\right]\right) \neq \emptyset$ and otherwise equals $\pm e_{A}^{\prime \prime}\left(\nu_{1} \cup \ldots \cup \nu_{m} \cup \nu_{1}^{\prime} \cup \ldots \cup \nu_{m^{\prime}}^{\prime}\right)$, where $\left.e^{\prime \prime} \in E\left(m+m^{\prime}, n\right), e^{\prime \prime}\left(\left[1, m+m^{\prime}\right]\right)=e([1, m]) \cup e^{\prime}\left[1, m^{\prime}\right]\right)$. We are done.

A sheaf $\mathcal{F}$ of $\mathcal{O}_{Z / S^{-}}$-modules on $(Z / S)_{\text {crys }}^{\log }$ is said to be $\mathcal{O}_{Z / S^{-}}$crystal if for every morphism $\phi:\left(U^{\prime}, T^{\prime}\right) \rightarrow(U, T)$ in $(Z / S)_{\text {crys }}^{\log }$ the pullback map $\phi^{-1} \mathcal{F}_{(U, T)} \rightarrow \mathcal{F}_{\left(U^{\prime}, T^{\prime}\right)}$ yields an isomorphism of $\mathcal{O}_{T^{\prime}}$-modules $\phi^{*} \mathcal{F}_{(U, T)} \stackrel{\sim}{\rightarrow}$ $\mathcal{F}_{\left(U^{\prime}, T^{\prime}\right)}$. For such an $\mathcal{F}$, let $\Omega_{Z / S} \mathcal{F}$ be the normalization of the cosimplicial sheaf $(U, T) \mapsto \mathcal{F}\left(U, T_{*}^{b}\right)$. By Proposition, $\Omega_{Z / S} \mathcal{F}$ is a $\operatorname{dg} \Omega_{Z / S}$-module and $\Omega_{Z / S}^{i} \mathcal{F}=\Omega_{Z / S}^{i} \otimes_{\mathcal{O}_{Z / S}} \mathcal{F}$. So $\Omega_{Z / S} \mathcal{F}$ is the de Rham complex for the flat connection $\nabla:=d^{0}: \mathcal{F} \rightarrow \Omega_{Z / S}^{1} \otimes_{\mathcal{O}_{Z / S}} \mathcal{F}$.

Theorem. Suppose $(Z, P)$ is a $p d-S^{\sharp}$-smooth thickening. Then the connection $\nabla_{(Z, P)}: \mathcal{F}_{(Z, P)} \rightarrow \Omega_{(Z, P) / S}^{1} \otimes_{\mathcal{O}_{P}} \mathcal{F}_{(Z, P)}$ is quasi-nilpotent (see [K1] 6.2). The functor $\mathcal{F} \mapsto\left(\mathcal{F}_{(Z, P)}, \nabla_{(Z, P)}\right)$ is an equivalence between the category of $\mathcal{O}_{Z / S}$-crystals and that of $\mathcal{O}_{P}$-modules equipped with an integrable quasinilpotent connection.

Proof. This is theorem 6.2 from [K1] (it is stated in loc. cit. under the assumption that $\mathcal{M}$ is fine; the proof works in our setting as well).

1.8. Comparison with the de Rham cohomology. Below we call $R u_{Z / S *}^{\log }\left(\mathcal{O}_{Z / S}\right)$ and $R \Gamma\left((Z / S)_{\text {crys }}^{\log }, \mathcal{O}_{Z / S}\right)=R \Gamma\left(Z_{\text {ét }}, R u_{Z / S *}^{\log }\left(\mathcal{O}_{Z / S}\right)\right)$ simply the log crystalline complexes. These are $\mathrm{E}_{\infty}$ algebras. ${ }^{24}$ Suppose $\mathcal{F}$ is an $\mathcal{O}_{Z / S}$-crystal, so $R u_{Z / S *}^{\log }(\mathcal{F})$ is a $R u_{Z / S *}^{\log }\left(\mathcal{O}_{Z / S}\right)$-module, $R \Gamma\left((Z / S)_{\text {crys }}^{\log }, \mathcal{F}\right)$ is an $R \Gamma\left((Z / S)_{\text {crys }}^{\log }\right.$, $\mathcal{O}_{Z / S}$ )-module.

Theorem. (i) The evident projection $\Omega_{Z / S} \rightarrow \mathcal{O}_{Z / S}$ yields a quasi-isomorphism $R u_{Z / S *}^{\log }\left(\Omega_{Z / S} \mathcal{F}\right) \stackrel{\sim}{\rightarrow} R u_{Z / S *}^{\log }(\mathcal{F})$.

\footnotetext{
${ }^{23}$ Indeed, by induction by $m$, one has $\nu_{1} \cup \ldots \cup \nu_{m}=\delta_{0,1}\left(\nu_{1}\right) \ldots \delta_{0, m-1}\left(\nu_{m-1}\right) \times$ $\delta_{m-1, m}\left(\nu_{m}\right)$. Since $\delta_{m-1, m}\left(\nu_{m}\right)-\delta_{0, m}\left(\nu_{m}\right)=\delta_{0, m-1}\left(\nu_{m}\right)+e_{A}\left(d\left(\nu_{m}\right)\right)$, where $e \in$ $E(2, n), e(1)=m-1, e(2)=m$, and $e_{A}\left(d\left(\nu_{m}\right)\right)=\Sigma \delta_{0, m-1}\left(\nu_{i}^{\prime}\right) \delta_{m-1, m}\left(\nu_{i}^{\prime \prime}\right)$, we see that $\delta_{0, m-1}\left(\nu_{m-1}\right) \delta_{m-1, m}\left(\nu_{m}\right)=\delta_{0, m-1}\left(\nu_{m-1}\right) \delta_{0, m}\left(\nu_{m}\right)$, hence the assertion.

${ }^{24}$ I.e., dg algebras which are commutative up to a coherent system of higher homotopies. See [HS] for a nice, if old-fashioned, initial exposition (which explains, in particular, the $\mathrm{E}_{\infty}$ algebra structure on the log crystalline complexes defined explicitly using the Godement resolution).
} 
(ii) For any pd-S $S^{\sharp}$-smooth $(Z, P) \in(Z / S)_{\text {crys }}^{\log }$ the natural map $R u_{Z / S *}^{\log }\left(\Omega_{Z / S} \mathcal{F}\right) \rightarrow\left(\Omega_{Z / S} \mathcal{F}\right)_{(Z, P)}$ is a quasi-isomorphism. Thus (cf. [K1] 6.4 )

$$
R u_{Z / S *}^{\log }(\mathcal{F}) \stackrel{\sim}{\rightarrow}\left(\Omega_{Z / S} \mathcal{F}\right)_{(Z, P)}
$$

Proof. The assertions are local, so we can assume that we have $(Z, P)$ as in (ii) and $(Z, \mathcal{M})$ is $\log$ affine (see 1.1$)$. Pick a pd- $S^{\sharp}$-smooth $(Z, T)$ (say, a copy of $(Z, P))$. Let $\left(Z, Q_{*}\right)$ be the product of $\left(Z, T_{*}\right)$ and $(Z, P)$; this is a simplicial object of $(Z / S)_{\text {crys }}^{\log }$ augmented over $(Z, P)$. Consider the total complex of the cosimplicial complex $\left(\Omega_{Z / T_{*}} \mathcal{F}\right)_{\left(Z, Q_{*}\right)}$. Let us show that the pullback maps are quasi-isomorphisms:

$$
(\mathcal{C} \cdot \mathcal{F})_{(Z, T)}=\mathcal{F}_{\left(Z, T_{*}\right)} \stackrel{\sim}{\rightarrow}\left(\Omega_{Z / T_{*}} \mathcal{F}\right)_{\left(Z, Q_{*}\right)} \stackrel{\sim}{\leftarrow}\left(\Omega_{Z / S} \mathcal{F}\right)_{(Z, P)} .
$$

First arrow: Let us check that the maps $\mathcal{F}_{\left(Z, T_{i}\right)} \rightarrow\left(\Omega_{Z / T_{i}} \mathcal{F}\right)_{\left(Z, Q_{i}\right)}$ are quasi-isomorphisms. By Remark (i) in 1.4, $(Z, P)$ is a retract of a coordinate pd- $S^{\sharp}$-thickening $\left(Z, P^{\prime}\right)$. Let $\left(Z, Q_{i}^{\prime}\right)$ be the product of $\left(Z, T_{i}\right)$ and $\left(Z, P^{\prime}\right)$. Then $\Omega_{\left(Z, Q_{i}\right) / T_{i}}$ is a retract of $\Omega_{\left(Z, Q_{i}^{\prime}\right) / T_{i}}$, so the assertion for $\Omega_{\left(Z, Q_{i}^{\prime}\right) / T_{i}}$ implies that for $\Omega_{\left(Z, Q_{i}\right) / T_{i}}$ (since the map $\mathcal{F}_{\left(Z, T_{i}\right)} \rightarrow H^{0}\left(\Omega_{Z / T_{i}} \mathcal{F}\right)_{\left(Z, Q_{i}\right)}$ is injective). By Remark (i) in 1.6, $\left(Z, Q_{i}^{\prime}\right)$ is a $\mathbb{G}_{m}^{\sharp I} \times \mathbb{G}_{a}^{\sharp J}$-torsor over $\left(Z, T_{i}\right)$, and we are done by the evident computation of the de Rham pd-complexes of $\mathbb{G}_{m}^{\sharp}$ and $\mathbb{G}_{a}^{\sharp}$.

Second arrow: Let us check that the maps $\left(\Omega_{Z / S}^{a} \mathcal{F}\right)_{(Z, P)} \rightarrow\left(\Omega_{Z / T_{*}}^{a} \mathcal{F}\right)_{\left(Z, Q_{*}\right)}$ are quasi-isomorphisms. For case $a=0$, see the proof of the proposition in 1.6; the general case follows by base change since $\Omega_{(Z, P) / S}^{a}$ is $\mathcal{O}_{P}$-flat by Exercise (ii) in 1.7.

Since diagrams (1.8.2) are compatible with maps between $P$ 's, we see that the simplicial structure maps $\left(\Omega_{Z / S} \mathcal{F}\right)_{\left(Z, P_{i}\right)} \rightarrow\left(\Omega_{Z / S} \mathcal{F}\right)_{\left(Z, P_{j}\right)}$ are quasiisomorphisms. Now both (ii) and (i) of the theorem follow from the proposition in 1.6 , q.e.d.

Remarks. (i) If no global $(Z, P)$ as in above is available, then one can compute $R u_{Z / S *}^{\log }(\mathcal{F})$ using $(1.8 .1)$ and Remark (iii) in 1.6.

(ii) If $(S, \mathcal{L})$ is fine and $(Z, \mathcal{M})$ is $\log$ smooth over $(S, \mathcal{L})$, then $(1.8 .1)$ implies

$$
R u_{Z / S *}^{\log }(\mathcal{F}) \stackrel{\sim}{\rightarrow}\left(\Omega_{Z / S} \mathcal{F}\right)_{(Z, \mathcal{M})}
$$


(iii) The map $\Omega_{Z / S} \rightarrow \mathcal{O}_{Z / S}$ sends $F^{m} \Omega_{Z / S} \mathcal{F}$ to $\mathcal{J}_{Z / S}^{[m]} \mathcal{F}$ (see 1.7 for the notation). The quasi-isomorphisms in the statement of the proposition are, in fact, filtered quasi-isomorphisms for these filtrations. The proof is the same just replace "quasi-isomorphism" in it by "filtered quasi-isomorphism".

(iv) Quasi-isomorphism (1.8.1) coincides with the composition of maps in (1.8.2) and, via (1.6.1) and 1.7, with (the normalization of) the restriction map of cosimplicial sheaves $\mathcal{F}_{P_{*}} \rightarrow \mathcal{F}_{P_{*}^{b}}$.

1.9. Comparison with derived de Rham cohomology. We discuss log version of Illusie's comparison theorem [Ill2] Ch. VIII, 2.2.8 due to Olsson [Ol1] 6.10.

Let $L \Omega_{(Z, \mathcal{M}) /(S, \mathcal{L})}$ be the derived log de Rham complex (we use Gabber's construction, [Ol1] $\S 8$ or [Bh2] $\S 6$, to be recalled in a moment; see [B] 3.1 for a short review and the notation used below). This is a commutative dg $\mathcal{O}_{S}$-algebra on $Z_{\text {ét }}$ equipped with the Hodge filtration $F^{m}$. Let us define a natural morphism of filtered commutative $\operatorname{dg} \mathcal{O}_{S}$-algebras

$$
L \Omega_{(Z, \mathcal{M}) /(S, \mathcal{L})} \rightarrow R u_{Z / S *}^{\log }\left(\mathcal{O}_{Z / S}\right)
$$

Assume for simplicity that $S=\operatorname{Spec} A, \mathcal{L}$ comes from a prelog structure $L \rightarrow A \cdot{ }^{25}$ For a $\log$ affine $U \in Z_{\text {ét }}$ set $B:=\Gamma\left(U, \mathcal{O}_{U}\right), M:=\Gamma\left(U, \mathcal{M}_{U}\right)$, so we have $(B, M) /(A, L) \in \mathcal{C}_{(A, L)}$. Let $P(U)$. $=P_{(A, L)}(B, M)$. be its canonical simplicial resolution (see [Ol1] 8.3), and $\Omega_{P(U) . /(A, L)}$ be the relative log de Rham complex, which is a simplicial dg algebra. Let $L \Omega_{(B, M) /(A, L)}$ be the total complex, $L \Omega_{(B, M) /(A, L)}^{n}=\oplus_{i} \Omega_{P(U)_{i-n} /(A, L)}^{i}$, filtered by the Hodge filtration $F^{m}:=\oplus_{i \geq m} \Omega_{P(U)_{i-n} /(A, L)}^{i}$. We have the filtered complex of presheaves $U \mapsto L \Omega_{(B, M) /(A, L)}$ on $Z_{\mathrm{é}^{\prime}}$; the associated filtered complex of sheaves is quasi-isomorphic to $L \Omega_{(Z, \mathcal{M}) /(S, \mathcal{L})}$.

Let $\left(U, T^{a}\right)$ be the $\operatorname{pd}-S^{\sharp}$-completion of the embedding $\left(U, \mathcal{M}_{U}\right) \hookrightarrow$ Spec $P(U)_{a} ;{ }^{26}$ this is a cosimplicial object in $(Z / S)_{\text {crys. }}^{\log }$ Each $\left(U, T^{a}\right)$ is pd$S^{\sharp}$-smooth (see Remark (i) in 1.4), so one has the filtered quasi-isomorphism $\left.R u_{Z / S *}^{\log }\left(\mathcal{O}_{Z / S}\right)\right|_{U_{\text {ét }}} \stackrel{\sim}{\rightarrow} \Omega_{\left(U, T^{a}\right) / S}$ (see 1.8). Let $\Omega_{Z / S}^{\natural}$. $(U)$ be the total complex of the simplicial dg algebra $\Omega_{Z / S}\left(U, T^{\cdot}\right), \Omega_{Z / S}^{\sharp n}(U)=\oplus_{i} \Omega_{Z / S}^{i}\left(U, T^{i-n}\right)$, filtered by the Hodge-pd filtration. We see that $\Omega_{Z / S}\left(U, T^{0}\right) \hookrightarrow \Omega_{Z / S}^{\natural \cdot}(U)$ is a filtered quasi-isomorphism, so $R u_{Z / S *}^{\log }\left(\mathcal{O}_{Z / S}\right)$ is represented by the filtered complex of presheaves $U \mapsto \Omega_{Z / S}^{\natural}(U)$.

\footnotetext{
${ }^{25}$ Otherwise do the construction étale locally on $S$.

${ }^{26}$ Notice that $\left(U, T^{0}\right)$ is $\left(U, P^{\text {univ }}\right)$ from Remark (iii) in 1.4 .
} 
Now the de Rham pullback $r^{*}: \Omega_{P(U) . /(A, L)} \rightarrow \Omega_{Z / S}\left(U, T^{*}\right)$ for the map of $\log$ schemes $r: T^{*} \rightarrow \operatorname{Spec} P(U)$. (see (1.3.1)) sends the Hodge filtration to the Hodge-pd one, and (1.9.1) is the map between the total complexes.

Theorem. Suppose $(Z, \mathcal{M}),(S, \mathcal{L})$ are fine and $f:(Z, \mathcal{M}) \rightarrow(S, \mathcal{L})$ is an integral locally log complete intersection map. Then (1.9.1) yields quasiisomorphisms

$$
L \Omega_{(Z, \mathcal{M}) /(S, \mathcal{L})} / F^{m} \stackrel{\sim}{\rightarrow} R u_{Z / S *}^{\log }\left(\mathcal{O}_{Z / S} / \mathcal{J}_{Z / S}^{[m]}\right) .
$$

Proof. (a) Let $f:(Z, \mathcal{M}) \rightarrow(S, \mathcal{L})$ be a map of fine log schemes. Recall (see [KS] 4.4.2, 4.4.4 or [Ol1] 6.8) that $f$ is locally log complete intersection map if étale locally it can be factored as $(Z, \mathcal{M}) \stackrel{i}{\rightarrow}\left(Y, M_{Y}\right) \stackrel{g}{\rightarrow}(S, \mathcal{L})$ with $g$ $\log$ smooth, $i$ an exact closed embedding, and $Z \hookrightarrow Y$ a regular immersion. Then, by loc. cit., for any other factorization $f=g^{\prime} i^{\prime}$ with the first two properties, the third one holds automatically. We say (see [K1] 4.1, 4.3) that $f$ is integral at a closed point $z \in Z$ if the map $\mathbb{Z}\left[\left(\mathcal{L} / \mathcal{O}_{S}^{\times}\right)_{f(z)}\right] \rightarrow \mathbb{Z}\left[\left(\mathcal{M} / \mathcal{O}_{Z}^{\times}\right)_{z}\right]$ induced by $f$ is flat, and $f$ is integral if it is integral at every $z \in Z$. If $f$ is integral at $z$, then it is integral on an étale neighborhood of $z \cdot{ }^{27}$ So for $f$ as in Theorem the local factorization $f=g i$ can be chosen with $g$ integral (so $Y$ is flat over $S$ by [K1] 4.5).

(b) We return to the theorem. Its assertion is local, so, combining [K1] $3.5,4.1$, we can assume that there is a fine chart $L \rightarrow M$ for $f$ (see 1.1) such that $\mathbb{Z}[M]$ is $\mathbb{Z}[L]$-flat, the map $L^{\mathrm{gr}} \rightarrow M^{\mathrm{gr}}$ is injective with the cokernel having prime to $p$ torison, and the map $i: Z \rightarrow Y:=\operatorname{Spec}\left(\mathbb{Z}[M] / p^{n}\right) \times_{\operatorname{Spec}\left(\mathbb{Z}[L] / p^{n}\right)}$ $S$ is a regular immersion with parameters $t_{1}, \ldots, t_{m} \in \mathcal{O}_{Y}$. Let $\mathcal{M}_{Y}$ be the $\log$ structure on $Y$ defined by the chart $M$, and $\mathcal{M}^{\prime}, \mathcal{L}^{\prime}$ be the log structures on $\operatorname{Spec}\left(\mathbb{Z}[M] / p^{n}\right)$, Spec $\left(\mathbb{Z}[L] / p^{n}\right)$ defined by $M, L$. The log cotangent $\operatorname{complex~}_{\left(\operatorname{Spec}\left(\mathbb{Z}[M] / p^{n}\right), \mathcal{M}^{\prime}\right) /\left(\operatorname{Spec}\left(\mathbb{Z}[L] / p^{n}\right), \mathcal{L}^{\prime}\right)}$ equals $\mathrm{L}_{\left(\mathbb{Z}[M] / p^{n}, M\right) /\left(\mathbb{Z}[L] / p^{n}, L\right)} \stackrel{\sim}{\rightarrow}$ $\left(\mathbb{Z}[M] / p^{n}\right) \otimes\left(M^{\mathrm{gr}} / L^{\mathrm{gr}}\right)$. By base change (we use the flatness), $\mathrm{L}_{\left(Y, \mathcal{M}_{Y}\right) /(S, \mathcal{L})} \stackrel{\sim}{\rightarrow}$ $\Omega_{\left(Y, \mathcal{M}_{Y}\right) /(S, \mathcal{L})}^{1} \stackrel{\sim}{\rightarrow} \mathcal{O}_{Y} \otimes\left(M^{\mathrm{gr}} / L^{\mathrm{gr}}\right)$, where $d \log \left(c_{M}(m)\right)$ is identified with $\alpha c_{M}(m) \otimes m$ (see 1.1 for the notation). One has (see [Ol1] 8.22) $\mathrm{L}_{(Z, \mathcal{M}) /\left(Y, \mathcal{M}_{Y}\right)}=\mathrm{L}_{Z / Y} \stackrel{\sim}{\rightarrow} \mathcal{O}_{Z}^{m}[1]$, the generators $e_{1}, \ldots, e_{m}$ of $\mathcal{O}_{Z}^{m}$ correspond to regular parameters $t_{1}, \ldots, t_{m} \in \mathcal{O}_{Y}$. By the transitivity, one

\footnotetext{
${ }^{27}$ Proof: We can assume that $f$ admits a fine chart $c_{M}: M \rightarrow \Gamma(Z, \mathcal{M})$, etc., as in 1.1, and for any $m \in M$ the function $\alpha c_{M}(m) \in \Gamma\left(Z, \mathcal{O}_{Z}\right)$ is invertible if it is invertible at $z$. Then $f$ is integral. This follows from [K1] 4.1 and the next observation: Let $h: Q \rightarrow P$ be a map of integral monoids that satisfies condition (iv) of [K1] 4.1(i). Then for any submonoid $P^{\prime} \subset P$ the map of the integral monoid quotients $Q / h^{-1}\left(P^{\prime}\right) \rightarrow P / P^{\prime}$ satisfies the same condition (iv).
} 
has $\mathrm{L}_{(Z, \mathcal{M}) /(S / \mathcal{L})}=\operatorname{Cone}\left(\delta: \mathcal{O}_{Z}^{m} \rightarrow \Omega_{(Y, \mathcal{M}) /(S, \mathcal{L})}^{1} \mid Z\right), \delta\left(e_{i}\right)=\left.d t_{i}\right|_{Z}$, so $\operatorname{gr}_{F} L \Omega_{(Z, \mathcal{M}) /(S, \mathcal{L})}=L \Lambda \cdot \mathcal{C}$ one $(\delta)$. By [Ill1] Ch. I, 4.3.2.1(ii), this is equal to $\mathcal{O}_{Z}\left\langle e_{1}, \ldots, e_{m}\right\rangle \otimes_{\mathcal{O}_{Y}} \Omega_{\left(Y, \mathcal{M}_{Y}\right) /(S, \mathcal{L})}$, where the first factor is the divided powers polynomial algebra.

We compute $R u_{Z / S *}^{\log }\left(\mathcal{O}_{Z / S}\right)$ using (1.8.1) with $(Z, P)$ equal to the pdenvelope of $i:(Z, \mathcal{M}) \hookrightarrow(Y, \mathcal{M})$. Since $i$ is exact, $P$ is the pd-envelope of $Z \hookrightarrow Y$, so $\oplus \mathcal{J}_{P}^{[m]} / \mathcal{J}_{P}^{[m+1]} \stackrel{\sim}{\rightarrow} \mathcal{O}_{Z}\left\langle e_{1}, \ldots, e_{m}\right\rangle$. So, by Remark (iii) in $1.8, \operatorname{gr}_{F}^{*} R u_{Z / S *}^{\log }\left(\mathcal{O}_{Z / S}\right) \stackrel{\sim}{\rightarrow} \mathcal{O}_{Z}\left\langle e_{1}, \ldots, e_{m}\right\rangle \otimes_{\mathcal{O}_{Y}} \Omega_{(Y, \mathcal{M}) /(S, \mathcal{L})}$. The above two identifications provide an isomorphism of dg algebras $\operatorname{gr}_{F} L \Omega_{(Z, \mathcal{M}) /(S, \mathcal{L})} \stackrel{\sim}{\rightarrow}$ $\operatorname{gr}_{F}^{\circ} R u_{Z / S *}^{\log }\left(\mathcal{O}_{Z / S}\right)$, which clearly coincides with the associated graded isomorphism to (1.9.1), q.e.d.

Remarks. (i) The assertion remains true, by the direct limit argument, if $(Z, \mathcal{M})$ is projective limit of $\log (S, \mathcal{L})$-schemes $\left(Z_{\alpha}, \mathcal{M}_{\alpha}\right)$ as in the theorem with respect to a directed family of affine transition maps.

(ii) In the original theorem [Ol1] 6.10, Olsson uses his version of log cotangent complex; there $f$ need not be integral. For integral $f$ Olsson's version of log cotangent complex coincides with Gabber's one (that we use) by [Ol1] 8.34. (iii) By Bhatt [Bh2] 7.22, (1.9.1) is itself a quasi-isomorphism if, in addition, $f \otimes \mathbb{Z} / p$ is of Cartier type and $Z, S$ are $\mathbb{Z} / p^{n}$-flat. We will not use this result.

1.10. The Cartier isomorphism. Suppose our $S$ is an $\mathbb{F}_{p}$-scheme. Let $(Y, \mathcal{N})$ be any $\log S^{\sharp}$-scheme. Then for any $(V, T) \in(Y / S)_{\text {crys }}^{\log }$ the Frobenius map $F r_{T}$ kills $\mathcal{J}_{T}$ since it is a pd-ideal, i.e., $F r_{T}$ factors as $T \rightarrow V \hookrightarrow T$; denote the first arrow by $F r_{T}^{\prime}$. The datum of all maps $F r_{T}^{\prime}$ forms an extension of the canonical morphism of topoi $u_{Y / S}^{\log }:(Y / S)_{\text {crys }}^{\log \sim} \rightarrow Y_{\text {ét }}$ (see 1.5) to a morphism $F r_{Y}^{\prime}$ of the ringed topoi.

Let $f:(Z, \mathcal{M}) \rightarrow(Y, \mathcal{N})$ be a log smooth map of Cartier type between fine $\log S^{\sharp}$-schemes; assume that $Z, Y$ are quasi-compact and quasiseparated.

Theorem. The $\mathcal{O}_{Y / S}$-complex $R f_{\text {crys* }} \mathcal{O}_{Z / S}$ carries a natural finite filtration con., called the conjugate filtration, together with canonical Cartier quasiisomorphisms

$$
C=C_{f}^{q}: g r_{q}^{\text {con }} R f_{\text {crys* }} \mathcal{O}_{Z / S} \stackrel{\sim}{\rightarrow} F r_{Y}^{* *} R f_{*} \Omega_{(Z, \mathcal{M}) /(Y, \mathcal{N})}^{q}[-q] .
$$

Remark. The conditions imply that $\Omega_{(Z, \mathcal{M}) /(Y, \mathcal{N})}^{q}$ are locally free $\mathcal{O}_{Z}$-modules of finite rank, $f$ is flat, and $R f_{*} \Omega_{(Z, \mathcal{M}) /(Y, \mathcal{N})}^{q}$ are quasi-coherent of finite Tordimension. 
Proof. For $(V, T)$ as above, one has $\left(R f_{\text {crys* }} \mathcal{O}_{Z / S}\right)(V, T)=R \Gamma\left(\left(Z_{V} / T\right)_{\text {crys }}^{\log \text {, }}\right.$ $\left.\mathcal{O}_{Z_{V} / T}\right)=R \Gamma\left(Z_{V}, R u_{Z_{V} / T}^{\log } \mathcal{O}_{Z_{V} / T}\right)$. The canonical filtration on $R u_{Z_{V} / T}^{\log } \mathcal{O}_{Z_{V} / T}$ yields thus a filtration $\operatorname{con}_{(V, T)}$. on $\left(R f_{\text {crys* }} \mathcal{O}_{Z / S}\right)(V, T)$. The filtrations $\operatorname{con}_{(V, T)}$. are compatible with morphisms of $(V, T)$. They form the promised conjugate filtration con..

Identifications (1.10.1) have local nature: they come from isomorphisms

$$
C: H^{q} R u_{Z_{V} / T *}^{\log }\left(\mathcal{O}_{Z_{V} / T}\right) \stackrel{\sim}{\rightarrow} \Omega_{\left(Z_{V}, \mathcal{M}\right) /(V, \mathcal{N})}^{q} \otimes_{f_{V}^{-1} \mathcal{O}_{V}} f_{V}^{-1} \mathcal{O}_{T}
$$

where $\otimes$ in the r.h.s. is taken for the map $F r_{T}^{* *}: \mathcal{O}_{V} \rightarrow \mathcal{O}_{T}$, by applying $R \Gamma\left(Z_{V}, \cdot\right)$.

To define (1.10.2), let us find a map $C^{-1}: \Omega_{\left(Z_{V}, \mathcal{M}\right) /(V, \mathcal{N})}^{q} \otimes_{f_{V}^{-1} \mathcal{O}_{V}} f_{V}^{-1} \mathcal{O}_{T} \rightarrow$

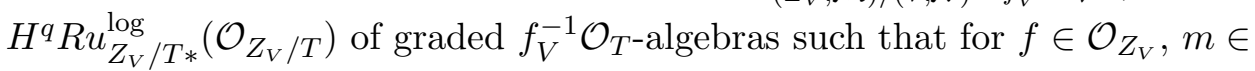
$\mathcal{M}$ one has $C^{-1}(f)=f^{*}\left(t^{p}\right), C^{-1}(d \log m)=m^{*}(d \log t)$. Here $f, m$ at the r.h.s. are viewed as the $\mathbb{A}_{\left(T, \mathcal{N}_{T}\right)}^{1}$ and $\mathbb{A}_{\left(T, \mathcal{N}_{T}\right)}^{(1)}$-valued maps, and $t^{p}, d \log t$ are the usual cohomology classes in $H_{\mathrm{dR}}^{0}\left(\mathbb{A}_{\left(T, \mathcal{N}_{T}\right)}^{1} /\left(T, \mathcal{N}_{T}\right)\right)=H^{0} R \Gamma\left(\left(\mathbb{A}_{\left(T, \mathcal{N}_{T}\right)}^{1} /\right.\right.$ $\left.\left.\left(T, \mathcal{N}_{T}\right)\right)_{\text {crys }}, \mathcal{O}_{\mathbb{A}_{\left(T, \mathcal{N}_{T}\right)}^{1} / T}\right)$ and $H_{\mathrm{dR}}^{1}\left(\mathbb{A}_{\left(T, \mathcal{N}_{T}\right)}^{(1)} /\left(T, \mathcal{N}_{T}\right)\right)=H^{1} R \Gamma\left(\left(\mathbb{A}_{\left(T, \mathcal{N}_{T}\right)}^{(1)} /\right.\right.$ $\left.\left(T, \mathcal{N}_{T}\right)\right)_{\text {crys }}, \mathcal{O}_{\mathbb{A}_{\left(T, \mathcal{N}_{T}\right)}^{1} / T}$ ) (see 1.1, (1.8.3)). The above properties determine $C^{-1}$ uniquely, so it is enough to find one such $C^{-1}$ locally. By Remark (ii) in 1.1 , we can assume that $\left(Z_{V}, \mathcal{M}\right)$ extends in a $\log$ smooth way over $\left(T, \mathcal{N}_{T}\right)$. Now we are in the setting of [K1] 4.12(1), which provides (via (1.8.3)) $C^{-1}$. By loc. cit., $C^{-1}$ is an isomorphism, and $C$ of (1.10.2) is its inverse.

1.11. Perfect crystals and base change. We are in general situation of 1.5 , so $S^{\sharp}$ is as in 1.3. Let $(Y, \mathcal{N})$ be any integral quasi-coherent $\log S^{\sharp}$-scheme.

A bounded complex $\mathcal{F}$ of $\mathcal{O}_{Y / S^{-m o d u l e s ~ o n ~}}(Y / S)_{\text {crys }}^{\log }$ is an $\mathcal{O}_{Y / S}$-crystal (in derived sense) if for every $\phi:\left(V^{\prime}, T^{\prime}\right) \rightarrow(V, T)$ in $(Y / S)_{\text {crys }}^{\log }$ the pullback map $\phi^{-1} \mathcal{F}_{(V, T)} \rightarrow \mathcal{F}_{\left(V^{\prime}, T^{\prime}\right)}$ yields a quasi-isomorphism of $\mathcal{O}_{T^{\prime}}$-complexes $L \phi^{*} \mathcal{F}_{(V, T)} \stackrel{\sim}{\rightarrow} \mathcal{F}_{\left(V^{\prime}, T^{\prime}\right)}$. We say that $\mathcal{F}^{*}$ is perfect if for every $(V, T) \in(Y / S)_{\text {crys }} \log _{\text {of }}$ the complex $\mathcal{F}_{(V, T)}$ is $\mathcal{O}_{T}$-perfect. Perfect crystals form a full triangulated subcategory $D^{\mathrm{pcr}}(Y / S)$ of the derived category $D^{b}\left((Y / S)_{\mathrm{crys}}^{\log }, \mathcal{O}_{Y / S}\right)$ of $\mathcal{O}_{Y / S^{-}}$ modules.

Let now $\theta:\left(Y^{\nu}, \mathcal{N}^{\nu}\right) / S^{\nu \sharp} \rightarrow(Y, \mathcal{N}) / S^{\sharp}$ be a map of data as above. Exercise. If $\mathcal{F}$ is a (perfect) $\mathcal{O}_{Y / S^{-c r y s t a l}}$ then $L \theta_{\text {crys }}^{*}\left(\mathcal{F}^{\cdot}\right)$ is a (perfect) $\mathcal{O}_{Y^{\nu} / S^{\nu}}$-crystal. For any $(V, T) \in(Y / S)_{\text {crys }}^{\log },\left(V^{\nu}, T^{\nu}\right) \in\left(Y^{\nu} / S^{\nu}\right)_{\text {crys }}^{\log }$, and a map of log pd-thickenings $\theta_{T^{\nu} / T}:\left(V^{\nu}, T^{\nu}\right) \rightarrow(V, T)$ compatible with $\theta$, one has a canonical identification $L \theta_{\text {crys }}^{*}\left(\mathcal{F}^{\cdot}\right)_{\left(V^{\nu}, T^{\nu}\right)} \stackrel{\sim}{\rightarrow} L \theta_{T^{\nu} / T}^{*}\left(\mathcal{F}_{(V, T)}^{\cdot}\right)$. 
The next version of base change theorem [K1] 6.10 is sufficient for our purposes. Let $f:(Z, \mathcal{M}) \rightarrow(Y, \mathcal{N})$ be a $\log$ smooth integral map of fine $\log$ schemes over $S_{1}^{\sharp}:=S^{\sharp} \otimes \mathbb{F}_{p}$; assume that $Z / Y$ is quasi-compact and separated. For $\theta$ as above, let $f^{\nu}:\left(Z^{\nu}, \mathcal{M}^{\nu}\right) \rightarrow\left(Y^{\nu}, \mathcal{N}^{\nu}\right)$ be the $\theta$-pullback of $f$. Then $Z$ is flat over $Y$ (by [K1] 4.5), $Z^{\nu}$ is flat over $Y^{\nu}$, and $\left(Z^{\nu}, \mathcal{M}^{\nu}\right)$ is integral quasi-coherent (by [K1] 4.3.1 and Exercise (ii) in 1.1).

Theorem. (i) The complex $R f_{\text {crys* }}\left(\mathcal{O}_{Z / S}\right)$ is an $\mathcal{O}_{Y / S^{-}}$crystal, and the pullback map yields a canonical identification

$$
L \theta_{\text {crys }}^{*} R f_{\text {crys* }}\left(\mathcal{O}_{Y / S}\right) \stackrel{\sim}{\rightarrow} R f_{\text {crys* }}^{\nu}\left(\mathcal{O}_{Z^{\nu} / S^{\nu}}\right) .
$$

(ii) If $Z$ is proper over $Y$ and $f$ is of Cartier type, then $R f_{\text {crys* }}\left(\mathcal{O}_{Z / S}\right)$ is perfect.

Proof. (i) We can assume that $Y$ is affine. Computing $R f_{\text {crys* }}\left(\mathcal{O}_{Z / S}\right)$ using a finite covering of $Z$ by affine opens, we get a finite filtration on it with gr. $R f_{\text {crys* }}\left(\mathcal{O}_{Z / S}\right)$ equal to a finite direct sum of complexes of type $R\left(\left.f\right|_{U}\right)_{\text {crys* }}\left(\mathcal{O}_{U / S}\right), U \subset Z$ is an affine open. Thus we can assume that $Z$ is affine. Using Remark (ii) in 1.1 and (1.8.3), we see that each $R f_{\text {crys* }}\left(\mathcal{O}_{Z / S}\right)_{(V, T)}$ can be realized as a finite complex of flat $\mathcal{O}_{T}$-modules, for every $\phi:\left(V^{\prime}, T^{\prime}\right) \rightarrow(V, T)$ in $(Y / S)_{\text {crys }}^{\log }$ the pullback map yields a quasiisomorphism $L \phi^{*} R f_{\text {crys* }}\left(\mathcal{O}_{Z / S}\right)_{(V, T)} \stackrel{\sim}{\rightarrow} R f_{\text {crys* }}\left(\mathcal{O}_{Z / S}\right)_{\left(V^{\prime}, T^{\prime}\right)}$, and the pullback map for $\theta$ yields quasi-isomorphism (1.11.1).

(ii) Let us check that the complexes $R f_{\text {crys* }}\left(\mathcal{O}_{Z / S}\right)_{(V, T)}$ are $\mathcal{O}_{T}$-perfect. By (i), $R f_{\text {crys* }}\left(\mathcal{O}_{Z / S}\right)_{(V, T)} \otimes_{\mathcal{O}_{T}}^{L} \mathcal{O}_{T_{1}}=R f_{\text {crys* }}\left(\mathcal{O}_{Z / S_{1}}\right)_{\left(V, T_{1}\right)}$, where $T_{1}:=T \otimes$ $\mathbb{F}_{p}$. Since $p$ is nilpotent in $\mathcal{O}_{T}$, it suffices to check that $R f_{\text {crys* }}\left(\mathcal{O}_{Z / S_{1}}\right)_{\left(V, T_{1}\right)}$ is $\mathcal{O}_{T_{1}}$-perfect, which follows from (1.10.1).

Remarks. (a) If $(V, T) \in(Y / S)_{\text {crys }}^{\log }$ is such that $\mathcal{J}_{T}^{[n]}=0$, then $R f_{\text {crys* }}\left(\mathcal{O}_{Z / S}\right)_{(V, T)} \stackrel{\sim}{\rightarrow} R f_{\text {crys* }}\left(\mathcal{O}_{Z / S} / \mathcal{J}_{Z / S}^{[m]}\right)_{(V, T)}$ for $m \geq n+\operatorname{dim} Z / Y{ }^{28}$

(b) If we drop the Cartier type assumption in (ii), then $R f_{\text {crys* }}\left(\mathcal{O}_{Z / S}\right)_{(V, T)}$ is still $\mathcal{O}_{T}$-perfect for those $(V, T)$ that the ideal $\mathcal{J}_{T}$ is nilpotent. ${ }^{29}$ In particular, $R f_{\text {crys* }}\left(\mathcal{O}_{Z / S}\right)_{(Y, Y)}$ is $\mathcal{O}_{Y}$-perfect. So $R f_{\text {crys* }}\left(\mathcal{O}_{Z / S}\right)$ is perfect if $Y=S$ (use (i)).

\footnotetext{
${ }^{28}$ Proof: As in the proof of (i), we can assume that $Z, Y, V$ are affine. Then $(Z, \mathcal{M})_{V}$ can be extended to a log smooth scheme over $\left(T, \mathcal{N}_{T}\right)$ by [K1] 3.14. Now use Remark (iii) in 1.8.

${ }^{29}$ Proof: It suffices, by the nilpotency, to check that $R f_{\text {crys* }}\left(\mathcal{O}_{Z / S}\right)_{(V, T)} \otimes{ }^{L} \mathcal{O}_{V}$ is $\mathcal{O}_{V}$-perfect; this complex equals $R f_{\text {crys* }}\left(\mathcal{O}_{Z / S}\right)_{(V, V)}=\left(R f_{*} \Omega_{(Z, \mathcal{M}) /(Y, \mathcal{N})}\right)_{V}$ by (i) and 1.8 , which is perfect.
} 
1.12. The p-adic setting; absolute crystalline cohomology. One can generalize slightly the setting for log crystalline cohomology taking for $S^{\sharp}=$ $(S, \mathcal{L}, \mathcal{I})$ a formal $p$-adic log pd-scheme, which is the same as sequence of exact closed embeddings of $\log$ pd-schemes $S_{1}^{\sharp} \hookrightarrow S_{2}^{\sharp} \hookrightarrow \ldots$ such that $\mathcal{O}_{S_{n-1}}=\mathcal{O}_{S_{n}} \otimes \mathbb{Z} / p^{n-1}, \mathcal{I}_{n-1}=\mathcal{I}_{n} \mathcal{O}_{S_{n-1}}$. Assume that $(S, \mathcal{L})$ is quasicoherent, i.e., all $\left(S_{n}, \mathcal{L}_{n}\right)$ are quasi-coherent. For an integral quasi-coherent $\log S^{\sharp}$-scheme $(Z, \mathcal{M})$, which is a $\log S_{n}^{\sharp}$-scheme for $n$ sufficiently large, one defines its $\log$ crystalline site $(Z / S)_{\text {crys }}^{\log }$ as in 1.5 . One has fully faithful embeddings $\left(Z / S_{n}\right)_{\text {crys }}^{\log } \hookrightarrow\left(Z / S_{n+1}\right)_{\text {crys }}^{\log } \hookrightarrow \ldots$, and $(Z / S)_{\text {crys }}^{\log }=\cup\left(Z / S_{n}\right)_{\text {crys }}^{\log }$. The constructions and results of 1.5 remain true in the present setting.

For a sheaf $\mathcal{F}$ on $(Z / S)_{\text {crys }}^{\log }$ we denote by $\mathcal{F}_{(n)}$ its restriction to $\left(Z / S_{n}\right)_{\text {crys }}^{\log }$. Then $\mathcal{F}_{(n)}=i_{n \text { crys }}^{*}(\mathcal{F})$, where $i_{n}:(Z, \mathcal{M}) / S_{n}^{\sharp} \rightarrow(Z, \mathcal{M}) / S^{\sharp}$ is the evident map. The functor $i_{n \text { crys }}^{*}$ is exact, and it admits an evident exact left adjoint $i_{n \text { crys! }}$, so $i_{n \text { crys }}^{*}$ sends injective sheaves to injective ones. Therefore the functors $R f_{\text {crys* }}, L f_{\text {crys }}^{*}$ commute with the passage $\mathcal{F} \mapsto$ $\mathcal{F}_{(n)}$, and one has $R u_{Z / S *}^{\log }(\mathcal{F})=\operatorname{holim}_{n} R u_{Z / S_{n} *}^{\log }\left(\mathcal{F}_{(n)}\right), R \Gamma\left((Z / S)_{\text {crys }}^{\log }, \mathcal{F}\right)=$ $\operatorname{holim}_{n} R \Gamma\left(\left(Z / S_{n}\right)_{\text {crys }}^{\log }, \mathcal{F}_{(n)}\right)$.

Example. For a complex $\mathcal{F}$ of sheaves on $\left.\left(S_{1}, S\right)\right)_{\text {crys }}^{\log }$, one has $R \Gamma\left(\left(S_{1}, S_{n}\right)_{\text {crys }}^{\log \text {, }}\right.$ $\left.\mathcal{F}_{(n)}\right)=\mathcal{F} \cdot\left(S_{1}, S_{n}\right), R \Gamma\left(\left(S_{1}, S\right)_{\text {crys }}^{\log }, \mathcal{F}\right)=\operatorname{holim}_{n} \mathcal{F} \cdot\left(S_{1}, S_{n}\right)$. Unless $\mathcal{F}$ is a crystal, the base change map $R \Gamma\left(\left(S_{1}, S\right)_{\text {crys }}^{\log }, \mathcal{F}^{\cdot}\right) \otimes{ }^{L} \mathbb{Z} / p^{n} \rightarrow R \Gamma\left(\left(S_{1}, S_{n}\right)_{\text {crys }}^{\log }\right.$, $\left.\mathcal{F}_{(n)}\right)$ need not be a quasi-isomorphism.

Consider now $S_{n}^{\sharp}=\operatorname{Spec}\left(\mathbb{Z} / p^{n}\right)$ equipped with the trivial log structure and $\mathcal{I}_{n}=p\left(\mathbb{Z} / p^{n}\right)$ with the standard pd structure. Any integral quasicoherent $\log \mathbb{F}_{p^{-} \text {-scheme }}(Z, \mathcal{M})$ is automatically a $\log S^{\sharp}$-scheme. We refer to $(Z, \mathcal{M})_{\operatorname{crys}(n)}:=\left((Z, \mathcal{M}) / S_{n}^{\sharp}\right)_{\text {crys }},(Z, \mathcal{M})_{\text {crys }}:=\left((Z, \mathcal{M}) / S^{\sharp}\right)_{\text {crys }}$ as the absolute crystalline sites. One has the absolute log crystalline complexes $R u_{Z / \mathbb{Z}_{p} *}^{\log }\left(\mathcal{O}_{Z / \mathbb{Z}_{p}}\right), R \Gamma_{\text {crys }}(Z, \mathcal{M})_{(n)}:=R \Gamma\left((Z, \mathcal{M})_{\operatorname{crys}(n)}, \mathcal{O}_{Z /\left(\mathbb{Z} / p^{n}\right)}\right)$, and $R \Gamma_{\text {crys }}(Z, \mathcal{M}):=R \Gamma\left((Z, \mathcal{M})_{\text {crys }}, \mathcal{O}_{Z / \mathbb{Z}_{p}}\right)=R \Gamma\left(Z_{\text {ét }}, R u_{Z / \mathbb{Z}_{p} *}^{\log }\left(\mathcal{O}_{Z / \mathbb{Z}_{p}}\right)\right)=$ $\operatorname{holim}_{n} R \Gamma_{\text {crys }}(Z, \mathcal{M})_{(n)}$. We denote by $H_{\text {crys }}^{i}(Z, \mathcal{M})$ the absolute crystalline cohomology $H^{i} R \Gamma_{\text {crys }}(Z, \mathcal{M})$.

If $(Z, \mathcal{M})$ is an integral quasi-coherent $\log \mathbb{Z}_{p^{-}}$-scheme, then for $n \geq 1$ we set $(Z, \mathcal{M})_{n}:=\left(Z_{n}, \mathcal{M}_{n}\right)$, where $Z_{n}:=Z \otimes \mathbb{Z} / p^{n}$ and $\mathcal{M}_{n}$ is the restriction of $\mathcal{M}$ to $Z_{n} \subset Z$. We set $R \Gamma_{\text {crys }}(Z, \mathcal{M}):=R \Gamma_{\text {crys }}\left(Z_{1}, \mathcal{M}_{1}\right)$, etc.

Remarks. (i) If $Z$ is a flat $\mathbb{Z}_{p}$-scheme, then $(Z, \mathcal{M})_{n}$ is a $\log S^{\sharp}$-scheme, and its log crystalline complex equals $R \Gamma_{\text {crys }}(Z, \mathcal{M})$ by the crystalline invariance property. 
(ii) For a perfect field $k$ of characteristic $p$, let $S_{k n}^{\sharp}$ be $\operatorname{Spec} W_{n}(k)$ equipped with the trivial $\log$ structure and $\mathcal{I}=p W_{n}(k)$ with the standard pd structure. If $(Z, \mathcal{M})$ is an integral quasi-coherent $\log k$-scheme $(Z, \mathcal{M})$, then every its pd-thickening is automatically a $W(k)$-scheme, i.e., $\left((Z, \mathcal{M}) / S_{k}^{\sharp}\right)_{\text {crys }}=$ $(Z, \mathcal{M})_{\text {crys. }}$ Thus the absolute crystalline complexes of $(Z, \mathcal{M})$ are $W(k)$ algebras, etc.

1.13. A digression on difference equations and Dwork's trick. Let $R$ be a ring, $\varphi_{R}$ be an endomorphism of $R$. Denote by $R_{\varphi}$ the associative algebra generated by its subring $R$ and $\varphi$ with relation $\varphi r=\varphi_{R}(r) \varphi, r \in R$. Thus a (left) $R_{\varphi}$-module is the same as a left $R$-module $F$ equipped with a $\varphi_{R}$-semilinear endomorphism $\varphi_{F}$ called $\varphi_{R}$-action or simply $\varphi$-action; we usually abbreviate $\left(F, \varphi_{F}\right)$ to $F$. Denote by $R_{\varphi}$-mod the abelian category of $R_{\varphi}$-modules and by $D_{\varphi}(R)$ its derived category.

For $F_{1}, F_{2} \in R_{\varphi}$-mod consider the map $\delta: \operatorname{Hom}_{R}\left(F_{1}, F_{2}\right) \rightarrow \operatorname{Hom}_{R}^{(\varphi)}\left(F_{1}\right.$, $\left.F_{2}\right), \delta=\delta^{\prime}-\delta^{\prime \prime}, \delta^{\prime}(\xi):=\xi \varphi_{F_{1}}, \delta^{\prime \prime}(\xi):=\varphi_{F_{2}} \xi$, where $\operatorname{Hom}_{R}^{(\varphi)}$ is the group of $\varphi$-semilinear maps, so $\operatorname{Ker}(\delta)=\operatorname{Hom}_{R_{\varphi}}\left(F_{1}, F_{2}\right)$. Set $\operatorname{Hom}_{R_{\varphi}}^{\natural}\left(F_{1}, F_{2}\right):=$ Cone $(\delta)[-1]$.

Exercises. (i) The map $R \operatorname{Hom}_{R_{\varphi}}\left(F_{1}, F_{2}\right) \rightarrow R \operatorname{Hom}_{R_{\varphi}}^{\natural}\left(F_{1}, F_{2}\right)$ is a quasiisomorphism, and $R \operatorname{Hom}_{R_{\varphi}}^{\natural}\left(F_{1}, F_{2}\right)$ equals $\operatorname{Cone}\left(R \operatorname{Hom}_{R}\left(F_{1}, F_{2}\right) \rightarrow\right.$ $\left.R \operatorname{Hom}_{R}^{(\varphi)}\left(F_{1}, F_{2}\right)\right)[-1]$ where $R \operatorname{Hom}_{R}, R \operatorname{Hom}_{R}^{(\varphi)}$ are computed in the derived category of $R$-modules. ${ }^{30}$

(ii) One has $\operatorname{Hom}_{R_{\varphi}}^{\natural}\left(F_{1}, F_{2}\right)=\operatorname{Hom}_{R_{\varphi}}\left(F_{1}^{l}, F_{2}\right)=\operatorname{Hom}_{R_{\varphi}}\left(F_{1}, F_{2}^{r}\right)$ where $F_{1}^{l}$, $F_{2}^{r}$ are natural left and right two-term resolutions of $F_{1}, F_{2}$. Explicitly, $F_{1}^{l 0}=R_{\varphi} \otimes_{R} F_{1}=\oplus_{n \geq 0} \varphi_{R}^{n *} F_{1}$, where $\varphi_{R}^{n *} F_{1}:=R \otimes_{\varphi^{n}} F_{1}, F_{1}^{l-1}=\left(\varphi_{R}^{*} F_{1}\right)^{l 0}$ (so if $F_{1}$ is $R$-projective, then the resolution $F_{1}^{l}$ is $R_{\varphi}$-projective), and $F_{2}^{r 0}=\Pi_{n \geq 0} \phi_{R *}^{n} F_{2}, F_{2}^{r 1}=\left(\varphi_{R *} F_{2}\right)^{r 0}$.

An $R_{\varphi}$-complex is said to be $R$-perfect if it is perfect as a complex of $R$-modules; such objects form a thick subcategory $D_{\varphi}^{\text {perf }}(R)$ of $D\left(R_{\varphi}\right)$. Let $R_{\varphi}^{\mathrm{prf}}$-mod be the category of $R_{\varphi}$-modules which are finitely generated and projective as $R$-modules. An $R_{\varphi}$-module $M$ is said to be nondegenerate if the $R$-linear extension $\varphi_{M}^{l}: \varphi_{R}^{*} M \rightarrow M$ of $\varphi_{M}$ is an isogeny, i.e., $\varphi_{R}^{*} M \otimes \mathbb{Q} \stackrel{\sim}{\rightarrow}$ $M \otimes \mathbb{Q}$; an $R_{\varphi}$-complex $C$ is nondegenerate if the composition $L \varphi_{R}^{*} C \otimes \mathbb{Q} \rightarrow$ $\varphi_{R}^{*} C \otimes \mathbb{Q} \rightarrow C \otimes \mathbb{Q}$ is a quasi-isomorphism. Denote by ? ${ }^{\text {nd }}$ the subcategory of ? formed by nondegenerate objects, e.g. we have a triangulated category $D_{\varphi}^{\text {perf }}(R)^{\text {nd }}$.

\footnotetext{
${ }^{30}$ Hint: $\delta$ is surjective when $F_{1}$ is a free $R_{\varphi}$-module, and $R_{\varphi}$ is free as a (left) $R$-module.
} 
Lemma. (i) $R_{\varphi}^{\text {prf }}$-mod generates $D_{\varphi}^{\text {perf }}(R)$ as a triangulated category. (ii) If $R$ is a mixed characteristic dvr, then $R_{\varphi}^{\text {prf }}-\bmod ^{\text {nd }}$ generates $D_{\varphi}^{\text {perf }}(R)^{\text {nd }}$.

Proof. (i) Let $C$ be an $R$-perfect $R_{\varphi}$-complex. As an $R$-complex, it is quasiisomorphic to a complex of finitely generated projective $R$-modules of finite amplitude $[a, b], a \geq b$. We show that $C$ lies in the triangulated subcategory $D_{\varphi}^{\text {prf }}(R)$ of $D_{\varphi}^{\text {perf }}(R)$ generated by $R_{\varphi}^{\text {prf }}$-mod using induction by $a-b$.

If $a=b$, then $C \stackrel{\sim}{\rightarrow}\left(H^{a} C\right)[-a], H^{a} C \in R_{\varphi}^{\text {prf }}$-mod, and we are done. Suppose $n=a-b \geq 1$. Then $H^{a} C$ is a finitely generated $R$-module. Pick a surjection $P \rightarrow H^{a} C$ where $P$ is a finitely generated projective $R$-module. The $\varphi$-action on $H^{a} C$ can be lifted to $P$, i.e., there is a $\varphi$-action $\varphi_{P}$ on $P$ such that $P \rightarrow H^{a} C$ is a map of $R_{\varphi}$-modules. Consider the projective $R_{\varphi}$-resolution $P^{l}$ of $P=\left(P, \varphi_{P}\right)$. The above surjection lifts to a map of $R_{\varphi}$-complexes $g: P^{l}[-a] \rightarrow C$. Since $P^{l}$ is quasi-isomorphic to $P, C$ lies in $D_{\varphi}^{\text {prf }}(R)$ if (and only if) $\mathcal{C}$ one $(g)$ lies in $D_{\varphi}^{\text {prf }}(R)$, and the latter assertion is true by the induction assumption, q.e.d.

(ii) To make the proof of (i) work in the present situation, it suffices to check that for $C$ nondegenerate and any $P \rightarrow H^{a} C$ as in loc. cit., one can choose $\varphi_{P}$ so that $\left(P, \varphi_{P}\right)$ is nondegenerate. Let $\varphi_{P}$ be any lifting of the $\varphi$-action on $H^{a} C$. Let $Q \subset P$ be the kernel of the map $P \rightarrow H^{a} C \otimes \mathbb{Q}$. Then $\varphi_{P}$ preserves $Q, P / Q$ is a projective $R$-module, and $(P / Q) \otimes \mathbb{Q} \stackrel{\sim}{\rightarrow} H^{a} C \otimes \mathbb{Q}$, so $\left(P / Q, \varphi_{P / Q}\right) \in R_{\varphi}^{\text {prf }}$-mod ${ }^{\text {nd }}$. We can modify $\varphi_{P}$ by adding to it any map $\varphi_{R}^{*} P \rightarrow Q \subset P$ which is sufficiently small in $p$-adic topology (here $p$ is the residual characteristic of $R$ ). If this map is sufficiently general, then the resulting $\varphi_{Q}$, hence $\varphi_{P}$, is an isogeny; we are done.

Suppose now $R$ is a $p$-adically complete commutative algebra, $R \stackrel{\sim}{\rightarrow}$ $\lim R / p^{n} R$, and $I \subset R$ is a closed (for the $p$-adic topology) ideal preserved by $\varphi_{R}$ such that the induced endomorphism $\varphi_{W}$ of $W:=R / I$ is invertible and $\varphi_{R}$ is topologically nilpotent on $I$ (i.e., $\varphi_{R}$ acts nilpotently on $I / p I$, hence on $\left.I / p^{n} I\right)$. Then $I$ consists of all $r \in R$ such that $\varphi_{R}^{n}(r) \rightarrow 0$. The projection $R \rightarrow W$ admits a unique section $s: W \rightarrow R$ compatible with the action of $\varphi$ (which is automatically a ring homomorphism). ${ }^{31}$ The corresponding base change functors $\pi^{*}: W_{\varphi^{-} \bmod } \rightleftarrows R_{\varphi^{-}} \bmod : i^{*}, \pi^{*} M:=R \otimes_{W} M$, $i^{*} N:=W \otimes_{R} N=N / I N, i^{*} \pi^{*} M=M$, preserve the subcategories ?prf mod; the derived functors $L \pi^{*}, L i^{*}$ preserve the subcategories $D_{\varphi}^{\text {perf }}(?)$. They preserve the subcategories of non-degenerate objects as well.

${ }^{31}$ To see this, notice that for every (set-theoretic) section $s^{\prime}: W \rightarrow R$ the sequence of sections $\varphi_{R}^{n} s^{\prime} \varphi_{W}^{-n}$ converges $p$-adically, and its limit $s$ does not depend on the choice of $s^{\prime}$. 
We say that $\varphi$ is strongly topologically nilpotent on $I$ if for every $m>0$ one can find a finite filtration of $I$ by closed $\varphi_{R}$-invariant ideals such that $\varphi_{R}(\operatorname{gr} \cdot I) \subset p^{m} \operatorname{gr} I$.

Proposition. If $W$ is a mixed characteristic dvr and $\varphi$ is strongly topologically nilpotent on $I$, then the functors $L \pi^{*}, L i^{*}$ yield mutually inverse equivalences

$$
D_{\varphi}^{\text {perf }}(W)^{n d} \otimes \mathbb{Q} \stackrel{\sim}{\leftrightarrow} D_{\varphi}^{\text {perf }}(R)^{n d} \otimes \mathbb{Q} .
$$

Proof. (a) Let us show that for any $P \in W^{\text {prf }}{ }_{-}$mod $^{\text {nd }}$ and $Q \in R^{\text {prf }}$-mod, one has $\operatorname{RHom}_{W_{\varphi}}(P, I Q) \otimes \mathbb{Q}=0$.

For any $M \in W_{\varphi}$-mod consider the two-term complex $\mathcal{C}(M):=$ $\operatorname{Hom}_{W_{\varphi}}^{\natural}(P, M), \mathcal{C}^{0}(M)=\operatorname{Hom}_{W}(P, M), \mathcal{C}^{1}(M)=\operatorname{Hom}_{W}\left(\varphi_{W}^{*} P, M\right)$. By Exercises, $R \operatorname{Hom}_{W_{\varphi}}(P, M)=\mathcal{C} \cdot(M)$. Let $\mathcal{C}_{\star}(M)$ be the complex with the same components as $\mathcal{C}^{\cdot}(M)$ and the differential $\delta^{\prime}(\xi)=\xi \varphi_{P}^{l}$. Since $P$ is nondegenerate, $\mathcal{C}_{\star}(M) \otimes \mathbb{Q}$ is acyclic.

The functor $\mathcal{C}$ is exact. We prove that $\mathcal{C}(I Q) \otimes \mathbb{Q}$ is acyclic by defining a finite filtration $I Q$ on $I Q$ such that for $F:=\operatorname{gr} I Q$ one has $\mathcal{C} \cdot(F) \simeq \mathcal{C}_{\star}^{*}(F)$.

For $m$ sufficiently large, there is $\psi: P \rightarrow \varphi_{W}^{*} P$ with $\varphi_{P}^{l} \psi=p^{m-1} \operatorname{id}_{P}$, $\psi \varphi_{P}^{l}=p^{m-1} \operatorname{id}_{\varphi_{W}^{*} P}$. By the condition of the proposition, one can find a finite filtration $I^{(\cdot)}$ on $I$ by closed $\varphi$-invariant ideals such that $\varphi_{R}\left(\mathrm{gr}^{(\cdot)} I\right) \subset$ $p^{m} \mathrm{gr}^{(\cdot)} I$. Set $I Q:=I^{(\cdot)} Q$.

One has $\varphi_{F}(F) \subset p^{m} F$. Therefore $\delta^{\prime \prime}$ on $\mathcal{C}(F)$ is divisible by $p^{m}$. Set $\chi:=$ $\psi^{\tau}\left(p^{-m} \delta^{\prime \prime}\right) \in \operatorname{End}\left(\mathcal{C}^{0}(F)\right)$; then $\delta^{\prime \prime}=p \delta^{\prime} \chi$, i.e., $\delta(1-p \chi)=\delta^{\prime}$. Since $\mathcal{C}^{0}(F)$ is p-adically complete, $1-p \chi$ is invertible, so it yields $\mathcal{C}_{\star}(F) \stackrel{\sim}{\rightarrow} \mathcal{C} \cdot(F)$, q.e.d.

(b) The lemma and (a) imply that for every $P \in D_{\varphi}^{\text {perf }}(W)^{\text {nd }}$ and $Q \in$ $D_{\varphi}^{\text {perf }}(R)$ one has $R \operatorname{Hom}_{R_{\varphi}}\left(L \pi^{*} P, Q\right) \otimes \mathbb{Q} \stackrel{\sim}{\rightarrow} R \operatorname{Hom}_{W_{\varphi}}\left(P, L i^{*} Q\right) \otimes \mathbb{Q}$. Thus the two functors $L \pi^{*}: D_{\varphi}^{\text {perf }}(W)^{\text {nd }} \otimes \mathbb{Q} \leftrightarrows D_{\varphi}^{\text {perf }}(R)^{\text {nd }} \otimes \mathbb{Q}: L i^{*}$ are adjoint.

Since $L i^{*}$ is left inverse to $L \pi^{*}$, it remains to show that for $Q \in D_{\varphi}^{\text {perf }}(R)^{\text {nd }}$ the adjunction $L \pi^{*} L i^{*}(Q \otimes \mathbb{Q}) \rightarrow Q \otimes \mathbb{Q}$ is a quasi-isomorphism. We can replace $Q \otimes \mathbb{Q}$ by $\mathcal{C}$ one $\left(L \pi^{*} L i^{*}(Q \otimes \mathbb{Q}) \rightarrow Q \otimes \mathbb{Q}\right)$, so it suffices to check that $L i^{*}(Q \otimes \mathbb{Q})=0$ implies $Q \otimes \mathbb{Q}=0$. Since $Q$ is nondegenerate, the support $S$ of $Q \otimes \mathbb{Q}$, which is a closed subset of $\operatorname{Spec}(R \otimes \mathbb{Q})$, is $\varphi_{R}$-invariant. If $S$ is non-empty, then this implies that $\operatorname{Spec}(W \otimes \mathbb{Q})$ lies in $S .^{32}$ Since $Q \otimes \mathbb{Q}$ is perfect, this contradicts the assumption $L i^{*}(Q \otimes \mathbb{Q})=0$, q.e.d.

\footnotetext{
${ }^{32}$ Indeed, otherwise there is $r \in R \backslash I$ that vanishes on $S$. Suppose $r \bmod I \in$ $p^{n} W^{\times}$. Since $\varphi_{R}$ is topologically nilpotent on $I$ and invertible on $W$, for $m \gg 0$ one has $\varphi_{R}^{m *}(r)=p^{n}\left(a_{m}+p b_{m}\right)$, where $a_{m} \in s\left(W^{\times}\right)$and $b_{m} \in I$. Hence $\varphi_{R}^{m *}(r) \in p^{n} R^{\times}$ since $R$ is $p$-adically complete. Since $S$ is $\varphi_{R}$-invariant, $\varphi_{R}^{m *}(r)$ vanishes on $S$, i.e., $S=\emptyset$.
} 
1.14. Frobenius crystals. Suppose $S^{\sharp}$ as in the beginning of 1.12 is equipped with an endomorphism $\varphi_{S^{\sharp}}$ whose restriction to $\left(S_{1}, \mathcal{L}_{1}\right)$ is the Frobenius map $\operatorname{Fr}_{\left(S_{1}, \mathcal{L}_{1}\right)}$. Then for any integral quasi-coherent $(Y, \mathcal{N})$ over $S_{1}^{\sharp}$ the endomorphism $\varphi=\left(F r_{(Y, \mathcal{N})}, \varphi_{S^{\sharp}}\right)$ of $(Y, \mathcal{N}) / S^{\sharp}$ acts on $(Y / S)_{\text {crys. A Frobenius }}^{\log }$ $\mathcal{O}_{Y / S}$-module is a pair $\left(\mathcal{F}, \varphi_{\mathcal{F}}\right)$, where $\mathcal{F}$ is an $\mathcal{O}_{Y / S}$-module on $(Y / S)_{\text {crys }}^{\text {log }}$, $\varphi_{\mathcal{F}}$ is a $\varphi_{\text {crys }}$-action on $\mathcal{F}$, i.e., a map $\varphi_{\mathcal{F}}: \mathcal{F} \rightarrow \varphi_{\text {crys* }}(\mathcal{F})$; if $\mathcal{F}$ is an $\mathcal{O}_{Y / S^{-}}$-crystal, we call $\left(\mathcal{F}, \varphi_{\mathcal{F}}\right)$ an $F$-crystal. Frobenius $\mathcal{O}_{Y / S^{-}}$-modules form an abelian category; let $D_{\varphi}\left((Y / S)_{\text {crys }}^{\log }, \mathcal{O}_{Y / S}\right)$ be its derived category, and $D_{\varphi}^{\mathrm{pcr}}(Y / S)=D_{\varphi}^{\mathrm{pcr}}\left((Y, \mathcal{N}) / S^{\sharp}\right)$ be the thick subcategory of perfect $F$-crystals, i.e., those $\left(\mathcal{F}, \varphi_{\mathcal{F}}\right)$ that $\mathcal{F}$ is a perfect crystal (see 1.11). Such an $\left(\mathcal{F}, \varphi_{\mathcal{F}}\right)$ is nondegenerate if the map $L \varphi_{\text {crys }}^{*}(\mathcal{F}) \rightarrow \mathcal{F}$ that comes from $\varphi_{\mathcal{F}}$ is an isogeny, i.e., it is a quasi-isomorphism in $D^{\text {pcr }}(Y / S) \otimes \mathbb{Q}$; the corresponding category is denoted by $D_{\varphi}^{\mathrm{pcr}}(Y / S)^{\mathrm{nd}}$.

A morphism $\theta:\left(Y^{\nu}, \mathcal{N}^{\nu}\right) / S^{\nu \sharp} \rightarrow(Y, \mathcal{N}) / S^{\sharp}$ compatible with $\varphi_{S^{\nu \sharp}}, \varphi_{S^{\sharp}}$ yields the pullback functor $\theta_{\text {crys }}^{*}$ between the categories of Frobenius $\mathcal{O}$ modules. The derived functor $L \theta_{\text {crys }}^{*}$ preserves the subcategories of (nondegenerate) perfect $F$-crystals, and it is compatible with $L \theta_{\text {crys }}^{*}$ from 1.5 via the forgetful functor $\left(\mathcal{F}, \varphi_{\mathcal{F}}\right) \mapsto \mathcal{F}$.

Remarks. (i) The endofunctor $L \varphi_{\text {crys }}^{*}$ of $D_{\varphi}^{\mathrm{pcr}}(Y / S)^{\mathrm{nd}} \otimes \mathbb{Q}$ is canonically isomorphic to the identity functor.

(ii) For a perfect F-crystal $\left(\mathcal{F}, \varphi_{\mathcal{F}}\right)$, its nondegeneracy is a $Y_{\text {ét-local prop- }}$ erty. Suppose $Y$ is affine and $P=\left(P, \mathcal{N}_{P}\right)$ is its pd- $S^{\sharp}$-smooth formal thickening (i.e., $P_{n}$ are pd- $S_{n}^{\sharp}$-smooth thickenings of $Y$ ) equipped with a lifting $\varphi_{P}$ of $\varphi_{S^{\sharp}}$. Write $P=\operatorname{Spf} R, R$ is a $p$-adically complete ring, so $P_{n}=\operatorname{Spec} R_{n}$ and we have endomorphism $\varphi_{R}=\varphi_{P}^{*}$ of $R$. Then $\mathcal{F}(P):=$ $\operatorname{holim}_{n} \mathcal{F}\left(P_{n}\right)$ is a perfect $R$-complex equipped with a $\varphi_{R}$-action $\varphi_{\mathcal{F}(P)}$. Let $\varphi_{\mathcal{F}(P)}^{l}: R \otimes_{R, \varphi_{R}}^{L} \mathcal{F}(P)=\operatorname{holim}_{n} R_{n} \otimes_{R_{n}, \varphi_{R_{n}}}^{L} \mathcal{F}\left(P_{n}\right) \rightarrow \mathcal{F}(P)$ be its $R$-linear extension. Then $\mathcal{F}$ is nondegenerate if and only if $\varphi_{\mathcal{F}(P)}^{l} \otimes \mathbb{Q}$ is a quasiisomorphism.

We usually abbreviate $\left(\mathcal{F}, \varphi_{\mathcal{F}}\right)$ to $\mathcal{F}$, and denote by $\operatorname{Hom}_{\varphi}\left(\mathcal{F}_{1}, \mathcal{F}_{2}\right)$ the group of Frobenius $\mathcal{O}_{Y / S^{-}}$module morphisms. One has an exact sequence $0 \rightarrow \operatorname{Hom}_{\varphi}\left(\mathcal{F}_{1}, \mathcal{F}_{2}\right) \rightarrow \operatorname{Hom}\left(\mathcal{F}_{1}, \mathcal{F}_{2}\right) \rightarrow \operatorname{Hom}\left(\mathcal{F}_{1}, \varphi_{\text {crys* }}\left(\mathcal{F}_{2}\right)\right)$, the last arrow is $\xi \mapsto \varphi_{\mathcal{F}_{2}} \xi-\varphi_{\text {crys* }}(\xi) \varphi_{\mathcal{F}_{1}}$.

Lemma. For any $\mathcal{F}_{1}, \mathcal{F}_{2} \in D_{\varphi}^{+}\left((Y / S)_{\text {crys }}^{\log }, \mathcal{O}_{Y / S}\right)$ one has a canonical quasiisomorphism $\operatorname{RHom}_{\varphi}\left(\mathcal{F}_{1}, \mathcal{F}_{2}\right) \stackrel{\sim}{\rightarrow} \operatorname{Cone}\left(\operatorname{RHom}\left(\mathcal{F}_{1}, \mathcal{F}_{2}\right) \rightarrow \operatorname{RHom}\left(\mathcal{F}_{1}\right.\right.$, $\left.\left.R \varphi_{\text {crys* }}\left(\mathcal{F}_{2}\right)\right)\right)[-1]$. 
Proof. The above exact sequence yields a map of complexes $\alpha: R \operatorname{Hom}_{\varphi}\left(\mathcal{F}_{1}\right.$, $\left.\mathcal{F}_{2}\right) \rightarrow \operatorname{Cone}\left(R \operatorname{Hom}\left(\mathcal{F}_{1}, \mathcal{F}_{2}\right) \rightarrow R \operatorname{Hom}\left(\mathcal{F}_{1}, R \varphi_{\text {crys* }}\left(\mathcal{F}_{2}\right)\right)\right)[-1]$. Let us check that $\alpha$ is a quasi-isomorphism.

The forgetful functor $\left(\mathcal{F}, \varphi_{\mathcal{F}}\right) \mapsto \mathcal{F}$ from Frobenius $\mathcal{O}_{Y / S}$-modules to $\mathcal{O}_{Y / S}$-modules, admits a right adjoint $\mathcal{F} \mapsto \mathcal{F}_{(\varphi)}$. Explicitly, $\mathcal{F}_{(\varphi)}=$ $\Pi_{n \geq 0} \varphi_{\text {crys* }}^{n}(\mathcal{F})$ and $\varphi_{\mathcal{F}_{(\varphi)}}$ is the projection $\Pi_{n \geq 0} \varphi_{\text {crys* }}^{n}(\mathcal{F}) \rightarrow \Pi_{n \geq 1} \varphi_{\text {crys* }}^{n}(\mathcal{F})$. The functor $\mathcal{F} \mapsto \mathcal{F}_{(\varphi)}$ is left exact and sends injective objects to injective ones. Thus every Frobenius $\mathcal{O}_{Y / S}$-module admits an embedding into $\mathcal{G}_{(\varphi)}$ where $\mathcal{G}$ is some injective $\mathcal{O}_{Y / S}$-module. Therefore it suffices to check that $\alpha$ is a quasi-isomorphism assuming that $\mathcal{F}_{1}=\mathcal{F}$ is any Frobenius $\mathcal{O}_{Y / S}$-module and $\mathcal{F}_{2}=\mathcal{G}_{(\varphi)}$ with $\mathcal{G}$ injective. Then $R \operatorname{Hom}_{\varphi}\left(\mathcal{F}, \mathcal{G}_{(\varphi)}\right) \tilde{\leftarrow} \operatorname{Hom}(\mathcal{F}, \mathcal{G}) \stackrel{\sim}{\rightarrow}$ Cone $\left(\operatorname{Hom}\left(\mathcal{F}, \mathcal{G}_{(\varphi)}\right) \rightarrow \operatorname{Hom}\left(\mathcal{F}, \varphi_{\text {crys* }}\left(\mathcal{G}_{(\varphi)}\right)\right)[-1] \stackrel{\sim}{\rightarrow} \mathcal{C}\right.$ one $\left(R \operatorname{Hom}\left(\mathcal{F}, \mathcal{G}_{(\varphi)}\right) \rightarrow\right.$ $\left.R \operatorname{Hom}\left(\mathcal{F}, R \varphi_{\text {crys* }}\left(\mathcal{G}_{(\varphi)}\right)\right)\right)[-1]$, q.e.d.

Suppose now our $(Y, \mathcal{N})$ is a fine $\log$ scheme, $Y$ is affine, and there is $P$ as in Remark (ii) such that $R$ has no $p$-torsion. Let $f:(Z, \mathcal{M}) \rightarrow(Y, \mathcal{N})$ be a $\log$ smooth map of Cartier type with $(Z, \mathcal{M})$ fine and $Z$ is proper over $Y$. Consider $\mathcal{F}:=R f_{\text {crys* }}\left(\mathcal{O}_{Z / S}\right)$. By the theorem in $1.11, \mathcal{F} \in D_{\varphi}^{\text {pcr }}(Y / S)$. The next is a $\log$ version [HK] 2.24 of a theorem of Berthelot-Ogus [BO2] 1.3:

Theorem. The perfect $F$-crystal $\mathcal{F}$ is nondegenerate.

Proof. We use notation from Remark (ii). Set $C:=\mathcal{F}(P)=R \Gamma\left((Z / P)_{\text {crys }}^{\log \text {, }}\right.$ $\left.\mathcal{O}_{Z / P}\right)$, so $C_{n}:=C \otimes \mathbb{Z} / p^{n}=\mathcal{F}\left(P_{n}\right)=R \Gamma\left(\left(Z / P_{n}\right)_{\text {crys }}^{\log }, \mathcal{O}_{Z / P_{n}}\right), C=\operatorname{holim}_{n} C_{n}$. Then $C$ carries the Frobenius endomorphism $\varphi_{C}=\varphi_{\mathcal{F}(P)}$, and we want to prove that $\varphi_{C}^{l} \otimes \mathbb{Q}$ is a quasi-isomorphism.

Set $\mathcal{G}_{n}:=R u_{Z / P_{n} *}^{\log }\left(\mathcal{O}_{Z / P_{n}}\right), \mathcal{G}:=R u_{Z / P *}^{\log }\left(\mathcal{O}_{Z / P}\right)=\operatorname{holim}_{n} \mathcal{G}_{n}$. These are $R_{n^{-}}$and $R$-complexes of sheaves on $Z_{\text {ét }}$, and (i) of the theorem in 1.11 implies that $\mathcal{G}_{n}=\mathcal{G} \otimes^{L} \mathbb{Z} / p^{n}$. They carry natural Frobenius $\varphi_{R^{-}}$ actions $\varphi_{\mathcal{G}_{n}}, \varphi_{\mathcal{G}}$. One has $C_{n}=R \Gamma\left(Z_{\text {ét }}, \mathcal{G}_{n}\right), C=R \Gamma\left(Z_{\text {ét }}, \mathcal{G}\right)$, and $\varphi_{C}$ comes from $\varphi_{\mathcal{G}}$. Let $\varphi_{\mathcal{G}_{n}}^{l}: R_{n} \otimes_{R_{n}, \varphi_{R_{n}}}^{L} \mathcal{G}_{n} \rightarrow \mathcal{G}_{n}$ be the $R_{n}$-linear extensions of $\varphi_{\mathcal{G}_{n}}$; set $\varphi_{\mathcal{G}}^{l}:=\operatorname{holim}_{n} \varphi_{\mathcal{G}_{n}}^{l}: R \widehat{\otimes}_{R, \varphi_{R}}^{L} \mathcal{G}:=\operatorname{holim}_{n}\left(R_{n} \otimes_{R_{n}, \varphi_{R_{n}}}^{L} \mathcal{G}_{n}\right) \rightarrow \mathcal{G}$. Since $R \Gamma\left(Z_{\text {ét }}, R \widehat{\otimes}_{R, \varphi_{R}}^{L} \mathcal{G}\right)=\operatorname{holim}_{n}\left(R_{n} \otimes_{R_{n}, \varphi_{R_{n}}}^{L} C_{n}\right)$ and $R \Gamma\left(Z_{\text {ét }}, \cdot \otimes \mathbb{Q}\right)=$ $R \Gamma\left(Z_{\text {ét }}, \cdot\right) \otimes \mathbb{Q}$ (for $Z$ is quasi-compact quasi-separated), it suffices to show that $\varphi_{\mathcal{G}}^{l} \otimes \mathbb{Q}:\left(R \widehat{\otimes}_{R, \varphi_{R}}^{L} \mathcal{G}\right) \otimes \mathbb{Q} \rightarrow \mathcal{G} \otimes \mathbb{Q}$ is a quasi-isomorphism.

Now our assertion is $Z$-local, so to check it we can assume that $Z$ is affine. Choose a $\log$ smooth (formal) lifting $\left(T, \mathcal{M}_{T}\right) /\left(P, \mathcal{N}_{P}\right)$ of $(Z, \mathcal{M}) /(Y, \mathcal{N})$ together with a lifting $\varphi_{T}$ of the Frobenius compatible with $\varphi_{P}$. By (1.8.1), one has $\left(\mathcal{G}, \varphi_{\mathcal{G}}\right)=\left(\Omega, \varphi_{T}^{*}\right)$ where $\Omega=\varliminf^{\prime} \Omega_{n}, \Omega_{n}:=\Omega_{\left(T, \mathcal{M}_{T}\right)_{n} /\left(P . \mathcal{N}_{P}\right)_{n}}$. Consider the $p$-adic filtration on $\Omega$; let $\Phi^{*}$ be its shift, i.e., $\Phi^{m} \Omega^{*}$ is the 
maximal subcomplex of $\Omega$ such that $\Phi^{m} \Omega^{n} \subset p^{m} \Omega^{n}$. Since $\varphi_{T}^{*}\left(\Omega^{i}\right) \subset p^{i} \Omega^{i}$, one has $\varphi_{T}^{*}\left(\Omega^{*}\right) \subset \Phi^{0} \Omega^{*}$. Since $\left(\Omega^{*} / \Phi^{0} \Omega^{*}\right) \otimes \mathbb{Q}=0$ and $\Omega^{i}$ are $R$-flat, to finish the proof it suffices to check that the $R$-linear map $\varphi_{T}^{* l}: R \widehat{\otimes}_{R, \varphi_{R}} \Omega \rightarrow \Phi^{0} \Omega$. is a quasi-isomorphism.

Our complexes of sheaves are $p$-adically complete, have no $p$-torsion, and $\Phi^{m} \Omega=p^{m} \Phi^{0} \Omega$ for $m \geq 0$. Thus it suffices to show that $\varphi_{T_{1}}^{* l}$ : $R_{1} \otimes_{R_{1}, \varphi_{R_{1}}} \Omega_{1} \rightarrow \operatorname{gr}_{\Phi}^{0} \Omega$ is a quasi-isomorphism. Let $\overline{g r}_{\Phi}^{0} \Omega$ be the quotient of $\operatorname{gr}_{\Phi}^{0} \Omega$ modulo the subcomplex generated by the images of $p^{i+1} \Omega^{i} \subset \Phi^{0} \Omega^{i}$. This subcomplex is acyclic, and the multiplication by $p^{-i}$ yields an isomorphism $\overline{g r}_{\Phi}^{0} \Omega^{i} \stackrel{\sim}{\rightarrow} H^{i} \Omega_{1}$. The composition $R_{1} \otimes_{R_{1}, \varphi_{R_{1}}} \Omega_{1} \rightarrow \operatorname{gr}_{\Phi}^{0} \Omega \rightarrow \overline{g r}_{\Phi}^{0} \Omega$ is an isomorphism: indeed, its components coincide, via the previous identification, with the Cartier isomorphism $C^{-1}$ from (1.10.2). We are done.

Remark. In fact, according to [BO1] 8.20, $\varphi_{T}^{* l}: R \widehat{\otimes}_{R, \varphi_{R}} \Omega \stackrel{\sim}{\rightarrow} \Phi^{0} \Omega$ comes from a natural global quasi-isomorphism $R \widehat{\otimes}_{R, \varphi_{R}} \mathcal{G} \stackrel{\sim}{\rightarrow} \Phi^{0} \mathcal{G}$.

Exercise. Using $r$-iterated Cartier isomorphism, identify the $r$-th differential of the spectral sequence for the $p$-adic filtration on $\Omega$ with the de Rham differential.

1.15. $(\varphi, N)$-modules. Let $k$ be a perfect field of characteristic $p, W:=$ $W(k), K_{0}:=$ Frac $W, \varphi$ be the Frobenius automorphism of $W$ and $K_{0}$. As in [F2] 4.2, a $\varphi$-module over $K_{0}$ is a pair $(V, \varphi)$, where $V$ is a finite-dimensional $K_{0}$-vector space, $\varphi=\varphi_{V}$ is a $\varphi$-semilinear automorphism of $V$; a $(\varphi, N)$ module is a triple $(V, \varphi, N)$, where $(V, \varphi)$ is a $\varphi$-module and $N=N_{V}$ is a $K_{0^{-}}$ linear endomorphism of $V$ such that $N \varphi=p \varphi N$ (then $N$ is automatically nilpotent). One says that $V$ is effective (or of nonnegative slope) if it contains a $\varphi_{V}$-invariant $W$-lattice, hence one preserved by both $\varphi_{V}$ and $N_{V}$. The category $(\varphi, N)$-mod of $(\varphi, N)$-modules is naturally a Tannakian tensor $\mathbb{Q}_{p^{-}}$ category, and $(V, \varphi, N) \mapsto V$ is a fiber functor over $K_{0} \cdot{ }^{33}$ Let $(\varphi, N)^{\text {eff }}$-mod be its abelian tensor subcategory of effective modules, and $D_{\varphi, N}\left(K_{0}\right)^{\text {eff }} \subset$ $D_{\varphi, N}\left(K_{0}\right)$ be the corresponding bounded derived categories.

We usually abbreviate $(V, \varphi, N)$ to $V$. For $(\varphi, N)$-modules $V_{1}, V_{2}$, we denote by $\operatorname{Hom}_{\varphi, N}\left(V_{1}, V_{2}\right)$ the group of $(\varphi, N)$-module morphisms, and by $\operatorname{Hom}\left(V_{1}, V_{2}\right), \operatorname{Hom}^{(\varphi)}\left(V_{1}, V_{2}\right)$ the groups of $K_{0}$-linear and $\varphi$-semilinear maps. Let $\operatorname{Hom}_{\varphi, N}^{\natural}\left(V_{1}, V_{2}\right)$ be the complex $\operatorname{Hom}\left(V_{1}, V_{2}\right) \rightarrow \operatorname{Hom}^{(\varphi)}\left(V_{1}, V_{2}\right) \oplus$ $\operatorname{Hom}\left(V_{1}, V_{2}\right) \rightarrow \operatorname{Hom}^{(\varphi)}\left(V_{1}, V_{2}\right)$ supported in degrees [0,2] with the differential $d^{0}(\xi)=\left(\varphi_{2} \xi-\xi \varphi_{1}, N_{2} \xi-\xi N_{1}\right), d^{1}(\chi, \psi)=N_{2} \chi-p \chi N_{1}-p \varphi_{2} \psi+\psi \varphi_{1}$. Clearly $\operatorname{Hom}_{\varphi, N}\left(V_{1}, V_{2}\right)=H^{0} \operatorname{Hom}_{\varphi, N}^{\natural}\left(V_{1}, V_{2}\right)$.

\footnotetext{
${ }^{33}$ The same is true for the category of $\varphi$-modules.
} 
Exercise. Show that $R \operatorname{Hom}_{\varphi, N}\left(V_{1}, V_{2}\right) \stackrel{\sim}{\rightarrow} \operatorname{Hom}_{\varphi, N}^{\natural}\left(V_{1}, V_{2}\right)$.

Remark. Complexes $\operatorname{Hom}_{\varphi, N}^{\natural}$ compose naturally, so they provide a dg category structure on $(\varphi, N)$-modules. By Exercise, its homotopy category equals $D_{\varphi, N}\left(K_{0}\right)$.

Let $(Y, \mathcal{L})$ be an integral $\log k$-scheme such that $Y$ is a local scheme with residue field $k$, the maximal ideal of $\mathcal{O}_{Y}$ is killed by a power of Frobenius (say, $Y$ is Artinian), and $\mathcal{L} / \mathcal{O}_{Y}^{\times}=\mathbb{Z}_{\geq 0}$. We have the category $D_{\varphi}^{\mathrm{pcr}}(Y)$ of perfect F-crystals on the absolute crystalline site $(Y, \mathcal{L})_{\text {crys }}$ (see 1.12$)$.

Theorem. There is a canonical equivalence of triangulated categories

$$
\epsilon_{Y}: D_{\varphi, N}\left(K_{0}\right)^{e f f} \stackrel{\sim}{\rightarrow} D_{\varphi}^{p c r}(Y)^{n d} \otimes \mathbb{Q} .
$$

Proof. (a) Let $i^{0}:\left(Y^{0}, \mathcal{L}^{0}\right) \hookrightarrow(Y, \mathcal{L})$ be the exact embedding with $Y^{0}:=$ $Y_{\text {red }}=\operatorname{Spec} k$. Then $L i_{c r y s}^{0 *}: D_{\varphi}^{p c r}(Y)^{n d} \otimes \mathbb{Q} \rightarrow D_{\varphi}^{p c r}\left(Y^{0}\right)^{n d} \otimes \mathbb{Q}$ is an equivalence of categories. Indeed, since some power of $\operatorname{Fr}_{(Y, \mathcal{L})}$ factors through $i^{0}$, the claim follows from Remark (i) in 1.14. ${ }^{34}$ Our $\epsilon_{Y}$ will satisfy rigidity property (cf. [BO2] 2.1)

$$
\epsilon_{Y}=\left(L i_{\mathrm{crys}}^{0 *}\right)^{-1} \epsilon_{Y^{0}}
$$

Thus it suffices to construct $\epsilon_{Y^{0}}$. So for the rest of the proof we assume that $Y=Y^{0}$, i.e., $Y=\operatorname{Spec} k$.

(b) Let $\mathcal{L}^{g} \subset \mathcal{L}$ be the subset of generators of $\mathcal{L}$, i.e, the preimage of $1 \in \mathbb{Z}_{\geq 0}=\mathcal{L} / k^{\times}$; this is a $k^{\times}$-torsor. Let $W\left\langle t_{l}\right\rangle$ be the divided powers polynomial algebra generated by elements $t_{l}, l \in \mathcal{L}^{1}$, subject to relations $t_{a l}=[a] t_{l}$ for $a \in k^{\times}$; here $[a] \in W^{\times}$is the Teichmüller lifting of $a$. Let $R$ be the $p$-adic completion of $W\left\langle t_{l}\right\rangle, \mathcal{L}_{E}$ be the log structure on $E:=\operatorname{Spec} R$ generated by $t_{l}$. One has an exact embedding $i:(Y, \mathcal{L}) \hookrightarrow\left(E, \mathcal{L}_{E}\right), t_{l} \mapsto l$, and a $\varphi$-action $\varphi_{R}=\varphi$ on $\left(E, \mathcal{L}_{E}\right), \varphi_{R}^{*}\left(t_{l}\right)=t_{l}^{p}$, that extends the Frobenius endomorphism of $(Y, \mathcal{L})$. Then $\left(E, \mathcal{L}_{E}\right)_{n}$ (see 1.12) is a coordinate pd- $W_{n}(k)$ thickening of $(Y, \mathcal{L})$.

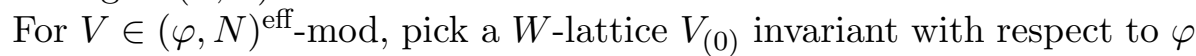
and $N$. The $R$-module $V_{(0) R}:=V_{(0)} \otimes_{W} R$ carries a log connection $\nabla$ and a $\varphi$-action $\varphi_{V}$ such that $\varphi_{V}$ and $\nabla_{t_{l} \partial_{t_{l}}}$ preserve generators $V_{(0)} \subset V_{(0) R}$ and coincide there with $\varphi$ and $N$. According to 1.7, the data of vector bundles $\left(V_{(0) R}\right)_{n}=V_{(0)} \otimes R / p^{n}$ on $E_{n}$ equipped with the above connection and $\varphi$ action yields an $\mathrm{F}$-crystal on $(Y, \mathcal{L})_{\text {crys }}$ which we denote by $\epsilon_{Y}\left(V_{(0)}\right)$. Thus

\footnotetext{
$L i_{\text {crys }}^{0 *}$.

${ }^{34}$ If $\operatorname{Fr}_{(Y, \mathcal{L})}^{m}=i^{0} h$, then $h i^{0}=F r_{\left(Y^{0}, \mathcal{L}^{0}\right)}^{m}$, so, by loc. cit., $L h_{\text {crys }}^{*}$ is inverse to
} 
a finite complex $V$ in $(\varphi, N)^{\text {eff }}$-mod equipped with a data of lattices $V_{(0)}$ invariant with respect to $\varphi, N$, and the differential, yields a nondegenerate perfect F-crystal $\epsilon_{Y}\left(V_{(0)}\right)$ on $(Y, \mathcal{L})_{\text {crys. }}$ Viewed up to isogeny, it does not depend on the choice of $V_{(0)}$. We have defined a triangulated functor $\epsilon_{Y}$ : $D_{\varphi, N}\left(K_{0}\right)^{\mathrm{eff}} \rightarrow D_{\varphi}^{\mathrm{pcr}}(Y)^{\mathrm{nd}} \otimes \mathbb{Q}$.

(c) $\epsilon_{Y}$ is fully faithful: Use Exercise above and Lemma in 1.14 (combined with (1.8.1) and (1.13.1)) to compute the respective Ext's.

(d) $\epsilon_{Y}$ is essentially surjective: By (c), it suffices to check that for every $\left(\mathcal{F}, \varphi_{\mathcal{F}}\right) \in D_{\varphi}^{\mathrm{pcr}}(Y)^{\text {nd }}$ the cohomology $H^{i} \mathcal{F}$ belong to $D_{\varphi}^{\mathrm{pcr}}(Y)^{\text {nd }} \otimes \mathbb{Q}$ and lie in the image of $\epsilon_{Y}$. Consider $\mathcal{F}\left(E, \mathcal{L}_{E}\right):=\operatorname{holim}_{n} \mathcal{F}\left(\left(E, \mathcal{L}_{E}\right)_{n}\right)$. This is a perfect $R$-complex equipped with a nondegenerate $\varphi$-action, so $\mathcal{F}\left(E, \mathcal{L}_{E}\right) \in$ $D_{\varphi}^{\text {perf }}(R)^{\text {nd }}$. The $\varphi$-action on $R$ satisfies conditions of Proposition in $1.13{ }^{35}$ So, by (1.13.1), $H^{i} \mathcal{F}\left(E, \mathcal{L}_{E}\right) \otimes \mathbb{Q}$, viewed as an $R$-module equipped with $\varphi$-action, can be written in a canonical way as $V \otimes_{W} R$, where $V$ is an effective $\varphi$-module. Then $\nabla_{t_{l} \partial_{t_{l}}} \in \operatorname{End}_{K_{0}} H^{i} \mathcal{F}\left(E, \mathcal{L}_{E}\right) \otimes \mathbb{Q}$ preserves $V$ (as follows, say, from part (a) of the proof of loc. cit.); set $N_{V}:=\left.\nabla_{t_{l} \partial_{t_{l}}}\right|_{V}$. Then $\left(V, \varphi_{V}, N_{V}\right)$ is an effective $(\varphi, N)$-module and $H^{i} \mathcal{F}=\epsilon_{Y}(V)$, q.e.d.

Remarks. (i) To construct $\left(L i_{\text {crys }}^{0 *}\right)^{-1}$ from (1.15.2), pick a left inverse $\pi:(Y, \mathcal{L}) \rightarrow\left(Y^{0}, \mathcal{L}^{0}\right)$ to $i^{0}$ (it is not unique if $\left.Y \neq Y^{0}\right)$. Then $L \pi_{\text {crys }}^{*}$ : $D_{\varphi}^{\mathrm{pcr}}\left(Y^{0}\right)^{\mathrm{nd}} \otimes \mathbb{Q} \rightarrow D_{\varphi}^{\mathrm{pcr}}(Y)^{\mathrm{nd}} \otimes \mathbb{Q}$ is left inverse, hence inverse, to $L i_{\text {crys }}^{0 *}$.

(ii) For $\mathcal{F} \in D_{\varphi}^{\mathrm{pcr}}(Y)^{\text {nd }}$, the complex $\epsilon_{Y}^{-1}(\mathcal{F})$ as an object of $D_{\varphi}(W) \otimes \mathbb{Q}$ is equal to $L i_{\text {crys }}^{0 *}(\mathcal{F})\left(\operatorname{Spec} W, \mathcal{L}_{W}^{0}\right)$, where $\mathcal{L}_{W}^{0}$ is the $\log$ structure on $W$ defined by the prelog one $\mathcal{L}^{0} \rightarrow k \rightarrow W$, the right arrow is the Teichmüller section. ${ }^{36}$

(iii) For a map $f:\left(Y^{\prime}, \mathcal{L}^{\prime}\right) / k^{\prime} \rightarrow(Y, \mathcal{L}) / k$ of data as above its ramification index is a number $e$ such that the map $\mathbb{Z}_{\geq 0}=\mathcal{L} / \mathcal{O}_{Y}^{\times} \stackrel{f^{*}}{\rightarrow} \mathcal{L}^{\prime} / \mathcal{O}_{Y^{\prime}}^{\times}=\mathbb{Z}_{\geq 0}$ is multiplication by $e$. One has $L f_{\text {crys }}^{*} \epsilon_{Y}\left(V^{*}, \varphi, N\right)=\epsilon_{Y^{\prime}}\left(V_{K_{0}^{\prime}}, \varphi, e N\right)$ where $V_{K_{0}^{\prime}}^{\prime}:=K_{0}^{\prime} \otimes_{K_{0}} V^{\prime}$.

1.16. Hyodo-Kato theory. Let $K$ be a $p$-adic field, i.e., a complete discretely valued field of characteristic 0 with perfect residue field $k=O_{K} / \mathfrak{m}_{K}$. Let $\mathcal{L}=\mathcal{L}_{K}$ be the canonical log structure on $S=S_{K}:=\operatorname{Spec} O_{K}$ generated

${ }^{35}$ To check that the $\varphi$-action on $I=t_{l} R$ is strongly topologically nilpotent, use the finite filtration $I \supset t_{l} I \supset t_{l}^{2} I \supset \ldots \supset t_{l}^{n} I$, where $n$ is sufficiently large.

${ }^{36} \mathrm{By}$ the theorem, it is enough to identify $L i_{\text {crys }}^{0 *}\left(\epsilon_{Y}\left(V^{\cdot}\right)\right)\left(\operatorname{Spec} W, \mathcal{L}_{W}^{0}\right):=$ $\left(\epsilon_{Y^{0}}\left(V^{\cdot}\right)\right)\left(\operatorname{Spec} W, \mathcal{L}_{W}^{0}\right)$ with $V^{\cdot}$, for $V^{\cdot}$ as in (b) of the proof. We identify (Spec $\left.W, \mathcal{L}_{W}^{0}\right)$ with the exact log pd-subscheme of $\left(E, \mathcal{L}_{E}\right)$ whose ideal is generated by $t_{l}$; then $\left(\epsilon_{Y^{0}}\left(V^{\cdot}\right)\right)\left(\operatorname{Spec} W, \mathcal{L}_{W}^{0}\right)=\left(\epsilon_{Y^{0}}\left(V^{\cdot}\right)\right)\left(E, \mathcal{L}_{E}\right) \otimes_{R} W=V_{R} \otimes W=V^{*}$ (the first equality is the crystal property), q.e.d. 
by prelog one $O_{K} \backslash\{0\} \rightarrow O_{K}$. As in 1.12 , we have $\log W_{n}(k)$-schemes $(S, \mathcal{L})_{n}$. Let $v$ be the valuation on $K$ normalized so that $v(p)=1$; thus $v: \mathcal{L}_{n} /\left(O_{K} / p^{n}\right)^{\times} \stackrel{\sim}{\rightarrow} e_{K}^{-1} \mathbb{Z}_{\geq 0}$, where $e_{K}$ is the absolute ramification index, i.e., the degree of $K$ over $\bar{K}_{0}=$ Frac $W(k)$.

Let $f:\left(Z_{1}, \mathcal{M}_{1}\right) \rightarrow\left(S_{1}, \mathcal{L}_{1}\right)$ be a log smooth map of Cartier type with $\left(Z_{1}, \mathcal{M}_{1}\right)$ fine and $Z_{1}$ is proper over $S_{1}$. By the theorem in $1.14,{ }^{37}$ $R f_{\text {crys* }}\left(\mathcal{O}_{Z_{1} / \mathbb{Z}_{p}}\right)$ is a nondegenerate perfect F-crystal on $\left(S_{1}, \mathcal{L}_{1}\right)_{\text {crys }}$. Let $f^{0}:\left(Z_{1}^{0}, \mathcal{M}_{1}^{0}\right) \rightarrow\left(S_{1}^{0}, \mathcal{L}_{1}^{0}\right)$ be the pullback of $\left(Z_{1}, \mathcal{M}_{1}\right)$ by the exact embedding $i^{0}:\left(S_{1}^{0}, \mathcal{L}_{1}^{0}\right) \hookrightarrow\left(S_{1}, \mathcal{L}_{1}\right), S_{1}^{0}=\operatorname{Spec} k$, so $L i_{\text {crys }}^{0 *} R f_{\text {crys* }}\left(\mathcal{O}_{Z_{1} / \mathbb{Z}_{p}}\right)=$ $R f_{\text {crys* }}^{0}\left(\mathcal{O}_{Z_{1}^{0} / \mathbb{Z}_{p}}\right)($ see $(1.11 .1))$.

Choose $(Y, \mathcal{L})$ in 1.15 to be either of $\log k$-schemes $\left(S_{1}, \mathcal{L}_{1}\right)$ and $\left(S_{1}^{0}, \mathcal{L}_{1}^{0}\right)$; by the theorem in loc. cit., we have equivalences $\epsilon_{S_{1}}$ and $\epsilon_{S_{1}^{0}}=L i_{\text {crys }}^{0 *} \epsilon_{S_{1}}$. We define the Hyodo-Kato complex ${ }^{38} R \Gamma_{\mathrm{HK}}\left(Z_{1}^{0}, \mathcal{M}_{1}^{0}\right) \in D_{\varphi, N}\left(K_{0}\right)$ as $\epsilon_{S_{1}}^{-1} R f_{\text {crys* }}\left(\mathcal{O}_{Z_{1} / \mathbb{Z}_{p}}\right)=\epsilon_{S_{1}^{0}}^{-1} R f_{\text {crys* }}^{0}\left(\mathcal{O}_{Z_{1}^{0} / \mathbb{Z}_{p}}\right)$ with operator $N$ replaced by $e_{K}^{-1} N$.

Remark. The normalization of $N$ was chosen to make the Hyodo-Kato complex compatible with base change: If $K^{\prime}$ is a finite extension of $K$, $S^{\prime}=\operatorname{Spec} O_{K^{\prime}}$, etc., and $\left(Z_{1}^{\prime}, \mathcal{M}_{1}^{\prime}\right) /\left(S_{1}^{\prime}, \mathcal{L}_{1}^{\prime}\right)$ is the pullback of $\left(Z_{1}, \mathcal{M}_{1}\right)$, then $R \Gamma_{\mathrm{HK}}\left(Z_{1}^{\prime 0}, \mathcal{M}_{1}^{\prime 0}\right)=R \Gamma_{\mathrm{HK}}\left(Z_{1}^{0}, \mathcal{M}_{1}^{0}\right) \otimes K_{0}^{\prime}$ by (1.11.1) and Remark (iii) in 1.15 .

Let us show that the Hyodo-Kato complex controls, up to isogeny, the relative log crystalline cohomology of base changes of $f$. Let $\theta:\left(S_{1}, \mathcal{L}_{1}\right) \rightarrow$ $\left(S_{1}, \mathcal{L}_{1}\right)$ be a map of integral quasi-coherent log schemes, $\left(E^{\sim}, \mathcal{L}_{E}\right)=\varliminf\left(E_{n}^{\sim}\right.$, $\left.\mathcal{L}_{\tilde{E}_{n}}\right)$ be a $p$-adic pd-thickening of $\left(S_{1}^{\sim}, \mathcal{L}_{1}^{\tilde{1}}\right)$ over $W(k)$. Suppose $S_{1}^{\sim}$ is affine, so we have a $p$-adic ring $A$ equipped with a pd-ideal $I$ such that Spec $A / I=$ $S_{1}^{\tilde{1}}, E_{n}^{\tilde{n}}=\operatorname{Spec} A_{n}, A_{n}:=A / p^{n}$. Let $f^{\sim}:\left(Z_{1}^{\tilde{1}}, \mathcal{M}_{1}^{\tilde{1}}\right) \rightarrow\left(S_{1}^{\tilde{1}}, \mathcal{L}_{1}^{\tilde{1}}\right)$ be the $\theta$-base change of $f$. Consider the $A$-complex $R \Gamma_{\text {crys }}\left(\left(Z_{1}^{\sim} / E^{\sim}\right)_{\text {crys }}^{\log }, \mathcal{O}_{Z_{\tilde{1}} / E^{-}}\right)=$ $\operatorname{holim}_{n} R \Gamma_{\text {crys }}\left(\left(Z_{1}^{\tilde{1}} / E_{n}^{\sim}\right)_{\text {crys }}^{\log }, \mathcal{O}_{Z_{1}^{\sim} / E_{n}^{\tilde{n}}}\right)$. Any endomorphism $\varphi=\varphi_{E^{\sim}}$ of $\left(E^{\sim}, \mathcal{L}_{\tilde{E}}\right)$ that lifts the Frobenius endomorphism of $\left(S_{1}^{\tilde{1}}, \mathcal{L}_{1}^{\tilde{1}}\right)$ and is compatible with $\varphi$ on $W(k)$, acts naturally on our $A$-complex.

The datum of $\theta$ and $\left(E^{\sim}, \mathcal{L}_{\tilde{E}}\right)$ yields the Fontaine-Hyodo-Kato $\mathbb{G}_{a}$-torsor $\operatorname{Spec} A_{\mathbb{Q}}^{\tau}$ over Spec $A_{\mathbb{Q}}, A_{\mathbb{Q}}:=A \otimes \mathbb{Q} \cdot{ }^{39}$ Namely, let $\mathcal{L}_{\theta}$ be the monoid of pairs $\left(l, l^{\sim}\right)$ where $l \in \mathcal{L}_{1}$ and $l^{\sim} \in \Gamma\left(E^{\sim}, \mathcal{L}_{\tilde{E}}\right)$ lifts the pullback of $l$ in $\mathcal{L}_{1}^{\tilde{1}}$. We usually abbreviate $\left(l, l^{\sim}\right)$ to $l^{\sim}$ and write $v\left(l^{\sim}\right):=v(l) \in \mathbb{Q}$. Our $\mathcal{L}_{\theta}^{\sim}$ is an extension of $\mathcal{L}_{1}$ by $(1+I)^{\times}$, and there is a natural embedding $k^{\times} \hookrightarrow \mathcal{L}_{\tilde{\theta}}, a \mapsto\left(l_{a}, l_{a}\right):=$ the images of the Teichmüller element $[a] \in W(k)^{\times}$in $\left(O_{K} / p\right)^{\times}, A^{\times}$. Then

\footnotetext{
${ }^{37}$ applied to $Z=Z_{1}, Y=S_{1}$, and $S=\operatorname{Spf}\left(\mathbb{Z}_{p}\right)$.

38 Remark (ii) in 1.15 implies that this definition amounts to the original one from $[\mathrm{HK}] \S 3$.

${ }^{39}$ Cf. [K2] 33.
} 
$A_{\mathbb{Q}}^{\tau}$ is an $A_{\mathbb{Q}^{-}}$-algebra equipped with a map of monoids $\log : \mathcal{L}_{\theta} / k^{\times} \rightarrow A_{\mathbb{Q}}^{\tau}$ that coincides on $(1+I)^{\times}$with the composition $(1+I)^{\times} \rightarrow I \rightarrow A_{\mathbb{Q}} \rightarrow A_{\mathbb{Q}}^{\tau}$, where the left arrow is the logarithm defined by the pd structure, and such that this datum is universal. Let $N$ be the $A_{\mathbb{Q}}$-derivation of $A_{\mathbb{Q}}^{\tau}$ such that $N\left(\log \left(l^{\sim}\right)\right)=-v\left(l^{\sim}\right)$. Since $N$ is locally nilpotent, Spec $A_{\mathbb{Q}}^{\tau}$ carries a $\mathbb{G}_{a}$-action that integrates $N$ (i.e., $N$ acts as the standard generator of Lie $\mathbb{G}_{a}$ ).

Lemma. (i) Spec $A_{\mathbb{Q}}^{\tau}$ is a $\mathbb{G}_{a}$-torsor over Spec $A_{\mathbb{Q}}$.

(ii) Any Frobenius lifting $\varphi=\varphi_{E^{\sim}}$ as above acts naturally on $A_{\mathbb{Q}}^{\tau}$, and $N \varphi=$ $p \varphi N$.

Proof. Since $v:\left(\mathcal{L}_{1} / k^{\times}\right)^{\text {gr }} \otimes \mathbb{Q} \stackrel{\sim}{\rightarrow} \mathbb{Q}$, for any $l^{\sim} \in \mathcal{L}_{\tilde{\theta}}$ with $v\left(l^{\sim}\right) \neq 0$ the element $\log \left(l^{\sim}\right)$ freely generates $A_{\mathbb{Q}}^{\tau}$ over $A_{\mathbb{Q}}$. This implies (i). Then $\varphi$ acts on $A_{\mathbb{Q}}^{\tau}$ by transport of structure, and (ii) follows since $v\left(\varphi\left(l^{\sim}\right)\right)=p v\left(l^{\sim}\right)$.

The Hyodo-Kato complex carries a natural $\mathbb{G}_{a}$-action that integrates the nilpotent endomorphism $N$. Let $R \Gamma_{\mathrm{HK}}\left(Z_{1}^{0}, \mathcal{M}_{1}^{0}\right)_{A_{\mathbb{Q}}}$ be its twist by the Fontaine-Hyodo-Kato torsor. Explicitly, $R \Gamma_{\mathrm{HK}}\left(Z_{1}^{0}, \mathcal{M}_{1}^{0}\right)_{A_{\mathbb{Q}}}^{\tau}=\left(R \Gamma_{\mathrm{HK}}\left(Z_{1}^{0}\right.\right.$, $\left.\left.\mathcal{M}_{1}^{0}\right) \otimes_{K_{0}} A_{\mathbb{Q}}^{\tau}\right)^{N=0}$, where $N$ acts as $N \otimes \mathrm{id}+\mathrm{id} \otimes N$. Another description: Any $l^{\sim} \in \mathcal{L}_{\tilde{\theta}}^{\tilde{\theta}} / k^{\times}$such that $v\left(l^{\sim}\right) \neq 0$ yields a trivialization $s_{l^{\sim}}$ of the torsor defined by the equation $\log \left(l^{\sim}\right)\left(s_{l^{\sim}}\right)=0$; one has $s_{l^{\prime}}=s_{l^{\sim}}+a$, where $a=a\left(l^{\sim}, l^{\prime \sim}\right):=v\left(l^{\sim}\right)^{-1} \log \left(l^{\sim}\right)-v\left(l^{\prime \sim}\right)^{-1} \log \left(l^{\prime \sim}\right) \in A_{\mathbb{Q}}$. Thus every $l^{\sim}$ as above yields an identification $\alpha_{l^{\sim}}: R \Gamma_{\mathrm{HK}}\left(Z_{1}^{0}, \mathcal{M}_{1}^{0}\right) \otimes_{K_{0}} A_{\mathbb{Q}} \stackrel{\stackrel{\sim}{\rightarrow}}{\rightarrow} R \Gamma_{\mathrm{HK}}\left(Z_{1}^{0}, \mathcal{M}_{1}^{0}\right)_{A_{\mathbb{Q}}}$, and $\alpha_{l^{\prime}}=\alpha_{l^{\sim}} \exp \left(a\left(l^{\prime \sim}, l^{\sim}\right) N\right)$.

Theorem. (i) The A-complex $R \Gamma_{\text {crys }}\left(\left(Z_{1}^{\tilde{1}} / E^{\sim}\right)_{\text {crys }}^{\log }, \mathcal{O}_{Z_{1}^{\sim}} / E^{\sim}\right)$ is perfect, and one has $R \Gamma_{\text {crys }}\left(\left(Z_{1}^{\tilde{1}} / E_{n}^{\sim}\right)_{\text {crys }}^{\log }, \mathcal{O}_{Z_{1}^{\tilde{1}} / E_{n}^{\sim}}\right)=R \Gamma_{\text {crys }}\left(\left(Z_{1}^{\tilde{1}} / E^{\sim}\right)_{\text {crys }}^{\log }, \mathcal{O}_{Z_{1}^{\tilde{1}} / E^{\sim}}\right) \otimes{ }^{L} \mathbb{Z} / p^{n}$. (ii) There is a canonical Hyodo-Kato quasi-isomorphism of $A_{\mathbb{Q}}$-complexes

$$
\iota: R \Gamma_{H K}\left(Z_{1}^{0}, \mathcal{M}_{1}^{0}\right)_{A_{\mathbb{Q}}}^{\tau} \stackrel{\sim}{\rightarrow} R \Gamma_{\text {crys }}\left(\left(Z_{1}^{\sim} / E^{\sim}\right)_{\text {crys }}^{\log }, \mathcal{O}_{Z_{1}^{\sim} / E^{\sim}}\right) \otimes \mathbb{Q} .
$$

If we have a Frobenius lifting $\varphi_{E^{\sim}}$, then $\iota$ commutes with its action.

Proof. (i) By the theorem in 1.11 and Exercise in loc. cit., $R f_{\text {crys } *}^{\sim}\left(\mathcal{O}_{Z_{1}^{\sim} / \mathbb{Z}_{p}}\right)$ is a perfect $\mathcal{O}_{S_{1} / \mathbb{Z}_{p}}$-crystal. Its value on $\left(S_{1}^{\sim}, E_{n}^{\sim}\right)$ equals $R \Gamma_{\text {crys }}\left(\left(Z_{1}^{\tilde{1}} / E_{n}^{\sim}\right)_{\text {crys }}^{\log }\right.$, $\left.\mathcal{O}_{Z_{1} / E_{n}}\right)$. So the latter complex is $A_{n}$-perfect and $R \Gamma_{\text {crys }}\left(\left(Z_{1}^{\tilde{1}} / E_{n+1}^{\tilde{n}}\right)_{\text {crys }}^{\log }\right.$, $\left.\mathcal{O}_{Z_{1}^{\tilde{1}} / E_{n+1}^{\tilde{n}+1}}\right) \otimes_{A_{n+1}}^{L} A_{n}=R \Gamma_{\text {crys }}\left(\left(Z_{1}^{\tilde{1}} / E_{n}^{\sim}\right)_{\text {crys }}^{\log }, \mathcal{O}_{Z_{1}^{\tilde{1}} / E_{n}^{\sim}}\right)$. This implies (i) since $R \Gamma_{\text {crys }}\left(\left(Z_{1}^{\tilde{1}} / E\right)_{\text {crys }}^{\log }, \mathcal{O}_{Z_{1}^{\sim} / E^{\sim}}\right)=\operatorname{holim}_{n} R \Gamma_{\text {crys }}\left(\left(Z_{1}^{\tilde{1}} / E_{n}^{\sim}\right)_{\text {crys }}^{\log }, \mathcal{O}_{Z_{1}^{\sim} / E_{n}^{\sim}}\right)$.

(ii) To define $\iota$, it suffices to have isomorphisms $\iota_{l^{\sim}}=\iota \alpha_{l^{\sim}}: R \Gamma_{\mathrm{HK}}\left(Z_{1}^{0}\right.$, $\left.\mathcal{M}_{1}^{0}\right) \otimes_{K_{0}} A_{\mathbb{Q}} \stackrel{\sim}{\rightarrow} R \Gamma_{\text {crys }}\left(\left(Z_{1}^{\tilde{1}} / E^{\sim}\right)_{\text {crys }}^{\log }, \mathcal{O}_{Z_{1}^{\sim} / E^{\sim}}\right) \otimes \mathbb{Q}$ such that $\iota_{l^{\prime}}=\iota^{\sim} \exp \left(a\left(l^{\prime \sim}\right.\right.$, $\left.l^{\sim}\right) N$ ) for $l^{\sim}$ in a given nonempty subset of $\mathcal{L}_{\theta}^{\sim} / k^{\times}$. We take the subset of $l^{\sim}=\left(l, l^{\sim}\right)$ with $v(l)=e_{K}^{-1}$. 
We use the notation of 1.15. Picking an element $l \in \mathcal{L}_{1} / k^{\times}$such that $v(l)=e_{K}^{-1}$ amounts to a choice of $\pi_{l}:\left(S_{1}, \mathcal{L}_{1}\right) \rightarrow\left(S_{1}^{0}, \mathcal{L}_{1}^{0}\right)$ left inverse to $i^{0}:\left(S_{1}^{0}, \mathcal{L}_{1}^{0}\right) \hookrightarrow\left(S_{1}, \mathcal{L}_{1}\right)$ (we assign to $\pi_{l}$ the $\pi_{l}^{*}$-image of a generator $l^{0}$ of $\left.\mathcal{L}_{1}^{0}\right)$. Picking $\left(l, l^{\sim}\right) \in \mathcal{L}_{\theta} / k^{\times}$amounts to a choice of a morphism of pd-thickenings $\theta_{l^{\sim}}: E^{\sim} \rightarrow E$ that extends $\pi_{l} \theta$ (we assign to $\theta_{l^{\sim}}$ the $\theta_{l^{*}}^{*}$-image of the generator $t_{l^{0}}$ of $\mathcal{L}_{E} / k^{\times}$, see part (b) of the proof of the theorem in 1.15). By Remark (i) in $1.15, L \pi_{\text {lcrys }}^{*} \epsilon_{S_{1}^{0}} R \Gamma_{\mathrm{HK}}\left(Z_{1}^{0}, \mathcal{M}_{1}^{0}\right) \stackrel{\sim}{\rightarrow} R f_{\text {crys* }}\left(\mathcal{O}_{Z_{1} / \mathbb{Z}_{p}}\right) \otimes \mathbb{Q}$, so, by the theorem in 1.11, $L\left(\pi_{l} \theta\right)_{\text {crys }}^{*} \epsilon_{S_{1}^{0}} R \Gamma_{\mathrm{HK}}\left(Z_{1}^{0}, \mathcal{M}_{1}^{0}\right) \stackrel{\sim}{\rightarrow} R f_{\text {crys } *}^{\sim}\left(\mathcal{O}_{Z_{1}} / \mathbb{Z}_{p}\right) \otimes \mathbb{Q}$. Evaluating on $\left(S_{1}^{\tilde{1}}, E_{n}^{\tilde{n}}\right)$ and using Exercise in 1.11, we get $L \theta_{l^{*}}^{*}\left(\epsilon_{S_{1}^{0}}\left(R \Gamma_{\mathrm{HK}}\left(Z_{1}^{0}, \mathcal{M}_{1}^{0}\right)\right)\left(S_{1}^{0}, E\right)\right) \stackrel{\sim}{\rightarrow}$ $R \Gamma_{\text {crys }}\left(\left(Z_{1}^{\tilde{1}} / E^{\sim}\right)_{\text {crys }}^{\log }, \mathcal{O}_{Z_{1} / E^{\sim}}\right) \otimes \mathbb{Q}$. By part (b) of the proof of the theorem in 1.15 , one has $\epsilon_{S_{1}^{0}}\left(R \Gamma_{\mathrm{HK}}\left(Z_{1}^{0}, \mathcal{M}_{1}^{0}\right)\right)\left(S_{1}^{0}, E\right)=R \Gamma_{\mathrm{HK}}\left(Z_{1}^{0}, \mathcal{M}_{1}^{0}\right) \otimes_{W(k)} R$, so the previous isomorphism can be rewritten as identification $R \Gamma_{\mathrm{HK}}\left(Z_{1}^{0}, \mathcal{M}_{1}^{0}\right) \otimes_{K_{0}}$ $A_{\mathbb{Q}} \stackrel{\sim}{\rightarrow} R \Gamma_{\text {crys }}\left(\left(Z_{1}^{\tilde{1}} / E^{\sim}\right)_{\text {crys }}^{\log }, \mathcal{O}_{Z_{1} / E^{\sim}}\right) \otimes \mathbb{Q}$, which is the promised $\iota_{l}$.

The compatibility between $\iota^{2}$ s for different $l$ follows from the description of the connection on $\epsilon_{S_{1}^{0}}\left(R \Gamma_{\mathrm{HK}}\left(Z_{1}^{0}, \mathcal{M}_{1}^{0}\right)\right)\left(S_{1}^{0}, E\right)$ in terms of $N$, see the proof of the theorem in 1.15. Precisely, it suffices to compare the compositions of $\iota^{\sim}$ and $\iota_{l^{\prime}}$ with a power of the Frobenius action on $R \Gamma_{\mathrm{HK}}\left(Z_{1}^{0}, \mathcal{M}_{1}^{0}\right)$. Choose $n$ such that $l^{p^{n}}=l^{\prime p^{n}}$. Then $\operatorname{Fr}^{n} \pi_{l} \theta=F r^{n} \pi_{l^{\prime}} \theta$, hence we can compare $\varphi^{n} \theta_{l^{r}}$ with $\varphi^{n} \theta_{l^{\prime}}$ integrating the connection, q.e.d.

Example. Let $(Z, \mathcal{M})$ be a fine $\log$ scheme $\log$ smooth over $(S, \mathcal{L})$ such that $Z$ is proper over $S$ and $(Z, \mathcal{M})_{1}=\left(Z_{1}, \mathcal{M}_{1}\right)$ is of Cartier type over $(S, \mathcal{L})_{1}$. Consider $\theta=\operatorname{id}_{\left(S_{1}, \mathcal{L}_{1}\right)}$ and $\left(E, \mathcal{L}_{E}\right)=(S, \mathcal{L})$, so $A=O_{K}$. Then $R \Gamma\left(\left(Z_{1} / S_{n}\right)_{\text {crys }}^{\log }, \mathcal{O}_{Z_{1} / S_{n}}\right)=R \Gamma\left(Z_{n}, \Omega_{(Z, \mathcal{M})_{n} /(S, \mathcal{L})_{n}}\right)$ by $(1.8 .3)$ and the invariance property of crystalline topology. Thus $R \Gamma\left(\left(Z_{1} / S\right)_{\text {crys }}^{\log }, \mathcal{O}_{Z_{1} / S}\right)=$ $R \Gamma\left(Z, \Omega_{(Z, \mathcal{M}) /(S, \mathcal{L})}\right)$, and (1.16.1) becomes the classical Hyodo-Kato quasiisomorphism [HK] 5.1 (here $Z_{K}:=Z \otimes_{O_{K}} K$ )

$$
\iota_{\mathrm{dR}}: R \Gamma_{\mathrm{HK}}\left(Z_{1}^{0}, \mathcal{M}_{1}^{0}\right)_{K}^{\tau} \stackrel{\sim}{\rightarrow} R \Gamma\left(Z_{K}, \Omega_{\left(Z_{K}, \mathcal{M}_{K}\right) / K}\right) .
$$

1.17. Absolute crystalline cohomology of $O_{\bar{K}} / p . \quad$ Let $\left(Y, \mathcal{N}_{Y}\right)$ be an integral quasi-coherent $\log$ scheme over $\mathbb{Z} / p$ such that the monoid $\mathcal{N}_{Y} / \mathcal{O}_{Y}^{\times}$is uniquely $p$-divisible. The next lemma was pointed to us by the referee:

Lemma. One has $Y_{\text {crys }}=(Y, \mathcal{N})_{\text {crys }}$, i.e., every pd-thickening $P$ of $Y$ over $\mathbb{Z} / p^{n}$ carries a unique log structure $\mathcal{N}_{P}$.

Proof. Let us construct $\mathcal{N}_{P}$; its uniqueness follows from Exercise below. Let $\mathcal{N}_{Y}^{(n)}$ be a copy of $\mathcal{N}_{Y}$, and $\alpha^{(n)}: \mathcal{N}_{Y}^{(n)} \rightarrow \mathcal{O}_{P}$ be the map $a \mapsto \tilde{\alpha}(a)^{p^{n}}$, where $\tilde{\alpha}(a)$ is any lifting of $\alpha(a) \in \mathcal{O}_{Y}$ to $\mathcal{O}_{P}$. This map is well defined 
(indeed, for $b \in \mathcal{O}_{Y}$ and $c \in \mathcal{J}_{P}$ one has $(b+c)^{p^{n}}=b^{p^{n}}$ since $\left.c^{p} \in p \mathcal{J}_{P}\right)$. It is a prelog structure on $P$; our $\mathcal{N}_{P}$ is the corresponding log structure. The map $\mathcal{N}_{Y}^{(n)} \rightarrow \mathcal{N}_{Y}, a \mapsto a^{p^{n}}$, lifts the embedding $Y \hookrightarrow P$ to an embedding of $\log$ schemes $\left(Y, \mathcal{N}_{Y}\right) \hookrightarrow\left(P, \mathcal{N}_{P}\right)$, which is exact due to the condition of the lemma.

Exercise. Show that $\mathcal{N}_{P}$ satisfies the next universal property: For any $(Z, T$, $\left.\mathcal{M}_{T}\right) \in \mathcal{T}_{\mathbb{Z} / p^{n}}$ (see 1.3$)$ every map of $\log$ schemes $\left(Z, \mathcal{M}_{Z}\right) \rightarrow\left(Y, \mathcal{N}_{Y}\right)$ and a map of pd-thickenings $T \rightarrow P$ that restrict to the same map $Z \rightarrow Y$ lift in a unique manner to a morphism log pd-thickenings $\left(Z, T, \mathcal{M}_{T}\right) \rightarrow\left(Y, P, \mathcal{N}_{P}\right)$.

As in 1.16 , set $K_{0}=$ Frac $W$, where $W=W(k), k$ is a perfect field. Let $\bar{K}$ be an algebraic closure of $K_{0}, O_{\bar{K}}$ be its ring of integers, $\bar{k}=O_{\bar{K}} / \mathfrak{m}_{\bar{K}}$ the residue field. Let $\overline{\mathcal{L}}$ be the canonical $\log$ structure on $\bar{S}:=\operatorname{Spec} O_{\bar{K}}$ generated by the prelog structure $O_{\bar{K}} \backslash\{0\} \rightarrow O_{\bar{K}}$. Let $v$ be the normalized valuation on $\bar{K}, v(p)=1$, so we have $v: \overline{\mathcal{L}} / O_{\bar{K}}^{\times}=\overline{\mathcal{L}}_{1} /\left(O_{\bar{K}} / p\right)^{\times} \stackrel{\sim}{\rightarrow} \mathbb{Q} \geq 0$.

Consider Fontaine's ring $\mathrm{A}_{\text {crys }}$ from [F1] 2.2, 2.3. This is a p-adically complete ring such that $\mathrm{A}_{\text {crys } n}=\mathrm{A}_{\text {crys }} / p^{n}$ is a universal pd-thickening of $O_{\bar{K}} / p$ over $W_{n}$. Let $J_{\operatorname{crys} n}$ be the pd-ideals, $\mathrm{A}_{\operatorname{crys} n} / J_{\operatorname{crys} n}=O_{\bar{K}} / p$. Set $E_{\text {crys } n}:=\operatorname{Spec} A_{\operatorname{crys} n}$.

The $\log$ structure $\overline{\mathcal{L}}_{1}$ on $\operatorname{Spec}\left(O_{\bar{K}} / p\right)=\bar{S}_{1}$ satisfies the condition of the lemma. So it extends in a unique manner to an integral log structure $\mathcal{L}_{\text {crys } n}$ on $E_{\text {crys } n}$. By Exercise, the pd-thickening $(\bar{S}, \overline{\mathcal{L}})_{1} \hookrightarrow\left(E_{\text {crys } n}, \mathcal{L}_{\text {crys } n}\right)$ is universal, i.e., for $(Z, T) \in \mathcal{T}_{W_{n}}$ every map $h:(Z, \mathcal{M}) \rightarrow(\bar{S}, \overline{\mathcal{L}})_{1}$ of $\log$ $k$-schemes extends in a unique way to a $\mathcal{T}_{W_{n}}$-map $h_{T}:(Z, T) \rightarrow\left(S_{1}, E_{\text {crys } n}\right)$.

The Frobenius map $\varphi$ lifts to $\left(E_{\operatorname{crys} n}, \mathcal{L}_{\operatorname{crys} n}\right)$ by universality.

The log structures $\mathcal{L}_{\text {crys } n}$ are mutually compatible. Thus we have the log structure $\mathcal{L}_{\text {crys }}=\lim _{\mathcal{L}_{\text {crys } n}}$ on $E_{\text {crys }}=\operatorname{Spec}_{\text {crys }}$ such that $\left.\mathcal{L}_{\text {crys }}\right|_{E_{\text {crys } n}}=$ $\mathcal{L}_{\text {crys } n}$. Explicitly, the identifications $\left.\mathcal{L}_{\text {crys } n}\right|_{E_{\text {crys } m}} \stackrel{\sim}{\rightarrow} \mathcal{L}_{\text {crys } m}$ for $n \geq m$ come from the maps $\overline{\mathcal{L}}_{1}^{(n)} \rightarrow \overline{\mathcal{L}}_{1}^{(m)}, l \mapsto \varphi^{n-m}(l)$, and the log structure $\mathcal{L}_{\text {crys }}$ comes

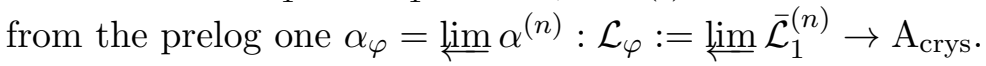

Exercise. One has $\mathcal{L}_{\varphi} \stackrel{\sim}{\rightarrow}\left\{\lambda \in \mathcal{L}_{\text {crys }}: \varphi(\lambda)=\lambda^{p}\right\} .{ }^{40}$

Our $\left(\bar{S}_{1}, E_{\operatorname{crys} n}\right)$ is a final object of the absolute crystalline site $\left(\bar{S}_{1}, \overline{\mathcal{L}}_{1}\right)_{\operatorname{crys}(n)}($ see 1.12$)$ by Lemma, so for any sheaf $\mathcal{F}$ its global sections are equal to $\mathcal{F}\left(S_{1}, E_{\operatorname{crys} n}\right)$. The étale topology of $E_{\operatorname{crys} n}$ is trivial, so the higher cohomology vanish and

$$
R \Gamma\left(\left(\bar{S}_{1}, \overline{\mathcal{L}}_{1}\right)_{\operatorname{crys}(n)}, \mathcal{F}\right)=\mathcal{F}\left(\bar{S}_{1}, E_{\text {crys } n}\right) .
$$

${ }^{40}$ Hint: $\operatorname{dim}_{\mathbb{Q}_{p}}\left\{b \in \mathrm{B}_{\text {crys }}^{+}: \varphi(b)=p b, b \in F^{1}\right\}=1$. 
Thus for a sheaf $\mathcal{F}$ on $\left(\bar{S}_{1}, \overline{\mathcal{L}}_{1}\right)_{\text {crys }}$ one has

$$
R \Gamma\left(\left(\bar{S}_{1}, \overline{\mathcal{L}}_{1}\right)_{\text {crys }}, \mathcal{F}\right)=\mathcal{F}\left(\bar{S}_{1}, E_{\text {crys }}\right):=\operatorname{holim}_{n} \mathcal{F}\left(\bar{S}_{1}, E_{\text {crys } n}\right) .
$$

In particular,

$$
R \Gamma_{\text {crys }}(\bar{S}, \overline{\mathcal{L}})_{(n)}=\mathrm{A}_{\text {crys } n}, \quad R \Gamma_{\text {crys }}(\bar{S}, \overline{\mathcal{L}})=\mathrm{A}_{\text {crys }}
$$

1.18. Absolute crystalline cohomology of log schemes over $O_{\bar{K}} / p$. Let $\bar{f}$ : $\left(Z_{1}^{-}, \mathcal{M}_{1}^{-}\right) \rightarrow\left(\bar{S}_{1}, \overline{\mathcal{L}}_{1}\right)$ be a map of log schemes, its source is integral quasicoherent. Then for any sheaf $\mathcal{F}$ on $\left(Z_{1}^{-}, \mathcal{M}_{1}^{-}\right)_{\text {crys }}$ (see 1.12) one has a natural identification

$$
R \Gamma\left(\left(Z_{1}^{-}, \mathcal{M}_{1}^{-}\right)_{\text {crys }}, \mathcal{F}\right) \stackrel{\sim}{\rightarrow} R \Gamma\left(\left(\left(Z_{1}^{-}, \mathcal{M}_{1}^{-}\right) /\left(\bar{S}_{1}, E_{\text {crys }}\right)\right)_{\text {crys }}, \mathcal{F}\right) .
$$

Namely, consider $\bar{f}_{\text {crys }}:\left(Z_{1}^{-}, \mathcal{M}_{1}^{-}\right)_{\text {crys }} \rightarrow\left(\bar{S}_{1}, \overline{\mathcal{L}}_{1}\right)_{\text {crys }}$; then the 1.h.s. of $(1.18 .1)$ is $R \Gamma\left(\left(\bar{S}_{1}, \overline{\mathcal{L}}_{1}\right)_{\text {crys }}, R \bar{f}_{\text {crys } *} \mathcal{F}\right)$, the same is true for the r.h.s. by (1.17.2).

Let $K \subset \bar{K}$ be a finite extension of $K_{0}$, and $\theta=\theta_{K}:(\bar{S}, \overline{\mathcal{L}}) \rightarrow(S, \mathcal{L})=$ $\left(S_{K}, \mathcal{L}_{K}\right)$ be the map defined by the embedding $K \hookrightarrow \bar{K}$. Suppose our $\bar{f}$ is the base change of $f:\left(Z_{1}, \mathcal{M}_{1}\right) \rightarrow(S, \mathcal{L})_{1}$ by $\theta_{1}$, i.e., we have $\theta_{Z 1}$ : $\left(Z_{1}^{-}, \mathcal{M}_{1}^{-}\right) \rightarrow\left(Z_{1}, \mathcal{M}_{1}\right)$ such that the square $\left(\bar{f}, f, \theta_{1}, \theta_{Z 1}\right)$ is Cartesian, and $f$ satisfies the properties from 1.16. Applying the theorem in 1.16 to $S_{1}=\bar{S}_{1}$, $E=E_{\text {crys }}$ and using (1.18.1) for $\mathcal{F}=\mathcal{O}_{Z_{1}^{-} / \mathbb{Z}_{p}}$, we get (recall that $\mathrm{B}_{\text {crys }}^{+}:=$ $\mathrm{A}_{\text {crys }} \otimes \mathbb{Q}$; see 1.12 for the rest of the notation):

Theorem. (i) $R \Gamma_{\text {crys }}\left(Z_{1}^{-}, \mathcal{M}_{1}^{-}\right)$is a perfect $A_{\text {crys }}$-complex, and $R \Gamma_{\text {crys }}\left(Z_{1}^{-}\right.$, $\left.\mathcal{M}_{1}^{-}\right)_{(n)}=R \Gamma_{\text {crys }}\left(Z_{1}^{-}, \mathcal{M}_{1}^{-}\right) \otimes^{L} \mathbb{Z} / p^{n}=R \Gamma_{\text {crys }}\left(Z_{1}^{-}, \mathcal{M}_{1}^{-}\right) \otimes_{A_{\text {crys }}} A_{\text {crys } n}$.

(ii) There is a canonical quasi-isomorphism of $B_{\text {crys }}^{+}$-complexes

$$
\iota_{\text {crys }}: R \Gamma_{H K}\left(Z_{1}^{0}, \mathcal{M}_{1}^{0}\right)_{B_{\text {crys }}^{+}}^{\tau} \stackrel{\sim}{\rightarrow} R \Gamma_{\text {crys }}\left(Z_{1}^{-}, \mathcal{M}_{1}^{-}\right) \otimes \mathbb{Q}
$$

compatible with the action of $\varphi$.

Remarks. (i) The ring $\mathrm{A}_{\text {crys } \mathbb{Q}}^{\tau}$ from 1.16 equals Fontaine's ring $\mathrm{B}_{\mathrm{st}}^{+}$. Indeed, by [F1] 3.1, $\mathrm{B}_{\text {st }}^{+}$is a $\mathrm{B}_{\text {crys }}^{+}$-algebra equipped with a monoid map log $: \mathcal{L}_{\text {crys }} / \bar{k}^{\times} \rightarrow$ $\mathrm{B}_{\mathrm{st}}^{+}$that equals the logarithm defined by the pd structure on $\left(1+J_{\text {crys }}\right)^{\times}$, and such that this datum is universal. Since the evident map $\mathcal{L}_{\theta} \rightarrow \mathcal{L}_{\text {crys }}$ yields an isomorphism $\left(\mathcal{L}_{\hat{\theta}} / k^{\times}\right)^{\mathrm{gr}} \otimes \mathbb{Q} \stackrel{\sim}{\rightarrow}\left(\mathcal{L}_{\text {crys }} / \bar{k}^{\times}\right)^{\mathrm{gr}} \otimes \mathbb{Q}$, the assertion follows. The derivation $N$ and the Frobenius action $\varphi$ from 1.16 coincide with those from [F1] 3.2. 
(ii) Suppose our $\bar{f}$ is such that the datum of $\left(K,\left(Z_{1}, \mathcal{M}_{1}\right), \theta_{Z 1}\right)$ as above exists, but we don't want to specify one. All such data form a category in an evident manner; since $\bar{S}_{1}$ is faithfully flat over $S_{1}$, it is an ordered set, which we denote by $\Xi_{1}$. In fact, $\Xi_{1}$ is directed. For a morphism $\left(K^{\prime},\left(Z_{1}^{\prime}, \mathcal{M}_{1}^{\prime}\right), \theta_{Z 1}^{\prime}\right) \rightarrow\left(K,\left(Z_{1}, \mathcal{M}_{1}\right), \theta_{Z 1}\right)$ in $\Xi_{1}$, one has a canonical identification $R \Gamma_{\mathrm{HK}}\left(Z_{1}^{0}, \mathcal{M}_{1}^{0}\right) \otimes_{K_{0}} K_{0}^{\prime} \stackrel{\sim}{\rightarrow} R \Gamma_{\mathrm{HK}}\left(Z_{1}^{\prime 0}, \mathcal{M}_{1}^{\prime 0}\right)$ (see Remark in 1.16), and isomorphisms (1.18.2) are compatible with it. Set $R \Gamma_{\mathrm{HK}}\left(Z_{1}^{-}, \mathcal{M}_{1}^{-}\right):=$ $\varliminf_{\Xi_{1}} R \Gamma_{\mathrm{HK}}\left(Z_{1}^{0}, \mathcal{M}_{1}^{0}\right)$; this is a complex of $(\varphi, N)$-modules over $K_{0}^{\mathrm{nr} 41}$ functorial with respect to morphisms of $X^{\prime}$ 's, and (1.8.3) provides a canonical isomorphism compatible with the action of $\varphi$

$$
\iota_{\text {crys }}: R \Gamma_{\mathrm{HK}}\left(Z_{1}^{-}, \mathcal{M}_{1}^{-}\right)_{\mathrm{B}_{\text {crys }}^{+}}^{+} \stackrel{\sim}{\rightarrow} R \Gamma_{\text {crys }}\left(Z_{1}^{-}, \mathcal{M}_{1}^{-}\right) \otimes \mathbb{Q} .
$$

Since Spec B $\mathrm{B}_{\text {st }}^{+}$is a $\mathbb{G}_{a}$-torsor over Spec B $\mathrm{B}_{\text {crys }}^{+}$(see Lemma in 1.16), (1.18.3) amounts to a quasi-isomorphism of $\mathrm{B}_{\mathrm{st}}^{+}$-complexes compatible with the action of $N$ and $\varphi$

$$
\iota_{\text {crys }}: R \Gamma_{\mathrm{HK}}\left(Z_{1}^{-}, \mathcal{M}_{1}^{-}\right)_{\mathrm{B}_{\mathrm{st}}^{+}} \stackrel{\sim}{\rightarrow} R \Gamma_{\text {crys }}\left(Z_{1}^{-}, \mathcal{M}_{1}^{-}\right) \otimes{ }_{\mathrm{A}_{\text {crys }}}^{L} \mathrm{~B}_{\mathrm{st}}^{+} .
$$

Here $R \Gamma_{\mathrm{HK}}\left(Z_{1}^{-}, \mathcal{M}_{1}^{-}\right)_{\mathrm{B}_{\mathrm{st}}^{+}}:=R \Gamma_{\mathrm{HK}}\left(Z_{1}^{-}, \mathcal{M}_{1}^{-}\right) \otimes_{K_{0}^{\mathrm{nr}}} \mathrm{B}_{\mathrm{st}}^{+}$.

(iii) Suppose $\left(Z_{1}^{-}, \mathcal{M}_{1}^{-}\right) /(\bar{S}, \overline{\mathcal{L}})_{1}$ is reduction mod $p$ of a log scheme $\left(Z^{-}, \mathcal{M}^{-}\right) /(\bar{S}, \overline{\mathcal{L}})$. Assume that there exists a datum $\left(K,(Z, \mathcal{M}), \theta_{Z}\right)$, where $K \subset \bar{K}$ is a finite extension of $K_{0},(Z, \mathcal{M}) /(S, \mathcal{L})$ is a log scheme that satisfies conditions of Example in 1.16, and $\theta_{Z}:\left(Z^{-}, \mathcal{M}^{-}\right) \rightarrow(Z, \mathcal{M})$ is an identification of $\left(Z^{-}, \mathcal{M}^{-}\right)$with the $\theta$-pullback of $(Z, \mathcal{M})$. Again, such data form a directed set $\Xi$, and the reduction $\bmod p \operatorname{map} \Xi \rightarrow \Xi_{1}$ is cofinal. Isomorphisms (1.16.2) are compatible with morphisms in $\Xi$, and their $\Xi$-colimit is a natural isomorphism (here $Z_{\bar{K}}^{\bar{K}}:=Z^{-} \otimes_{O_{\bar{K}}} \bar{K}$ )

$$
\iota_{\mathrm{dR}}: R \Gamma_{\mathrm{HK}}\left(Z_{1}^{-}, \mathcal{M}_{1}^{-}\right)_{\bar{K}}^{\tau} \stackrel{\sim}{\rightarrow} R \Gamma\left(Z_{\overline{\bar{K}}}^{\bar{\rightarrow}}, \Omega_{\left(Z_{\bar{K}}, \mathcal{M}_{\bar{K}}\right) / \bar{K}}\right) .
$$

1.19. Log de Rham complex in characteristic $0 . \quad$ Let $(Y, \mathcal{N})$ be an integral fine $\log$ scheme $\log$ smooth over a field $F$ of characteristic 0 , and $Y^{0}$ be the open subset where the $\log$ structure is trivial. The embedding $j: Y^{0} \hookrightarrow Y$ is affine. The next result is due to Ogus [Og2] 1.3; the key idea of the proof is borrowed from [D].

Theorem. If the sheaf of groups $\mathcal{N}^{g r} / \mathcal{O}_{Y}^{\times}$has trivial torsion (e.g. if $\mathcal{N}$ is saturated), then the natural map $r_{Y}: \Omega_{(Y, \mathcal{N}) / F} \rightarrow j_{*} \Omega_{Y^{0} / F}$ is a quasiisomorphism.

\footnotetext{
${ }^{41}$ The maximal unramified extension of $K_{0}$ in $\bar{K}$.
} 
Proof. (i) We want to prove that $\mathcal{C}$ one $\left(r_{Y}\right)$ is acyclic. This is a complex of quasi-coherent $\mathcal{O}_{Y}$-modules whose differentials are differential operators. Let $C_{Y}$ be corresponding complex of induced $\mathcal{D}$-modules on $Y$, see [S]; here $\mathcal{D}$-modules on a singular variety are understood in the usual way (using closed embeddings into a smooth variety). By loc. cit., Cone $\left(r_{Y}\right)$ is quasiisomorphic to the de Rham complex $\mathrm{dR}\left(C_{Y}\right)$, so it suffices to show that $C_{Y}$ is acyclic. We proceed by induction by $\operatorname{dim} Y$.

(ii) Our claim is étale local, so, by [K1] 3.5, we can assume that $Y=$ Spec $F[N]$ for a fine monoid $N, \mathcal{N}$ comes from the $N$-chart. Since $\mathcal{N}^{\mathrm{gr}} / \mathcal{O}_{Y}^{\times}$ has trivial torsion, we can assume that $N^{\text {gr }}$ has trivial torsion. Then $T:=$ Spec $F\left[N^{\mathrm{gr}}\right]$ is a torus which acts on $Y$, and $Y^{0}=T$ is the open orbit.

(iii) $C_{Y}$ is acyclic outside $Y^{T}$ : For a closed $y \in Y$ not fixed by $T$, let us find an étale neighborhood $U$ of $y$ such that $C_{U}$ is acyclic. Pick $n \in N \backslash\{0\}$ with $\alpha(n)(y) \neq 0$ and then a 1-parameter subgroup $G \subset T$ such that $\left.n\right|_{G}$ is nontrivial; set $Z:=\alpha(n)^{-1}(\{1\}) \subset Y$. Our $U$ is $G \times Z \rightarrow Y,(g, z) \mapsto g(z)$. Since $\left(U, \mathcal{N}_{U}\right)=G \times\left(Z,\left.\mathcal{N}\right|_{Z}\right), C_{U}$ is quasi-isomorphic to the pullback of $C_{Z}$ by the projection $U \rightarrow Z$; since $C_{Z}$ is acyclic by the induction assumption, $C_{U}$ is acyclic.

(iv) By (iii), $C_{Y}=i_{*} R i^{!}\left(C_{Y}\right)$ where $i: Y^{T} \hookrightarrow Y$. Since $Y^{T}$ is a single point if nonempty, $R i^{!}\left(C_{Y}\right)=R \Gamma_{\mathrm{dR}}\left(Y, C_{Y}\right)$, which is the cone of $\Gamma\left(r_{Y}\right)$ : $\Gamma\left(Y, \Omega_{(Y, \mathcal{N}) / F}\right) \rightarrow \Gamma\left(T, \Omega_{T / F}\right)$ since $Y$ is affine. It remains to check that $\Gamma\left(r_{Y}\right)$ is a quasi-isomorphism. The $T$-action on $Y$ yields an $N^{\text {gr }}$-grading on the complexes. Since $\Omega_{T / F}^{i}=\mathcal{O}_{T} \otimes \Lambda^{i} N^{\mathrm{gr}}$, the $n$-component $\Gamma\left(T, \Omega_{T / F}\right)_{n}$, $n \in N^{\mathrm{gr}}$, equals $F \otimes \Lambda^{\cdot} N^{\mathrm{gr}}$ with differential $\ell \mapsto n \wedge \ell$. Since $\Omega_{(Y, \mathcal{N}) / F}^{i}=\mathcal{O}_{Y} \otimes$ $\Lambda^{i} N^{\mathrm{gr}}$, the map $\Gamma\left(r_{Y}\right)$ is injective and its image is the sum of components $\Gamma\left(T, \Omega_{T / F}\right)_{n}$ for $n \in N$. We are done since $\Gamma\left(T, \Omega_{T / F}\right)_{n}$ is acyclic for $n \neq 0$, hence for $n \in N^{\mathrm{gr}} \backslash N$.

\section{The $h$-sheaf $\mathcal{A}_{\text {crys }}$ and the crystalline Poincaré lemma}

2.1. The next general format will be of use. Let $\mathcal{V} r_{F}$ be the category of algebraic varieties over a field $F$ (i.e., separated reduced $F$-schemes of finite type). Let $\mathcal{V} r_{F h}$ be the corresponding h-site (see [SV] or [B] 2.4); for $X \in \mathcal{V a r}_{F}$ let $X_{\mathrm{h}}$ be the h-site of $X$. For a complex $P$ of abelian groups we denote by $P_{\mathcal{V a r}_{F \mathrm{~h}}}$ the corresponding complex of constant h-sheaves; as in [B] (1.1.1), $P \widehat{\otimes} \mathbb{Z}_{p}:=\operatorname{holim}_{n} P \otimes L \mathbb{Z} / p^{n}, P \widehat{\otimes} \mathbb{Q}_{p}:=\left(P \widehat{\otimes} \mathbb{Z}_{p}\right) \otimes \mathbb{Q}$.

Let $\mathcal{A}$ be a complex of h-sheaves on $\mathcal{V}_{F}$. Set $A:=\mathcal{A}(\operatorname{Spec} F)$; one has an evident canonical morphism $A_{\mathcal{V}_{\text {ar }} \mathrm{Fh}} \rightarrow \mathcal{A}$. We say that $\mathcal{A}$ is p-adically constant if the map $\left(A \otimes^{L} \mathbb{Z} / p\right){\mathcal{V} a r_{F h}}=A_{\mathcal{V a r}_{F \mathrm{~h}}} \otimes^{L} \mathbb{Z} / p \rightarrow \mathcal{A} \otimes^{L} \mathbb{Z} / p$ is a quasi-isomorphism in the derived category of h-sheaves $D\left(\mathcal{V} a r_{F h}\right)$. 
Remarks. (i) For a $p$-adically constant $\mathcal{A}$ the map $\left(A \otimes^{L} \mathbb{Z} / p^{n}\right)_{\mathcal{V} r_{F \mathrm{~h}}} \rightarrow$ $\mathcal{A} \otimes^{L} \mathbb{Z} / p^{n}$ is automatically a quasi-isomorphism for every $n>0$.

(ii) $p$-adically constant complexes form a thick subcategory of $D\left(\mathcal{V}_{a r_{F h}}\right)$.

Proposition. (i) For a p-adically constant $\mathcal{A}$ as above and $X \in \mathcal{V} a r_{F}$, one has canonical quasi-isomorphisms

$$
R \Gamma\left(X_{h}, \mathcal{A}\right) \otimes \mathbb{Z} / p^{n} \stackrel{\sim}{\rightarrow} R \Gamma\left(X_{\text {ét }}, \mathbb{Z} / p^{n}\right) \otimes_{\mathbb{Z} / p^{n}}\left(A \otimes \mathbb{Z} / p^{n}\right) .
$$

(ii) If, in addition, $F$ is algebraically closed and $p$ is prime to char $F$, then one has a canonical quasi-isomorphism

$$
R \Gamma\left(X_{h}, \mathcal{A}\right) \widehat{\otimes} \mathbb{Z}_{p} \stackrel{\sim}{\rightarrow} R \Gamma_{e ́ t}\left(X, \mathbb{Z}_{p}\right) \otimes_{\mathbb{Z}_{p}}\left(A \widehat{\otimes} \mathbb{Z}_{p}\right) .
$$

If $\mathcal{A}$ is an $E_{\infty}$ algebra, then these are quasi-isomorphisms of $E_{\infty}$ algebras.

Proof. (i) (2.1.1) is the composition of canonical quasi-isomorphisms $R \Gamma\left(X_{\mathrm{h}}\right.$,

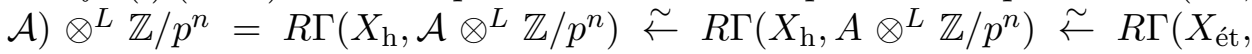
$\left.A \otimes^{L} \mathbb{Z} / p^{n}\right) \leftarrow R \Gamma\left(X_{\text {ét }}, \mathbb{Z} / p^{n}\right) \otimes_{\mathbb{Z} / p^{n}}^{L}\left(A \otimes^{L} \mathbb{Z} / p^{n}\right)$, the first $\sim$ comes from Remark (i), the second one comes since, by Deligne's cohomological descent, the étale and h-cohomology with torsion coefficients coincide (see Remark in [B] 3.4), the third one is [G] 3.3.

(ii) By the condition on $F$, the complex $R \Gamma_{\text {ét }}\left(X, \mathbb{Z}_{p}\right):=\operatorname{holim}_{n} R \Gamma\left(X_{\text {ét }}\right.$, $\left.\mathbb{Z} / p^{n}\right)$ is $\mathbb{Z}_{p}$-perfect and $R \Gamma_{\text {ét }}\left(X, \mathbb{Z}_{p}\right) \otimes_{\mathbb{Z}_{p}}^{L} \mathbb{Z} / p^{n} \stackrel{\sim}{\rightarrow} R \Gamma\left(X_{\text {ét }}, \mathbb{Z} / p^{n}\right)$. Thus (2.1.1) can be rewritten as $R \Gamma\left(X_{\mathrm{h}}, \mathcal{A}\right) \otimes^{L} \mathbb{Z} / p^{n} \stackrel{\sim}{\rightarrow} R \Gamma_{\text {ét }}\left(X, \mathbb{Z}_{p}\right) \otimes_{\mathbb{Z}_{p}}^{L}\left(A \otimes \otimes^{L} \mathbb{Z} / p^{n}\right)$. Applying $\operatorname{holim}_{n}$, we get (2.1.2).

2.2. From now on $K$ is a $p$-adic field as in 1.16. Let $(V, \bar{V})$ be an ss-pair over $\bar{K}$, see [B] $2.2(\mathrm{c})$. As in [B] 3.2, we view it as a log $W(k)$-scheme with underlying scheme $\bar{V}$. The final object $\operatorname{Spec}\left(\bar{K}, O_{\bar{K}}\right)$ of $\mathcal{V}_{\bar{K}} r_{\bar{K}}^{\text {ss }}$ is $(\bar{S}, \overline{\mathcal{L}})$ (see $1.17)$, so $(V, \bar{V})$ is a log scheme over $(\bar{S}, \overline{\mathcal{L}})$.

As in 1.12, one has the absolute log crystalline complexes $R \Gamma_{\text {crys }}(V, \bar{V})_{(n)}$ and $R \Gamma_{\text {crys }}(V, \bar{V})=\operatorname{holim}_{n} R \Gamma_{\text {crys }}(V, \bar{V})_{(n)} \cdot \operatorname{By}(1.17 .3), R \Gamma_{\text {crys }}(\operatorname{Spec}(\bar{K}$, $\left.\left.O_{\bar{K}}\right)\right)=\mathrm{A}_{\text {crys }}$.

According to the lemma in [B] 4.1, there is a finite extension $K$ of $K_{0}, K \subset \bar{K}$, and a $\log$ smooth integral map $f:(Z, \mathcal{M}) \rightarrow(S, \mathcal{L})=$ $\left(\right.$ Spec $\left.O_{K}, \mathcal{L}_{K}\right)$ with $f_{1}$ of Cartier type, together with an identification of $(V, \bar{V}) /(\bar{S}, \overline{\mathcal{L}})$ with the pullback of $(Z, \mathcal{M}) /(S, \mathcal{L})$ by $\theta:(\bar{S}, \overline{\mathcal{L}}) \rightarrow(S, \mathcal{L})$ (see 1.18). By (i) of Theorem in 1.18, one has:

Proposition. $R \Gamma_{\text {crys }}(V, \bar{V})$ is a perfect $A_{\text {crys }}$ complex and $R \Gamma_{\text {crys }}(V, \bar{V}) \otimes^{L}$ $\mathbb{Z} / p^{n} \stackrel{\sim}{\rightarrow} R \Gamma_{\text {crys }}(V, \bar{V})_{(n)}$. 
Remark. Enlarging $K$, we find that any finite diagram of $(V, \bar{V})$ 's comes from a diagram of $(Z, \mathcal{M})$ 's over common $(S, \mathcal{L})$.

2.3. Let $\mathcal{A}_{\text {crys }}$ be h-sheafification of the presheaf $(V, \bar{V}) \mapsto R \Gamma_{\text {crys }}(V, \bar{V})$ on $\mathcal{V} a r_{\bar{K}}^{\text {ss }}$ (see [B] 2.6). ${ }^{42}$ This is an h-sheaf of $\mathrm{E}_{\infty} \mathrm{A}_{\text {crys-algebras on } \mathcal{V} r_{\bar{K}}}$ equipped with the Frobenius action $\varphi$. Since h-sheafification is exact, $\mathcal{A}_{\text {crys } n}:=\mathcal{A}_{\text {crys }} \otimes^{L} \mathbb{Z} / p^{n}$ equals the h-sheafification of the presheaf $(V, \bar{V}) \mapsto$ $R \Gamma_{\text {crys }}(V, \bar{V})_{(n)}$ by Proposition in 2.2 .

Theorem (the crystalline Poincaré lemma). $\mathcal{A}_{\text {crys }}$ is p-adically constant.

Proof. Since $\left(\mathrm{A}_{\text {crys } 1}\right)_{\mathcal{V a r}_{\bar{K} \mathrm{~h}}} \stackrel{\sim}{\rightarrow} H^{0} \mathcal{A}_{\text {crys } 1}$, we need to show that $H^{>0} \mathcal{A}_{\text {crys } 1}=$ 0 . It suffices to show that every $(V, \bar{V}) \in \mathcal{V} a r_{\bar{K}}^{\mathrm{ss}}$ admits an h-covering $\left(V^{\prime}, \bar{V}^{\prime}\right) \rightarrow$ $(V, \bar{V})$ such that the map $H^{>0} R \Gamma_{\text {crys }}(V, \bar{V})_{(1)} \rightarrow H^{>0} R \Gamma_{\text {crys }}\left(V^{\prime}, \bar{V}^{\prime}\right)_{(1)}$ vanishes. By the next lemma, any composition of $\operatorname{dim} V+1$ p-negligible hcoverings (see [B] 4.3) does the job, so we are done by the theorem in [B] 4.3 .

Let $\left(V^{m+1}, \bar{V}^{m+1}\right) \stackrel{\psi_{m}}{\longrightarrow} \ldots \stackrel{\psi_{1}}{\longrightarrow}\left(V^{1}, \bar{V}^{1}\right)$ be p-negligible maps in $\mathcal{V} a r_{\bar{K}}^{\mathrm{ss}}$.

Lemma. The composition $\tau_{>0} R \Gamma_{\text {crys }}\left(V^{1}, \bar{V}^{1}\right)_{(1)} \rightarrow \tau_{>0} R \Gamma_{\text {crys }}\left(V^{m+1}, \bar{V}^{m+1}\right)_{(1)}$ vanishes if $m>\operatorname{dim} V^{1}$.

Proof of Lemma. Choose $K$ and $f_{i}:\left(Z^{i}, \mathcal{M}^{i}\right) \rightarrow(S, \mathcal{L})=\left(\operatorname{Spec} O_{K}, \mathcal{L}_{K}\right)$ for $\left(V^{i}, \bar{V}^{i}\right)$ as in 2.2 so that $\psi_{i}$ come from morphisms $\psi_{i}$ : $\left(Z^{i+1}\right.$, $\left.\mathcal{M}^{i+1}\right) \rightarrow\left(Z^{i}, \mathcal{M}^{i}\right)$ over $(S, \mathcal{L})$. Since $R \Gamma\left(Z_{1}^{i}, \Omega_{\left(Z^{i}, \mathcal{M}^{i}\right)_{1} /(S, \mathcal{L})_{1}}^{a}\right)=R \Gamma\left(Z^{i}\right.$, $\left.\left.\Omega_{\left(Z^{i}, \mathcal{M}^{i}\right) /(S, \mathcal{L})}^{a}\right)\right) \otimes_{O_{K}}^{L}\left(O_{K} / p\right)$ and $\psi_{i}$ is $p$-negligible, we know that the $\psi_{i}^{*}$ morphisms $R \Gamma\left(Z_{1}^{i}, \Omega_{\left(Z^{i}, \mathcal{M}^{i}\right)_{1} /(S, \mathcal{L})_{1}}^{a}\right) \rightarrow R \Gamma\left(Z_{1}^{i+1}, \Omega_{\left(Z^{i+1}, \mathcal{M}^{i+1}\right)_{1} /(S, \mathcal{L})_{1}}^{a}\right), a>0$, and $\tau_{>0} R \Gamma\left(Z_{1}^{i}, \mathcal{O}_{Z_{1}^{i}}\right) \rightarrow \tau_{>0} R \Gamma\left(Z_{1}^{i+1}, \mathcal{O}_{Z_{1}^{i+1}}\right)$ vanish as maps in the derived category of $O_{K} / p$-modules. ${ }^{43}$ We are done now by (1.10.1), since the span of the conjugate filtration on the source is $\left[0, \operatorname{dim} V^{1}\right]$.

2.4. For $X \in \mathcal{V} a r_{\bar{K}}$ set $R \Gamma_{\text {crys }}(X):=R \Gamma\left(X_{\mathrm{h}}, \mathcal{A}_{\text {crys }}\right)$. This is an $\mathrm{E}_{\infty} \mathrm{A}_{\text {crys }}-$ algebra equipped with the Frobenius action $\varphi$. The Galois group $\operatorname{Gal}\left(\bar{K} / K_{0}\right)$ acts on $\mathcal{V}_{\bar{K}}$, and it acts on $X \mapsto R \Gamma_{\text {crys }}(X)$ by transport of structure. In particular, if $X$ is defined over an extension $K \subset \bar{K}$ of $K_{0}, X=X_{K} \otimes_{K}$

\footnotetext{
${ }^{42}$ To see $\mathcal{A}_{\text {crys }}$ explicitly, one computes the complex of presheaves $(V, \bar{V}) \mapsto$ $R \Gamma_{\text {crys }}(V, \bar{V})$ using Godement's resolution, sheafifies it for the h-topology on $\mathcal{V} a r_{\bar{K}}^{\mathrm{ss}}$, and views the result as a complex of h-sheaves on $\mathcal{V} a r_{\bar{K}}$.

${ }^{43}$ For $O_{\bar{K}} / p$ is faithfully $O_{K} / p$-flat and $R \Gamma\left(Z^{i}, \Omega_{\left(Z^{i}, \mathcal{M}^{i}\right) /(S, \mathcal{L})}^{a}\right)$ are $O_{K} / p$-perfect complexes.
} 
$\bar{K}$, then $\operatorname{Gal}(\bar{K} / K)$ acts naturally on $R \Gamma_{\text {crys }}(X)$. We write $H_{\text {crys }}^{n}(X):=$ $H^{n} R \Gamma_{\text {crys }}(X)$.

By the theorem in 2.3, the proposition in 2.1 provides canonical quasiisomorphisms of $\mathrm{E}_{\infty} \mathrm{A}_{\mathrm{crys} n}$-algebras

$$
R \Gamma_{\text {crys }}(X) \otimes^{L} \mathbb{Z} / p^{n} \stackrel{\sim}{\rightarrow} R \Gamma\left(X_{\text {ét }}, \mathbb{Z} / p^{n}\right) \otimes_{\mathbb{Z} / p^{n}}^{L} \mathrm{~A}_{\text {crys } n}
$$

and, since $A_{\text {crys }} \stackrel{\sim}{\rightarrow} A_{\text {crys }} \widehat{\otimes} \mathbb{Z}_{p}$, a canonical quasi-isomorphism of $\mathrm{E}_{\infty} \mathrm{A}_{\text {crys }^{-}}$ algebras

$$
R \Gamma_{\text {crys }}(X) \widehat{\otimes} \mathbb{Z}_{p} \stackrel{\sim}{\rightarrow} R \Gamma_{\text {ét }}\left(X, \mathbb{Z}_{p}\right) \otimes_{\mathbb{Z}_{p}} \mathrm{~A}_{\text {crys }}
$$

2.5. To control $R \Gamma_{\text {crys }}(X) \otimes \mathbb{Q}$, we use its connection with the de Rham cohomology provided by the Hyodo-Kato theory:

For an ss-pair $(V, \bar{V})$ over $\bar{K}$, set $R \Gamma_{\mathrm{HK}}(V, \bar{V}):=R \Gamma_{\mathrm{HK}}\left((V, \bar{V})_{1}\right)$, see Remark (ii) in 1.18. By loc. cit., one has natural isomorphisms $\iota_{\text {crys }}$ : $R \Gamma_{\mathrm{HK}}(V, \bar{V})_{\mathrm{B}_{\text {crys }}^{+}}^{\tau} \stackrel{\sim}{\rightarrow} R \Gamma_{\text {crys }}(V, \bar{V}) \otimes \mathbb{Q}, \iota_{\mathrm{dR}}: R \Gamma_{\mathrm{HK}}(V, \bar{V})_{\bar{K}}^{\tau} \stackrel{\sim}{\rightarrow} R \Gamma_{\mathrm{dR}}\left(V_{\bar{K}}, \bar{V}_{\bar{K}}\right)$. Let $\mathcal{A}_{\mathrm{HK}}$ be h-sheafification of the presheaf $(V, \bar{V}) \mapsto R \Gamma_{\mathrm{HK}}(V, \bar{V})$ on $\mathcal{V} a r_{\bar{K}}^{\mathrm{ss}}$; this is an h-sheaf of $\mathrm{E}_{\infty} K_{0}^{\mathrm{nr}}$-algebras on $\mathcal{V} a r_{\bar{K}}$ equipped with $\varphi$-action and locally nilpotent derivation $N$ such that $N \varphi=p \varphi N$. For $X \in \mathcal{V}_{a r} \bar{K}_{\bar{K}}$ set $R \Gamma_{\mathrm{HK}}(X):=R \Gamma\left(X_{\mathrm{h}}, \mathcal{A}_{\mathrm{HK}}\right), H_{\mathrm{HK}}^{n}(X):=R \Gamma_{\mathrm{HK}}(X)$. We get canonical quasiisomorphisms ${ }^{44}$

$$
\iota_{\text {crys }}: R \Gamma_{\mathrm{HK}}(X)_{\mathrm{B}_{\text {crys }}^{+}}^{\tau} \stackrel{\sim}{\rightarrow} R \Gamma_{\text {crys }}(X) \otimes \mathbb{Q}, \quad \iota_{\mathrm{dR}}: R \Gamma_{\mathrm{HK}}(X)_{\bar{K}}^{\tau} \stackrel{\sim}{\rightarrow} R \Gamma_{\mathrm{dR}}(X)
$$

compatible with the $\operatorname{Gal}\left(\bar{K} / K_{0}\right)$-action; here ${ }_{\mathrm{B}_{\text {crys }}^{+}}^{\tau},{ }_{\bar{K}}^{\tau}$ are the crystalline and de Rham Fontaine-Hyodo-Kato twists (they commute with the passage to h-sheafification and $R \Gamma)$.

Proposition. (i) For any $(V, \bar{V}) \in \mathcal{V}$ ar $r_{\bar{K}}^{s s}$ the canonical maps $R \Gamma_{\text {crys }}(V, \bar{V}) \otimes$ $\mathbb{Q} \rightarrow R \Gamma_{\text {crys }}(V) \otimes \mathbb{Q}, R \Gamma_{H K}(V, \bar{V}) \rightarrow R \Gamma_{H K}(V)$ are quasi-isomorphisms.

(ii) For every $X \in \mathcal{V}_{\text {ar }} \bar{K}_{\bar{K}}$ the cohomology groups $H_{\text {crys }}^{n}(X) \otimes \mathbb{Q}, H_{H K}^{n}(X)$ are free $B_{\text {crys }}^{+}$-modules, resp. $K_{0}^{n r}$-vector spaces, of rank equal to $\operatorname{dim} H_{e ́ t}^{n}\left(X, \mathbb{Q}_{p}\right)$. The same is true for the relative cohomology groups for a map of varieties.

Proof. (i) The map $R \Gamma_{\mathrm{dR}}(V, \bar{V}) \rightarrow R \Gamma_{\mathrm{dR}}(V)$ is a quasi-isomorphism by usual mixed Hodge theory (see (i) of the proposition in [B] 3.4). Using $\iota_{\mathrm{dR}}$, we see that $R \Gamma_{\mathrm{HK}}(V, \bar{V}) \stackrel{\sim}{\rightarrow} R \Gamma_{\mathrm{HK}}(V)$; applying $\iota_{\text {crys }}$, we get $R \Gamma_{\text {crys }}(V, \bar{V}) \otimes \mathbb{Q} \stackrel{\sim}{\rightarrow}$ $R \Gamma_{\text {crys }}(V) \otimes \mathbb{Q}$.

\footnotetext{
${ }^{44}$ Here $R \Gamma_{\mathrm{dR}}$ is Deligne's version of the de Rham cohomology, see [B] 3.4.
} 
(ii) One has $\operatorname{dim}_{\bar{K}} H_{\mathrm{dR}}^{n}(X)=\operatorname{dim} H_{\text {ét }}^{n}\left(X, \mathbb{Q}_{p}\right)$ (see (ii) of the proposition in [B] 3.4). Now use $\iota_{\mathrm{dR}}$ and $\iota_{\text {crys }}$ as in (i). ${ }^{45}$ Ditto for the relative cohomology.

Exercise. Assertion (ii) remains valid for any finite diagram of varieties.

2.6. Assertion (i) of the above proposition can be generalized as follows. Let $(Z, \mathcal{M}) /(S, \mathcal{L})$ be as in Example at the end of 1.16 with $K$ a subfield of $\bar{K}$. Denote by $\left(Z^{-}, \mathcal{M}^{-}\right)$the pullback of $(Z, \mathcal{M})$ by $(\bar{S}, \overline{\mathcal{L}}) \rightarrow(S, \mathcal{L})$ (see 1.17 , 1.18 for the notation), by $\left(\bar{X}, \mathcal{M}_{\bar{X}}\right)$ the generic fiber of $\left(Z^{-}, \mathcal{M}^{-}\right)$, i.e., the pullback of $(Z, \mathcal{M})$ to $\bar{K}$, and by $X \subset \bar{X}$ the open subset of triviality of the $\log$ structure. Let us define canonical maps

$$
R \Gamma_{\text {crys }}\left(Z^{-}, \mathcal{M}^{-}\right) \rightarrow R \Gamma_{\text {crys }}(X), \quad R \Gamma_{\mathrm{HK}}\left(Z_{1}^{0}, \mathcal{M}_{1}^{0}\right) \rightarrow R \Gamma_{\mathrm{HK}}(X) .
$$

Consider the arithmetic pair $\left(X, Z^{-}\right)$over $\bar{K}$, and pick any h-hypercovering $(V ., \bar{V}$.$) of \left(X, Z^{-}\right)$by ss-pairs (see [B] $\left.\S 2\right)$. One has an evident map of $\log$ schemes $^{46}\left(X, Z^{-}\right) \rightarrow\left(Z^{-}, \mathcal{M}^{-}\right)$, so $(V ., \bar{V}$. $)$ is a simplicial log scheme over $\left(Z^{-}, \mathcal{M}^{-}\right)$. Our maps are compositions $R \Gamma_{\text {crys }}\left(Z^{-}, \mathcal{M}^{-}\right) \rightarrow R \Gamma_{\text {crys }}(V ., \bar{V}.) \rightarrow$ $R \Gamma_{\text {crys }}(V) \stackrel{\leftarrow}{\leftarrow} R \Gamma_{\text {crys }}(X)$ and $R \Gamma_{\mathrm{HK}}\left(Z_{1}^{0}, \mathcal{M}_{1}^{0}\right) \rightarrow R \Gamma_{\mathrm{HK}}\left(Z^{-}, \mathcal{M}^{-}\right) \rightarrow$ $R \Gamma_{\mathrm{HK}}(V ., \bar{V}.) \rightarrow R \Gamma_{\mathrm{HK}}(V.) \stackrel{\leftarrow}{\leftarrow} R \Gamma_{\mathrm{HK}}(X)$.

Suppose the sheaf of groups $\mathcal{M}_{\bar{X}}^{\text {gr }} / \mathcal{O}_{\bar{X}}^{\times}$has trivial torsion (e.g. $\mathcal{M}_{\bar{X}}$ is saturated).

Proposition. The maps of (2.6.1) yield quasi-isomorphisms $R \Gamma_{\text {crys }}\left(Z^{-}\right.$, $\left.\mathcal{M}^{-}\right) \otimes \mathbb{Q} \stackrel{\sim}{\rightarrow} R \Gamma_{\text {crys }}(X) \otimes \mathbb{Q}, R \Gamma_{H K}\left(Z_{1}^{0}, \mathcal{M}_{1}^{0}\right) \otimes_{K_{0}} K_{0}^{n r} \stackrel{\sim}{\rightarrow} R \Gamma_{H K}(X)$.

Proof. Our maps are compatible with identifications $\iota_{\text {crys }}$ of (1.18.2) and (2.5.1), so it suffices to check the claim for $R \Gamma_{\mathrm{HK}}$. Due to isomorphisms $\iota_{\mathrm{dR}}$ of (1.16.2) and (2.5.1), it is enough to show that the restriction $R \Gamma\left(\bar{X}, \Omega_{\left(\bar{X}, \mathcal{M}_{\bar{X}}\right) / \bar{K}}\right) \rightarrow R \Gamma\left(X, \Omega_{X / \bar{K}}\right)=R \Gamma_{\mathrm{dR}}(X)$ is a quasi-isomorphism, which follows from Ogus' theorem in 1.19.

\section{The Fontaine-Jannsen conjecture}

3.1. For $X \in \mathcal{V} a r_{\bar{K}}$ we define the crystalline period map

$$
\rho_{\text {crys }}: R \Gamma_{\text {crys }}(X) \rightarrow R \Gamma_{\text {ét }}\left(X, \mathbb{Z}_{p}\right) \otimes_{\mathbb{Z}_{p}} \mathrm{~A}_{\text {crys }}
$$

${ }^{45} H_{\text {crys }}^{n}(X) \otimes \mathbb{Q}$ is a free $\mathrm{B}_{\text {crys }}^{+}-$module being a twist of $H_{\mathrm{HK}}^{n}(X) \otimes K_{0}^{\text {nr }} \mathrm{B}_{\text {crys }}^{+}$by a trivial torsor.

${ }^{46}$ We view pairs as log schemes as in [B] 3.2. 
as the composition of the evident map $R \Gamma_{\text {crys }}(X) \rightarrow R \Gamma_{\text {crys }}(X) \widehat{\otimes} \mathbb{Z}_{p}$ with identification (2.4.2). Composing $\rho_{\text {crys }} \otimes \mathbb{Q}$ with isomorphism $\iota_{\text {crys }}$ from (2.5.1), we get

$$
\rho_{\mathrm{HK}}: R \Gamma_{\mathrm{HK}}(X)_{\mathrm{B}_{\text {crys }}^{+}}^{\tau} \rightarrow R \Gamma_{\text {ét }}\left(X, \mathbb{Q}_{p}\right) \otimes_{\mathbb{Q}_{p}} \mathrm{~B}_{\text {crys }}^{+}
$$

These are morphisms of $\mathrm{E}_{\infty} \mathrm{A}_{\text {crys }^{-}}$and $\mathrm{B}_{\text {crys }}^{+}$-algebras equipped with the Frobenius action $\varphi$ (it acts on the target via the second factor). The Galois $\operatorname{group} \operatorname{Gal}\left(\bar{K} / K_{0}\right)$ acts on $\mathcal{V}_{a r}$ and all the functors; $\rho_{\text {crys }}, \rho_{\mathrm{HK}}$ are compatible with this action. Thus if $X$ is defined over an extension $K \subset \bar{K}$ of $K_{0}$, $X=X_{K} \otimes_{K} \bar{K}$, then $\operatorname{Gal}(\bar{K} / K)$ acts on both terms of (3.1.1), (3.1.2) and $\rho_{\text {crys }}, \rho_{\text {HK }}$ commute with the Galois action.

3.2. We use the notation from 1.17. Below $\mathbb{Q}_{p} / \mathbb{Z}_{p}(1)$ is the subgroup of $p^{\infty}$-roots of 1 in $O_{\bar{K}}^{\times}$, so $\mathbb{Q}_{p}(1)$ is projective limit of the system $\ldots \stackrel{p}{\rightarrow}$ $\mathbb{Q}_{p} / \mathbb{Z}_{p}(1) \stackrel{p}{\rightarrow} \mathbb{Q}_{p} / \mathbb{Z}_{p}(1)$. Thus one has an embedding $\mathbb{Q}_{p}(1) \hookrightarrow \mathcal{L}_{\varphi},\left(\varepsilon^{(n)}\right) \mapsto$ $\left(\varepsilon^{(n)} \bmod p O_{\bar{K}}\right) \in \mathcal{L}_{\varphi}$, hence a canonical embedding $\mathbb{Q}_{p}(1) \hookrightarrow \mathrm{A}_{\text {crys }}^{\times}$. The image of $\mathbb{Z}_{p}(1)$ lies in $\left(1+J_{\text {crys }}\right)^{\times}$; applying log: $\left(1+J_{\text {crys }}\right)^{\times} \rightarrow J_{\text {crys }}$, we get $l: \mathbb{Z}_{p}(1) \hookrightarrow J_{\text {crys }} \subset \mathrm{A}_{\text {crys }}$. As in [F1] 2.3.4, set $\mathrm{B}_{\text {crys }}:=\mathrm{A}_{\text {crys }}\left[l(t)^{-1}\right]$, where $t$ is a generator of $\mathbb{Z}_{p}(1)$. Inverting $l(t)$ implies inverting $p,{ }^{47}$ so $\mathrm{B}_{\text {crys }} \supset \mathrm{B}_{\text {crys }}^{+}$.

Theorem. The $B_{\text {crys }}$-linear extensions of $\rho_{\text {crys }}$ and $\rho_{H K}$ are quasiisomorphisms: for any $X \in \mathcal{V}_{\bar{K}}$ one has $\rho_{\text {crys }}: R \Gamma_{\text {crys }}(X) \otimes_{A_{\text {crys }}} B_{\text {crys }} \stackrel{\sim}{\rightarrow}$ $R \Gamma_{e ́ t}\left(X, \mathbb{Q}_{p}\right) \otimes B_{\text {crys }}, \rho_{H K}: R \Gamma_{H K}(X)_{B_{\text {crys }}}^{\tau} \stackrel{\sim}{\rightarrow} R \Gamma_{e ́ t}\left(X, \mathbb{Q}_{p}\right) \otimes B_{\text {crys }}$.

Proof. It is very similar to that of the $\rho_{\mathrm{dR}}$ counterpart in $[\mathrm{B}] 3.6$ : there is a calculation for circle (Lemma below), the rest comes by a general functoriality argument.

(a) The case of $X=\mathbb{G}_{m}=\mathbb{P}^{1} \backslash\{0, \infty\}$ : Let $t$ be the standard parameter on $\mathbb{G}_{m}$, and $\overline{\mathbb{G}}_{m}$ be $\mathbb{P}^{1}$ viewed as a $\mathbb{G}_{m}$-equivariant compactification of $\mathbb{G}_{m}$. So $\left(\mathbb{G}_{m \bar{K}}, \overline{\mathbb{G}}_{m \bar{S}}\right)$ is an ss-pair over $\bar{K}$; denote the corresponding log $\bar{S}$-scheme by $Y_{\bar{S}}$. Its $\log$ structure is generated by $\overline{\mathcal{L}}$ and $t$. Let $Y_{\text {crys }}$ be $\overline{\mathbb{G}}_{m E_{\text {crys }}}$ equipped with the log structure on $\overline{\mathbb{G}}_{m E_{\text {crys }}}$ generated by $t$ and $\mathcal{L}_{\text {crys }}$.

By (ii) of the proposition in 2.5, it suffices to consider the group $H^{1}$. Consider the canonical map $R \Gamma_{\text {crys }}\left(Y_{\bar{S}}\right) \rightarrow R \Gamma_{\text {crys }}\left(\mathbb{G}_{m \bar{K}}\right)$. Since $Y_{\text {crys }}$ is a pd-smooth object of $\left(Y_{\bar{S}_{1}} /\left(E_{\text {crys }}, \mathcal{L}_{\text {crys }}\right)\right)_{\text {crys }}$, the $R \Gamma$ of its de Rham complex equals $R \Gamma_{\text {crys }}\left(Y_{\bar{S}}\right)$ (see $\left.(1.8 .1),(1.18 .1)\right)$, so $H_{\text {crys }}^{1}\left(Y_{\bar{S}}\right)$ is a free $\mathrm{A}_{\text {crys }}$-module generated by $d \log t$. By (i) of the proposition in $2.5, H_{\text {crys }}^{1}\left(\mathbb{G}_{m \bar{K}}\right) \otimes \mathbb{Q}$ is a free $\mathrm{B}_{\text {crys }}^{+}-$module generated by the image of $d \log t$. Let $\kappa$ be the canonical

${ }^{47}$ For the pd structure on $J_{\text {crys }}$ provides $p^{-1} l(t)^{p} \in J_{\text {crys }}$. 
generator of $H_{\text {ét }}^{1}\left(\mathbb{G}_{m \bar{K}}, \mathbb{Z}_{p}(1)\right)=H_{\text {ét }}^{1}\left(\mathbb{G}_{m \bar{K}}, \mathbb{Z}_{p}\right) \otimes \mathbb{Z}_{p}(1) ;$ applying $l: \mathbb{Z}_{p}(1) \hookrightarrow$ $\mathrm{A}_{\text {crys }}$, we get $l(\kappa) \in H_{\text {ét }}^{1}\left(\mathbb{G}_{m \bar{K}}, \mathbb{Z}_{p}\right) \otimes \mathrm{A}_{\text {crys. }}$. It remains to prove:

Lemma. One has $\rho_{\text {crys }}(d \log t)=l(\kappa)$.

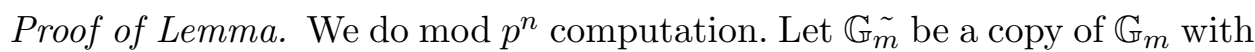
parameter $\tilde{t}$, and $\pi: \mathbb{G} \tilde{m} \rightarrow \mathbb{G}_{m}$ be the map $\pi^{*}(t)=\tilde{t}^{p^{n}}$. Then $\mathbb{G}_{m} \tilde{\bar{K}} / \mathbb{G}_{m} \bar{K}$ is a $\mathbb{Z} / p^{n}(1)$-torsor, and $\kappa_{n} \in H^{1}\left(\mathbb{G}_{m \bar{K} \text { ét }}, \mathbb{Z} / p^{n}(1)\right)$ is its class. The corresponding Cech hypercovering is the twist of $\mathbb{G}_{m} \bar{K}_{\bar{K}}$ by the universal $\mathbb{Z} / p^{n}(1)$-torsor $\mathfrak{t}$ over the simplicial classifying space $B$. of $\mathbb{Z} / p^{n}(1)$, and $\kappa_{n}$ comes from an evident 1-cocycle on $B$. Now $\pi$ extends to an h-covering $\left(\mathbb{G}_{\tilde{m}} \bar{K}, \overline{\mathbb{G}}_{m} \bar{S}\right) \rightarrow$ $\left(\mathbb{G}_{m \bar{K}}, \overline{\mathbb{G}}_{m \bar{S}}\right)$ of ss-pairs; its t-twist $\pi .:\left(\mathbb{G}_{m} \tilde{\bar{K}}, \overline{\mathbb{G}}_{m} \bar{S}_{\bar{S}}\right) . \rightarrow\left(\mathbb{G}_{m \bar{K}}, \overline{\mathbb{G}}_{m \bar{S}}\right)$ is an h-hypercovering in $\mathcal{V} r_{\bar{K}}^{\mathrm{ss}}$. Changing the notation, we have $\pi: Y_{\tilde{S}} \rightarrow Y_{\bar{S}}$ and $\pi$. $: Y \tilde{\bar{S}}_{\text {. }} \rightarrow Y_{\bar{S}}$. We want to check that $\pi_{\text {crys }}^{*}: R \Gamma_{\text {crys }}\left(Y_{\bar{S}}\right)_{(n)} \rightarrow R \Gamma_{\text {crys }}\left(Y_{\bar{S}}\right)_{(n)}$ sends $d \log t$ to $l\left(\kappa_{n}\right)$. To do this, we extend $\pi_{1}$. to a map of simplicial pdsmooth thickenings $P^{r} \rightarrow P$. over $E_{\text {crys } n}$.

Set $P:=Y_{\text {crys } n}, \mathcal{G}:=\mathbb{G}_{m E_{\text {crys } n}}\left(:=\right.$ the pd-envelope of $\mathbb{G}_{m}$ at 1 , see $1.2)$. Then $\mathcal{G}$ acts on $P$ through $\mathcal{G} \rightarrow \mathbb{G}_{m}$. Our $P$. is the twist of $P$ by the universal $\mathcal{G}$-torsor over the simplicial classifying space $B_{\mathcal{G}} .{ }^{48}$ Consider now the ${ }^{\sim}$-copies $P^{\sim}$ and $\mathcal{G}^{\sim}$. Together with the $\mathcal{G}^{\sim}$-action, $P^{\sim}$ carries an action of $p^{-n} \mathbb{Z}_{p}$ via the composition $p^{-n} \mathbb{Z}_{p}(1) \subset \mathbb{Q}_{p}(1) \hookrightarrow \mathrm{A}_{\text {crys }}^{\times} \rightarrow \mathrm{A}_{\text {crys } n}^{\times}=$ $\mathbb{G}_{m} \tilde{m}\left(E_{\text {crys } n}\right)$, where $\hookrightarrow$ is the canonical embedding. Its restriction to $\mathbb{Z}_{p}(1)$ lands in $\left(1+J_{\operatorname{crys} n}\right)^{\times}$, i.e., we have a homomorphism $\alpha: \mathbb{Z}_{p}(1) \rightarrow \mathcal{G}^{\sim}\left(E_{\operatorname{crys} n}\right)$. Both actions combine into an action of the group pd-scheme $\mathcal{G}^{+}$which is an extension of $\left(p^{-n} \mathbb{Z}_{p} / \mathbb{Z}_{p}\right)(1)_{E_{\text {crys } n}}$ by $\mathcal{G}^{\sim}$ defined as the pushout of $0 \rightarrow$ $\mathbb{Z}_{p}(1) \rightarrow p^{-n} \mathbb{Z}_{p}(1) \rightarrow\left(p^{-n} \mathbb{Z}_{p} / \mathbb{Z}_{p}\right)(1) \rightarrow 0$ by $\alpha$. Our $P^{\sim}$. is the twist of $P^{\sim}$ by the universal $\mathcal{G}^{+}$-torsor over the simplicial classifyling space $B_{\mathcal{G}^{+}}$. Extension $\mathcal{G}^{+}$splits over $\bar{S}_{1}$ since $\alpha$ vanishes at $\bar{S}_{1} \subset E_{\text {crys } n}$, so we get

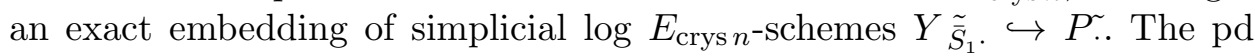
structure on $\mathcal{G}^{\sim}$ provides a pd structure on its ideal. Finally, the projection $\pi: P^{\sim} \rightarrow P$ and an evident "multiplication by $p^{n}$ " morphism $\pi^{+}: \mathcal{G}^{+} \rightarrow \mathcal{G}$ yield a map $\pi_{P .}: P^{\sim} \rightarrow P$. of pd-thickenings that extends $\pi_{1}$.

The pd-thickenings $P_{i}$ and $P_{i}^{\sim}$ are pd-smooth over $E_{\operatorname{crys} n}$, so, by (1.8.1), (1.18.1), the map $\pi_{\text {crys }}^{*}: R \Gamma_{\text {crys }}\left(Y_{\bar{S}}\right)_{(n)} \rightarrow R \Gamma_{\text {crys }}\left(Y \tilde{S}_{\bar{S}}\right)_{(n)}$ coincides with the pullback map $\pi_{P .}^{*}: R \Gamma\left(P ., \Omega_{P .}\right) \rightarrow R \Gamma\left(P^{\sim}, \Omega_{P^{\sim}}\right)$ between the total de Rham complexes; here $\Omega_{?}:=\Omega_{? /\left(E_{\text {crys }}, \mathcal{L}_{\text {crys }}\right)_{n}}$. Now $d \log t \in \Gamma\left(P_{0}, \Omega_{P_{0}}^{1}\right)$ extends to a total 1 -cocycle in $\Gamma\left(P ., \Omega_{P}\right)$ by adding the component $\log \chi \in$ $\Gamma\left(P_{1}, \mathcal{O}_{P_{1}}\right)$ which comes from the evident $\mathcal{G}$-valued 1-cocycle $\chi$ on $B_{\mathcal{G}}$. One has $\pi_{P 0}^{*}(d \log t)=p^{n} d \log \tilde{t}=0$, and $\pi_{P 1}^{*}(\log \chi)$ comes from the 1-cocycle $\log \left(\chi \pi^{+}\right)$on $B_{\mathcal{G}}$, which is $l\left(\kappa_{n}\right)$, q.e.d.

\footnotetext{
${ }^{48} P$. is equal to the simplicial object $P_{*}$ of $\left(Y_{\bar{S}_{1}} /\left(E_{\text {crys }}, \mathcal{L}_{\text {crys }}\right)_{n}\right)_{\text {crys }}$ from 1.6.
} 
(b) Compatibility of $\rho_{\text {crys }}$ with the Gysin maps for codimension 1 closed embeddings of smooth varieties: Let $i: Y \hookrightarrow X$ be such an embedding. For any cohomology theory $R \Gamma_{\text {? }}$ we deal with, consider the cohomology with supports $R \Gamma_{? Y}(X):=\mathcal{C}$ one $\left(R \Gamma_{?}(X) \rightarrow R \Gamma_{?}(X \backslash Y)\right)[-1]$. Recall a definition of the Gysin isomorphism $i_{*}: R \Gamma_{\text {? }}(Y) \stackrel{\sim}{\rightarrow} R \Gamma_{\text {? }}(X)[2]$. (Here the Tate twist (1) in the target is canceled due to specifics of the cohomology theories we deal with, see below.)

Let $\mathcal{L}$ be the normal line bundle, $i_{0}: Y \hookrightarrow \mathcal{L}$ its zero section. There is a canonical identification $\eta: R \Gamma_{\text {?Y }}(\mathcal{L}) \stackrel{\sim}{\rightarrow} R \Gamma_{\text {?Y }}(X)$ defined using the deformation to normal cone construction. Namely, we have the diagram

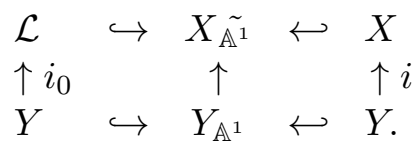

Here $Y_{\mathbb{A}^{1}}=Y \times \mathbb{A}^{1}, X_{\mathbb{A}^{1}}$ is $X \times \mathbb{A}^{1}$ with $Y \times\{0\}$ blown up, the bottom embeddings are $y \mapsto(y, 0),(y, 1)$. The arrows $R \Gamma_{\text {?Y }}(\mathcal{L}) \leftarrow R \Gamma_{\text {? } Y_{\mathbb{A}^{1}}}\left(X_{\mathbb{A}^{1}}\right) \rightarrow$ $R \Gamma_{\text {?Y }}(X)$ are quasi-isomorphisms (this is standard for $R \Gamma_{\text {ét }}, R \Gamma_{\mathrm{dR}} ;^{49}$ the assertion for $R \Gamma_{\mathrm{HK}}$ and $R \Gamma_{\text {crys }} \otimes \mathbb{Q}$ is deduced from that for $R \Gamma_{\mathrm{dR}}$ using (2.5.1)). Their composition is $\eta$.

One has $i_{*}:=\eta i_{0 *}$, so it suffices to define $i_{0 *}$. The projection $\mathcal{L} \rightarrow$ $Y$ makes $R \Gamma_{\text {? } Y}(\mathcal{L})$ an $R \Gamma_{?}(Y)$-module, and $i_{0 *}$ is a morphism of $R \Gamma_{?}(Y)$ modules. Thus to define $i_{0 *}$, we need to specify the orientation class $i_{0 *}(1) \in$ $H_{? Y}^{2}(\mathcal{L}) \stackrel{\sim}{\leftarrow} \tau_{\leq 0}\left(R \Gamma_{? Y}(\mathcal{L})[2]\right)$ (here $\tilde{\leftarrow}$ holds due to (ii) of the proposition in 2.5). If $Y$ is connected, then $H_{? Y}^{2}(\mathcal{L})$ is a free module of rank 1 (see loc. cit.), so, localizing $Y$, we can assume that $\mathcal{L}$ is trivialized. Then $H_{\text {?Y }}^{2}(\mathcal{L}) \leftarrow$ $H_{?}^{1}\left(\mathbb{G}_{m \bar{K}}\right)$, and we define $i_{0 *}(1)$ to be $d \log t$ for $R \Gamma_{\mathrm{dR}}, R \Gamma_{\mathrm{HK}}, R \Gamma_{\text {crys }} \otimes \mathbb{Q}$, and $l(\kappa)$ for $R \Gamma_{\text {ét }}\left(\cdot, \mathbb{Q}_{p}\right) \otimes \mathrm{B}_{\text {crys }}$ (see (a) for the notation). We have defined $i_{0 *}$, hence $i_{*}$. It is an isomorphism (standard for $R \Gamma_{\text {ét }}, R \Gamma_{\mathrm{dR}}$; the assertion for $R \Gamma_{\mathrm{HK}}$ and $R \Gamma_{\text {crys }} \otimes \mathbb{Q}$ is deduced from that for $R \Gamma_{\mathrm{dR}}$ using (2.5.1)). Our $i_{*}$ commutes with the maps of $(2.5 .1)$ and, by lemma in (a), with $\rho_{\text {crys }}$.

(c) The case of smooth projective $X$ : We can assume that $X$ is connected, $\operatorname{dim} X=d$. Then $H_{?}^{2 d}(X)$ is a free module of rank 1 (see (ii) of the proposition in 2.5), and the Poincaré duality pairing $H_{?}^{i}(X) \times H_{?}^{2 d-i}(X) \rightarrow H_{?}^{2 d}(X)$ is nondegenerate (standard for $R \Gamma_{\text {ét }}, R \Gamma_{\mathrm{dR}}$; the assertion for $R \Gamma_{\mathrm{HK}}$ and $R \Gamma_{\text {crys }} \otimes \mathbb{Q}$ is deduced from that for $R \Gamma_{\mathrm{dR}}$ using $\left.(2.5 .1)\right)$. Since $\rho_{\text {crys }}$ is a morphism of algebras, it is compatible with the Poincaré duality. This, together with (ii) of the proposition in 2.5 , implies that $\rho_{\text {crys }}: H_{\text {crys }}^{i}(X) \otimes \mathrm{B}_{\text {crys }} \rightarrow$

\footnotetext{
${ }^{49}$ Use purity to identify the arrows with $R \Gamma_{?}(Y) \leftarrow R \Gamma_{?}\left(Y_{\mathbb{A}^{1}}\right) \rightarrow R \Gamma_{?}(Y)$.
} 
$H_{\text {ét }}^{i}\left(X, \mathbb{Q}_{p}\right) \otimes \mathrm{B}_{\text {crys }}$ is an isomorphism for every $i$ if this is true for $i=2 d$. To check the latter assertion, consider the class $c_{?} \in H_{?}^{2}(X)$ of hyperplane section (defined using the corresponding Gysin map). Then $c_{\text {ét }}^{d}$ is a base in $H_{\text {ét }}^{2 d}\left(X, \mathbb{Q}_{p}\right) \otimes \mathrm{B}_{\text {crys }}, c_{\text {crys }}^{d}$ is a base in $H_{\text {crys }}^{2 d}(X) \otimes \mathbb{Q}$ (by $(2.5 .1)$, since $c_{\mathrm{dR}}^{d}$ is a base in $\left.H_{\mathrm{dR}}^{2 d}(X)\right)$. Now $\rho_{\text {crys }}\left(c_{\text {crys }}\right)=c_{\text {ét }}$ by $(\mathrm{b})$, and we are done.

(d) The case when $X$ is the complement to a strict normal crossings divisor in a smooth projective variety: Checked exactly as in [B] 3.6, by induction by the number of the components of the divisor using (c) and (b).

(e) The case of arbitrary $X$ : Checked exactly as in [B] 3.6, using an h-hypercovering of $X$ by varieties as in (d).

3.3. The theorem in 3.2 implies the Fontaine-Jannsen conjecture. To see this, we pull $\rho_{\mathrm{HK}}$ back to the Fontaine-Hyodo-Kato $\mathbb{G}_{a}$-torsor ${ }^{50}$ Spec $\mathrm{B}_{\text {st }}$ over Spec $\mathrm{B}_{\text {crys }}$ to trivialize the twist, cf. (1.18.4). We get a canonical quasiisomorphism of $\mathrm{B}_{\mathrm{st}}$-complexes

$$
\rho_{\mathrm{HK}}: R \Gamma_{\mathrm{HK}}(X) \otimes K_{0}^{\mathrm{nr}} \mathrm{B}_{\mathrm{st}} \stackrel{\sim}{\rightarrow} R \Gamma_{\text {ét }}\left(X, \mathbb{Q}_{p}\right) \otimes \mathrm{B}_{\mathrm{st}}
$$

compatible with the $(\varphi, N)$-action and with the $\operatorname{Gal}(\bar{K} / K)$-action on $\mathcal{V}_{a r}{ }_{\bar{K}}$. This is the identification asked for in $[\mathrm{F} 2] \S 6$.

Conjectures $\mathrm{C}_{\mathrm{pst}}, \mathrm{C}_{\mathrm{st}}$, and $\mathrm{C}_{\text {crys }}$ (see [F2] 6.2.1, 6.2.7, 6.1.4) come as follows:

(i) Suppose $X$ is defined over a finite extension $K$ of $K_{0}, K \subset \bar{K}$, so we have $X_{K}$ over $K$ and an identification $X=X_{K} \otimes_{K} \bar{K}$. We get the $\operatorname{Gal}(\bar{K} / K)$ action on $R \Gamma_{\mathrm{HK}}(X)$. By [F1] 4.2.4, $H_{\mathrm{HK}}^{n}(X)$ coincides with the subspace of those elements in $H_{\mathrm{HK}}^{n}(X) \otimes_{K_{0}^{\mathrm{nr}}} \mathrm{B}_{\mathrm{st}}$ whose stabilizers in $\operatorname{Gal}(\bar{K} / K)$ are open, hence, via (3.3.1), with the similar subspace of $H_{\text {ét }}^{n}\left(X, \mathbb{Q}_{p}\right) \otimes \mathrm{B}_{\mathrm{st}}$. This is conjecture $\mathrm{C}_{\mathrm{pst}}$.

(ii) Assume we are in the situation of 2.6. Then $R \Gamma_{\mathrm{HK}}\left(Z_{1}^{0}, \mathcal{M}_{1}^{0}\right) \otimes_{K_{0}} K_{0}^{\mathrm{nr}} \stackrel{\sim}{\rightarrow}$ $R \Gamma_{\mathrm{HK}}(X)$ by the proposition in loc. cit., so $\operatorname{Gal}(\bar{K} / K)$ acts trivially on $R \Gamma_{\mathrm{HK}}\left(Z_{1}^{0}, \mathcal{M}_{1}^{0}\right)$. We get $\rho_{\mathrm{HK}}: R \Gamma_{\mathrm{HK}}\left(Z_{1}^{0}, \mathcal{M}_{1}^{0}\right) \otimes_{K_{0}} \mathrm{~B}_{\mathrm{st}} \stackrel{\sim}{\rightarrow} R \Gamma_{\text {ét }}\left(X, \mathbb{Q}_{p}\right) \otimes_{\mathbb{Q}_{p}} \mathrm{~B}_{\mathrm{st}}$, which is conjecture $\mathrm{C}_{\text {st }}$.

(iii) Assume we are in the situation of 2.6, and $\left(Z_{1}^{0}, \mathcal{M}_{1}^{0}\right) /\left(S_{1}^{0}, \mathcal{L}_{1}^{0}\right)$ can be realized as the pullback of a log scheme $\left(Z_{1}^{0}, \mathcal{M}_{0}^{0}\right)$ over $S_{1}^{0}=\operatorname{Spec} k$ (with trivial $\log$ structure) by the tautological map $\left(S_{1}^{0}, \mathcal{L}_{1}^{0}\right) \rightarrow S_{1}^{0}$. E.g. this happens if $X$ is smooth proper and $X_{K}$ has smooth model $Z$ : then the $\log$ structure $\mathcal{M}_{0}^{0}$ is trivial. By the base change, $R \Gamma_{\text {crys }}\left(Z_{1}^{0}, \mathcal{M}_{0}^{0}\right) \otimes \mathbb{Q} \stackrel{\sim}{\rightarrow} R \Gamma_{\mathrm{HK}}\left(Z_{1}^{0}, \mathcal{M}_{1}^{0}\right)$, so $N$ acts trivially on the Hyodo-Kato cohomology, and $\rho_{\mathrm{HK}}$ from the theorem in 3.2 can be rewritten as $R \Gamma_{\text {crys }}\left(Z_{1}^{0}, \mathcal{M}_{0}^{0}\right) \otimes_{W(k)} \mathrm{B}_{\text {crys }} \stackrel{\sim}{\rightarrow} R \Gamma_{\text {ét }}\left(X, \mathbb{Q}_{p}\right) \otimes \mathrm{B}_{\text {crys }}$, which is conjecture $\mathrm{C}_{\text {crys }}$.

\footnotetext{
${ }^{50}$ Recall that $\mathrm{B}_{\mathrm{st}}:=\mathrm{B}_{\mathrm{st}}^{+} \otimes_{\mathrm{B}_{\text {crys }}^{+}} \mathrm{B}_{\text {crys }}$.
} 
3.4. In the rest of the section we show that the crystalline period map is compatible with its derived de Rham cousin $\rho_{\mathrm{dR}}$ from [B]:

Theorem-construction. For any $X \in \mathcal{V}_{a r}{ }_{\bar{K}}$ there is a canonical isomorphism

$$
R \Gamma_{d R}(X) \otimes_{\bar{K}} B_{d R}^{+} \stackrel{\sim}{\rightarrow} R \Gamma_{\text {crys }}(X) \otimes_{A_{\text {crys }}} B_{d R}^{+},
$$

compatible with the Galois action such that $\rho_{d R}$ from $[B] 3.5 .4$ is the composition of (3.4.1) with $\rho_{\text {crys }} \otimes B_{d R}^{+}: R \Gamma_{\text {crys }}(X) \otimes A_{\text {crys }} B_{d R}^{+} \rightarrow R \Gamma_{\text {ét }}\left(X, \mathbb{Q}_{p}\right) \otimes B_{d R}^{+}$. Proof. Let us fix notation. As in [B] 3.3, for any ss-pair $(V, \bar{V})$ over $\bar{K}$ we denote by $R \Gamma_{\mathrm{dR}}^{\natural}(V, \bar{V})$ its absolute derived de Rham complex $R \Gamma\left(\bar{V}, L \Omega_{(\hat{V}, \bar{V}) / W(k)}\right)$. Set $R \Gamma_{\mathrm{dR}}^{\natural}(V, \bar{V})_{n}:=R \Gamma_{\mathrm{dR}}^{\natural}(V, \bar{V}) \otimes \otimes^{L} \mathbb{Z} / p^{n} \stackrel{\sim}{\rightarrow}$ $R \Gamma\left(\bar{V}, L \Omega_{(\hat{V}, \bar{V})_{n} / W_{n}(k)}\right)$, where $\stackrel{\sim}{\rightarrow}$ is the base change identification, $R \Gamma_{\mathrm{dR}}^{\natural}(V$, $\bar{V}) \widehat{\otimes} \mathbb{Z}_{p}:=\operatorname{holim}_{n} R \Gamma_{\mathrm{dR}}^{\natural}(V, \bar{V})_{n}, R \Gamma_{\mathrm{dR}}^{\natural}(V, \bar{V}) \widehat{\otimes} \mathbb{Q}_{p}:=\left(R \Gamma_{\mathrm{dR}}^{\natural}(V, \bar{V}) \widehat{\otimes} \mathbb{Z}_{p}\right) \otimes \mathbb{Q}$. These are $F$-filtered $\mathrm{E}_{\infty}$ algebras, where "F-filtered" means that we view them as mere projective systems of quotients modulo the terms of Hodge filtration $F^{m}$. Below we consider the homotopy $F$-completions of these complexes, which we denote by $\lim _{F}$. So we have $\lim _{F} R \Gamma_{\mathrm{dR}}^{\natural}(V, \bar{V})_{n}=$ $\operatorname{holim}_{m}\left(R \Gamma_{\mathrm{dR}}^{\natural}(V, \bar{V})_{n} / F^{m}\right), \lim _{F} R \Gamma_{\mathrm{dR}}^{\natural}(V, \bar{V}) \widehat{\otimes} \mathbb{Z}_{p}:=\operatorname{holim}_{m}\left(R \Gamma_{\mathrm{dR}}^{\natural}(V, \bar{V}) \widehat{\otimes} \mathbb{Z}_{p} /\right.$ $\left.F^{m}\right)=\operatorname{holim}_{m, n}\left(R \Gamma_{\mathrm{dR}}^{\natural}(V, \bar{V})_{n} / F^{m}\right)$, etc.

Recall that $\mathrm{A}_{\mathrm{dR}}=R \Gamma_{\mathrm{dR}}^{\natural}\left(\operatorname{Spec}\left(\bar{K}, O_{\bar{K}}\right)\right)$ (see the lemma in [B] 3.2). The corresponding $F$-filtered algebras $\mathrm{A}_{\mathrm{dR} n}, \mathrm{~A}_{\mathrm{dR}} \widehat{\otimes} \mathbb{Z}_{p}, \mathrm{~A}_{\mathrm{dR}} \widehat{\otimes} \mathbb{Q}_{p}$ are acyclic in degrees $\neq 0$ and their projections $\cdot / F^{m+1} \rightarrow \cdot / F^{m}$ are surjective (see [B] $1.4,1.5)$. Thus $\mathrm{A}_{\mathrm{dR} n}^{\diamond}:=\lim _{F} \mathrm{~A}_{\mathrm{dR} n}$ equals $\varliminf_{m} H^{0}\left(\mathrm{~A}_{\mathrm{dR} n} / F^{m}\right), \mathrm{A}_{\mathrm{dR}}^{\diamond}:=$ $\lim _{F}\left(\mathrm{~A}_{\mathrm{dR}} \widehat{\otimes} \mathbb{Z}_{p}\right)$ equals $\lim _{m} H^{0}\left(\mathrm{~A}_{\mathrm{dR}} \widehat{\otimes} \mathbb{Z}_{p} / F^{m}\right),{ }^{51}$ and $\lim _{F}\left(\mathrm{~A}_{\mathrm{dR}} \widehat{\otimes} \mathbb{Q}_{p}\right)=$ $\underset{\lim }{\longleftarrow} H^{0}\left(\mathrm{~A}_{\mathrm{dR}} \widehat{\otimes} \mathbb{Q}_{p} / F^{m}\right)=\mathrm{B}_{\mathrm{dR}}^{+} \cdot{ }^{52}$ One has $\mathrm{A}_{\mathrm{dR} n}^{\diamond}=\mathrm{A}_{\mathrm{dR}}^{\diamond} \otimes \mathbb{Z} / p^{n}=\mathrm{A}_{\mathrm{dR}}^{\diamond} \otimes{ }^{L} \mathbb{Z} / p^{n}$.

For any $(V, \bar{V})$, the complex $R \Gamma_{\mathrm{dR}}^{\natural}(V, \bar{V})$ is an $F$-filtered $\mathrm{E}_{\infty}$ filtered $\mathrm{A}_{\mathrm{dR}^{-}}$ algebra, so $\lim _{F} R \Gamma_{\mathrm{dR}}^{\natural}(V, \bar{V})_{n}$ is an $\mathrm{A}_{\mathrm{dR} n}$-algebra, $\lim _{F}\left(R \Gamma_{\mathrm{dR}}^{\natural}(V, \bar{V}) \widehat{\otimes} \mathbb{Q}_{p}\right)$ is a $\mathrm{B}_{\mathrm{dR}}^{+}$-algebra, etc. In 3.5-3.7 below, we will construct the next natural quasi-isomorphisms:

(a) In 3.5, we define an $F$-filtered quasi-isomorphism $\gamma: R \Gamma_{\mathrm{dR}}(V) \otimes_{\bar{K}}$ $\left(\mathrm{A}_{\mathrm{dR}} \widehat{\otimes} \mathbb{Q}_{p}\right) \stackrel{\sim}{\rightarrow} R \Gamma_{\mathrm{dR}}^{\natural}(V, \bar{V}) \widehat{\otimes} \mathbb{Q}_{p}$. The filtration $F$ on $R \Gamma_{\mathrm{dR}}(V)$ is the HodgeDeligne filtration (see [B] 3.4); it is finite and $\operatorname{gr}_{F} R \Gamma_{\mathrm{dR}}(V)$ is a perfect $\bar{K}$ complex. Thus we have

$$
\lim _{F} \gamma: R \Gamma_{\mathrm{dR}}(V) \otimes_{\bar{K}} \mathrm{~B}_{\mathrm{dR}}^{+} \stackrel{\sim}{\rightarrow} \lim _{F}\left(R \Gamma_{\mathrm{dR}}^{\natural}(V, \bar{V}) \widehat{\otimes} \mathbb{Q}_{p}\right)
$$

${ }^{51}$ By Example in 3.6 below, $\mathrm{A}_{\mathrm{dR}}$ is the $J^{[m]}$-topology completion of $\mathrm{A}_{\text {crys }}$.

${ }^{52}$ So $\mathrm{A}_{\mathrm{dR}}^{\diamond} \otimes \mathbb{Q}$ is dense in $\mathrm{B}_{\mathrm{dR}}^{+}$, but not equal to it. 
(b) In 3.6, using the Illusie-Olsson comparison (see 1.9), we construct a natural compatible system of ring homomorphisms $\mathrm{A}_{\operatorname{crys} n} \rightarrow \mathrm{A}_{\mathrm{dR} n}^{\diamond}$ and quasi-isomorphisms $\kappa_{n}: R \Gamma_{\text {crys }}(V, \bar{V})_{(n)} \otimes_{\mathrm{A}_{\text {crys } n}}^{L} \mathrm{~A}_{\mathrm{dR} n}^{\diamond} \stackrel{\sim}{\rightarrow} \lim _{F} R \Gamma_{\mathrm{dR}}^{\natural}(V, \bar{V})_{n}$. The proposition in 2.2 yields $R \Gamma_{\text {crys }}(V, \bar{V}) \otimes_{\mathrm{A}_{\text {crys }}}^{L} \mathrm{~A}_{\mathrm{dR}} \stackrel{\sim}{\rightarrow} \operatorname{holim}_{n} R \Gamma_{\text {crys }}(V$, $\bar{V})_{(n)} \otimes_{\mathrm{A}_{\text {crys } n}}^{L} \mathrm{~A}_{\mathrm{dR} n}^{\diamond}$, so we get

(3.4.3) $\kappa:=\operatorname{holim}_{n} \kappa_{n}: R \Gamma_{\text {crys }}(V, \bar{V}) \otimes_{\mathrm{A}_{\text {crys }}}^{L} \mathrm{~A}_{\mathrm{dR}} \stackrel{\sim}{\rightarrow} \lim _{F}\left(R \Gamma_{\mathrm{dR}}^{\natural}(V, \bar{V}) \widehat{\otimes} \mathbb{Z}_{p}\right)$.

(c) Consider the evident $\mathrm{A}_{\mathrm{dR}} \widehat{\otimes} \mathbb{Z}_{p}$-linear map $R \Gamma_{\mathrm{dR}}^{\natural}(V, \bar{V}) \widehat{\otimes} \mathbb{Z}_{p} \rightarrow$ $R \Gamma_{\mathrm{dR}}^{\natural}(V, \bar{V}) \widehat{\otimes} \mathbb{Q}_{p}$. In 3.7 we prove that the $\mathrm{B}_{\mathrm{dR}}^{+}$-linear extension of its $\lim _{F}$ is a quasi-isomorphism:

$$
\left(\lim _{F}\left(R \Gamma_{\mathrm{dR}}^{\natural}(V, \bar{V}) \widehat{\otimes} \mathbb{Z}_{p}\right)\right) \otimes_{\mathrm{A}_{\mathrm{dR}}^{\diamond}}^{L} \mathrm{~B}_{\mathrm{dR}}^{+} \stackrel{\sim}{\rightarrow} \lim _{F}\left(R \Gamma_{\mathrm{dR}}^{\natural}(V, \bar{V}) \widehat{\otimes} \mathbb{Q}_{p}\right) .
$$

Assuming (a)-(c), let us deduce the theorem. Consider $R \Gamma_{\mathrm{dR}}(V) \otimes_{\bar{K}}$ $\mathrm{B}_{\mathrm{dR}}^{+} \stackrel{\sim}{\rightarrow} R \Gamma_{\text {crys }}(V, \bar{V}) \otimes_{\mathrm{A}_{\text {crys }}}^{L} \mathrm{~B}_{\mathrm{dR}}^{+}$defined as the composition $\left((3.4 .3) \otimes \mathrm{B}_{\mathrm{dR}}^{+}\right)^{-1}$ $(3.4 .4)^{-1}(3.4 .2)$. This is a quasi-isomorphism of presheaves on $\mathcal{V} a r_{\bar{K}}^{\mathrm{ss}}$. Its hsheafification is a quasi-isomorphism of h-sheaves $\mathcal{A}_{\mathrm{dR}} \otimes_{\bar{K}} \mathrm{~B}_{\mathrm{dR}}^{+} \stackrel{\sim}{\rightarrow} \mathcal{A}_{\text {crys }} \otimes_{\mathrm{A}_{\text {crys }}}^{L}$ $\mathrm{B}_{\mathrm{dR}}^{+}$on $\mathcal{V} r_{\bar{K}}$ (we use the notation of [B] 3.4); applying $R \Gamma\left(X_{\mathrm{h}}, \cdot\right)$, we get (3.4.1). The construction is natural, so it commutes with the Galois action. The final property is evident from the constructions of $\rho_{\text {crys }}$ and $\rho_{\mathrm{dR}}$. We are done.

3.5. We consider (a) above. Recall that we have a filtered quasi-isomorphism $R \Gamma_{\mathrm{dR}}^{\natural}(V, \bar{V}) \otimes \mathbb{Q} \stackrel{\sim}{\rightarrow} R \Gamma_{\mathrm{dR}}(V)$, so the evident map $R \Gamma_{\mathrm{dR}}^{\natural}(V, \bar{V}) \rightarrow R \Gamma_{\mathrm{dR}}^{\natural}(V, \bar{V}) \widehat{\otimes}$ $\mathbb{Z}_{p}$ yields the morphism of $F$-filtered $\bar{K}$-algebras $R \Gamma_{\mathrm{dR}}(V) \rightarrow R \Gamma_{\mathrm{dR}}^{\natural}(V, \bar{V}) \widehat{\otimes} \mathbb{Q}_{p}$. Let $\gamma: R \Gamma_{\mathrm{dR}}(V) \otimes_{\bar{K}}\left(\mathrm{~A}_{\mathrm{dR}} \widehat{\otimes} \mathbb{Q}_{p}\right) \rightarrow R \Gamma_{\mathrm{dR}}^{\natural}(V, \bar{V}) \widehat{\otimes} \mathbb{Q}_{p}$ be its $\mathrm{A}_{\mathrm{dR}} \widehat{\otimes}_{\mathbb{Q}_{p}}$-linear extension.

Lemma. $\gamma$ is a filtered quasi-isomorphism.

Proof. By [B] (4.2.1), $\operatorname{gr}_{F}^{m} R \Gamma_{\mathrm{dR}}^{\natural}(V, \bar{V})$ carry a finite filtration $I$. and we have an identification $\operatorname{gr}_{a}^{I} \operatorname{gr}_{F}^{m} R \Gamma_{\mathrm{dR}}^{\natural}(V, \bar{V})=R \Gamma\left(\bar{V}, \Omega_{\langle V, \bar{V}\rangle}^{a}\right)[-a] \otimes_{O_{\bar{K}}}^{L} \operatorname{gr}_{F}^{m-a} \mathrm{~A}_{\mathrm{dR}}$. Here $\Omega_{\langle V, \bar{V}\rangle}^{a}:=\Omega_{(V, \bar{V}) /(\bar{S}, \overline{\mathcal{L}})}^{a}$ are relative differential forms with log singularities. The $I_{a}$ 's are $\operatorname{gr}_{F}{ }_{F} \mathrm{~A}_{\mathrm{dR}}$-submodules of $\operatorname{gr}_{F}^{\circ} R \Gamma_{\mathrm{dR}}^{\natural}(V, \bar{V})$ and the identification is $\mathrm{gr}_{F} \mathrm{~A}_{\mathrm{dR}}$-linear.

Since $R \Gamma\left(\bar{V}, \Omega_{\langle V, \bar{V}\rangle}^{a}\right)$ is a perfect $O_{\bar{K}^{-}}$-complex, applying $\cdot \widehat{\otimes} \mathbb{Q}_{p}$ yields a finite filtration $I$. on $\operatorname{gr}_{F}^{m} R \Gamma_{\mathrm{dR}}^{\natural}(V, \bar{V}) \widehat{\otimes} \mathbb{Q}_{p}$ together with a $\operatorname{gr}_{F} \mathrm{~B}_{\mathrm{dR}}^{+}$-linear identification $\operatorname{gr}_{a}^{I} \operatorname{gr}_{F}^{m} R \Gamma_{\mathrm{dR}}^{\natural}(V, \bar{V}) \widehat{\otimes} \mathbb{Q}_{p}=\underset{m \geq a \geq 0}{\oplus} R \Gamma\left(\bar{V}, \Omega_{\langle V, \bar{V}\rangle}^{a}\right)[-a] \otimes_{O_{\bar{K}}} \operatorname{gr}_{F}^{m-a} \mathrm{~B}_{\mathrm{dR}}^{+}$. 
One also has an evident $\operatorname{gr}_{F}^{\circ} \mathrm{B}_{\mathrm{dR}}^{+}$-linear identification $\operatorname{gr}_{F}^{m}\left(R \Gamma_{\mathrm{dR}}(V) \otimes_{\bar{K}}\right.$

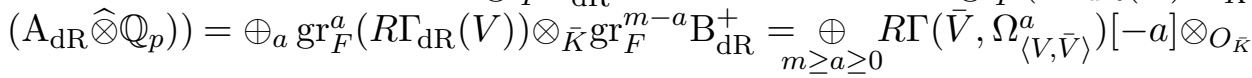
$\operatorname{gr}_{F}^{m-a} \mathrm{~B}_{\mathrm{dR}}^{+}$.

Since $\operatorname{gr}_{F}^{\circ} \gamma$ sends $\operatorname{gr}_{F}^{a} R \Gamma_{\mathrm{dR}}(V)$ to $I_{a} \mathrm{gr}_{F}^{a} R \Gamma_{\mathrm{dR}}^{\natural}(V, \bar{V}) \widehat{\otimes} \mathbb{Q}_{p}=\operatorname{gr}_{F}^{a} R \Gamma_{\mathrm{dR}}^{\natural}(V$, $\bar{V}) \widehat{\otimes} \mathbb{Q}_{p}$, it sends $\operatorname{gr}_{F}^{a} R \Gamma_{\mathrm{dR}}(V) \otimes \operatorname{gr}_{F} \mathrm{~B}_{\mathrm{dR}}^{+}$to $I_{a} \mathrm{gr}_{F}^{+a} R \Gamma_{\mathrm{dR}}^{\natural}(V, \bar{V}) \widehat{\otimes} \mathbb{Q}_{p}$. The lemma follows if we check that $\operatorname{gr}_{F}^{a} R \Gamma_{\mathrm{dR}}(V) \otimes \operatorname{gr}_{F}^{*} \mathrm{~B}_{\mathrm{dR}}^{+} \rightarrow \operatorname{gr}_{a}^{I} \mathrm{gr}_{F}^{++a} R \Gamma_{\mathrm{dR}}^{\natural}(V$, $\bar{V}) \widehat{\otimes} \mathbb{Q}_{p}$ is a quasi-isomorphism. By the above, it suffices to do this for $\cdot=0$. Here it is evident: the projection $R \Gamma_{\mathrm{dR}}^{\natural}(V, \bar{V}) \rightarrow R \Gamma\left(\bar{V}, \Omega_{\langle V, \bar{V}\rangle}\right)$ provides, after applying $\operatorname{gr}_{F}^{a} \cdot \widehat{\otimes} \mathbb{Q}_{p}$, the inverse map.

3.6. Let us consider (b) in 3.4. The $\log$ schemes $(V, \bar{V})_{n} / W_{n}$ satisfy the condition of Remark (i) in 1.9, so we get canonical morphisms

$$
\kappa_{n}: R \Gamma_{\text {crys }}(V, \bar{V})_{(n)} \rightarrow \lim _{F} R \Gamma_{\mathrm{dR}}^{\natural}(V, \bar{V})_{n} .
$$

Here $\kappa_{n}$ is projective limit of $\kappa_{m, n}: R \Gamma_{\text {crys }}(V, \bar{V})_{(n)} \rightarrow R \Gamma_{\mathrm{dR}}^{\natural}(V, \bar{V})_{n} / F^{m}$, where $\kappa_{m, n}$ is the composition $R \Gamma_{\text {crys }}(V, \bar{V})_{(n)} \stackrel{\sim}{\rightarrow} R \Gamma\left(\left((V, \bar{V})_{n} / W_{n}\right)_{\text {crys }}, \mathcal{O}\right) \rightarrow$ $R \Gamma\left(\left((V, \bar{V})_{n} / W_{n}\right), \mathcal{O} / \mathcal{J}^{[m]}\right) \stackrel{\sim}{\rightarrow} R \Gamma_{\mathrm{dR}}^{\natural}(V, \bar{V})_{n} / F^{m}$, the first $\stackrel{\sim}{\rightarrow}$ comes from Remark (i) in 1.12, the second $\stackrel{\sim}{\rightarrow}$ is the inverse to the Illusie-Olsson isomorphism (1.9.2).

Example. For $(V, \bar{V})=\operatorname{Spec}\left(\bar{K}, O_{\bar{K}}\right)$, our $\kappa_{n}$ is a canonical map $\mathrm{A}_{\text {crys } n} \rightarrow$ $\mathrm{A}_{\mathrm{dR} n}$. By Illusie-Olsson (see 1.9), it identifies $\mathrm{A}_{\operatorname{crys} n} / J_{n}^{[m]}$ with $\mathrm{A}_{\mathrm{dR} n} / F^{m}$, where $J_{n}$ is the kernel of the projection $\mathrm{A}_{\text {crys } n} \rightarrow \hat{O}_{\bar{K}} / p^{n}$. Thus $\mathrm{A}_{\mathrm{dR}}^{\diamond}$ is the completion of $\mathrm{A}_{\text {crys }}$ with respect to the $J^{[m]}$-topology.

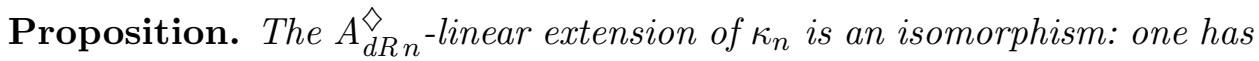

$$
R \Gamma_{\text {crys }}(V, \bar{V})_{(n)} \otimes_{A_{\text {crys } n}}^{L} A_{d R n}^{\diamond} \stackrel{\sim}{\rightarrow} \lim _{F} R \Gamma_{d R}(V, \bar{V})_{n}
$$

Proof. By Proposition in 2.2, the 1.h.s. in (3.6.2) equals holim ${ }_{m} R \Gamma_{\text {crys }}(V$, $\bar{V})_{(n)} \otimes_{\mathrm{A}_{\text {crys } n}}^{L}\left(\mathrm{~A}_{\text {crys } n} / J_{n}^{[m]}\right) \stackrel{\sim}{\rightarrow} \operatorname{holim}_{m} R \Gamma\left(\left((V, \bar{V})_{n} /\left(\bar{S}_{n}, \mathrm{~A}_{\text {crys } n} / J_{n}^{[m]}\right)\right)_{\text {crys }}\right.$, $\left.\mathcal{O}_{\bar{V}_{n} /\left(\mathrm{A}_{\text {crys } n} / J_{n}^{[m]}\right)}\right)$ by base change and (1.18.1). Since $(V, \bar{V})_{n}$ is $\log$ smooth over $(\bar{S}, \overline{\mathcal{L}})_{n}$, the Hodge-pd filtration on $\Omega_{\bar{V}_{n} /\left(\mathrm{A}_{\text {crys } n} / J_{n}^{[m]}\right)}$ is finite (in fact, $F^{N} \Omega_{\bar{V}_{n} /\left(\mathrm{A}_{\text {crys } n} / J_{n}^{[m]}\right)}$ vanishes for $\left.N \geq \operatorname{dim} V+m\right)$, so the above completion equals the completion for the Hodge-pd filtration, which is the r.h.s. of (3.6.2) by the Illusie-Olsson theorem in 1.9. 
3.7. Consider finally (c) in 3.4. Using (3.4.2) and (3.4.3), the map (3.4.4) can be rewritten as $\phi: R \Gamma_{\text {crys }}(V, \bar{V}) \otimes_{\mathrm{A}_{\text {crys }}}^{L} \mathrm{~B}_{\mathrm{dR}}^{+} \rightarrow R \Gamma_{\mathrm{dR}}(V) \otimes_{\bar{K}} \mathrm{~B}_{\mathrm{dR}}^{+}$. Both terms are perfect $\mathrm{B}_{\mathrm{dR}}^{+}$-complexes (see Proposition in 2.2) and $\mathrm{B}_{\mathrm{dR}}^{+}$is a dvr, so to prove that $\phi$ is a quasi-isomorphism it suffices to check that its pullback $\phi_{\mathbb{C}_{p}}: R \Gamma_{\text {crys }}(V, \bar{V}) \otimes_{\mathrm{A}_{\text {crys }}}^{L} \mathbb{C}_{p} \rightarrow R \Gamma_{\mathrm{dR}}(V) \otimes_{\bar{K}} \mathbb{C}_{p}$ to $\mathrm{B}_{\mathrm{dR}}^{+} / \mathfrak{m}_{\mathrm{dR}}=\mathbb{C}_{p}$ is a quasiisomorphism. We use 2.2 and the notation in loc. cit. By (1.18.1) and base change, one has $R \Gamma_{\text {crys }}(V, \bar{V}) \otimes_{\mathrm{A}_{\text {crys }}}^{L} \hat{O}_{\bar{K}} \stackrel{\sim}{\rightarrow} R \Gamma\left(Z, \Omega_{(Z, \mathcal{M}) /(S, \mathcal{L})}\right) \otimes_{O_{K}}^{L} \hat{O}_{\bar{K}}$, hence $R \Gamma_{\text {crys }}(V, \bar{V}) \otimes_{\mathrm{A}_{\text {crys }}}^{L} \mathbb{C}_{p} \stackrel{\sim}{\rightarrow} R \Gamma_{\mathrm{dR}}(V) \otimes_{\bar{K}} \mathbb{C}_{p}$. Comparing it with the definition of $\kappa$ in (3.4.2), we see that this identification equals $\phi_{\mathbb{C}_{p}}$, and we are done.

\section{Index of notation}

$(Z, \mathcal{M}),(Z, \mathcal{M})^{\mathrm{int}}, \mathcal{M}^{\mathrm{gr}}, \mathbb{A}_{(S, \mathcal{L})}^{(1)} 1.1 ; S^{\sharp}, G^{\sharp} 1.2 ; \mathcal{T}_{S^{\sharp}}, \mathcal{C}_{S^{\sharp}} 1.3 ; \mathcal{O}_{Z / S}, \mathcal{J}_{Z / S}$, $u_{Z / S}^{\log }, f_{\text {crys }},\left((Z, \mathcal{M}) / S^{\sharp}\right)_{\text {crys }},(Z / S)_{\text {crys }}^{\log 1.5 ;}\left(U, T_{*}\right), \mathcal{C} \cdot \mathcal{F} 1.6 ; \Omega_{(U, T) / S}, \Omega_{Z / S}$, $F^{m}, \nabla_{(Z, P)} 1.7 ; L \Omega_{(Z, \mathcal{M}) /(S, \mathcal{L})} 1.9 ; F r_{T}, F r_{T}^{\prime}, C 1.10 ; D^{\mathrm{pcr}} 1.11 ; i_{n}, \mathcal{F}_{(n)}$, $(Z, \mathcal{M})_{\text {crys }}, \quad(Z, \mathcal{M})_{\text {crys }(n)}, \quad R \Gamma_{\text {crys }}(Z, \mathcal{M})_{(n)}, \quad R \Gamma_{\text {crys }}(Z, \mathcal{M}), \quad H_{\text {crys }}^{i}(Z, \mathcal{M})$, $(Z, \mathcal{M})_{n} 1.12 ; R_{\varphi}$-mod, $\operatorname{Hom}_{R_{\varphi}}^{\natural}, D_{\varphi}(R), R_{\varphi}^{\mathrm{prf}}-\bmod , D_{\varphi}^{\mathrm{prf}}(R)^{\mathrm{nd}} 1.13 ; D_{\varphi}^{\mathrm{pcr}}(Z / S)$, $D_{\varphi}^{\mathrm{pcr}}(Z / S)^{\mathrm{nd}}, D_{\varphi}\left((Z / S)_{\text {crys }}^{\log }, \mathcal{O}_{Z / S}\right) 1.14 ; K_{0}, \epsilon_{Y}, D_{\varphi}^{\mathrm{pcr}}(Y)^{\mathrm{nd}}, \operatorname{Hom}_{\varphi, N}^{\natural},(\varphi, N)-$ $\bmod ,(\varphi, N)^{\mathrm{eff}}{ }_{-} \bmod , D_{\varphi, N}\left(K_{0}\right), D_{\varphi, N}\left(K_{0}\right)^{\mathrm{eff}} 1.15 ; R \Gamma_{\mathrm{HK}}\left(Z_{1}^{0}, \mathcal{M}_{1}^{0}\right), A_{\mathbb{Q}}^{\tau}, \iota_{\mathrm{dR}}$, $R \Gamma_{\mathrm{HK}}\left(Z_{1}^{0}, \mathcal{M}_{1}^{0}\right)^{\tau}{ }_{A_{\mathbb{Q}}} 1.16 ; \mathrm{A}_{\text {crys }}, J_{\text {crys }}, E_{\text {crys }}, \mathcal{L}_{\text {crys }}, \mathcal{L}_{\varphi} 1.17 ; R \Gamma_{\mathrm{HK}}\left(Z_{1}^{-}, \mathcal{M}_{1}^{-}\right)$, $\mathrm{B}_{\text {crys }}^{+}, \iota_{\text {crys }}, \mathrm{B}_{\text {st }}^{+}, K_{0}^{\mathrm{nr}} 1.18 ; \cdot \widehat{\otimes} \mathbb{Z}_{p}, \cdot \widehat{\otimes} \mathbb{Q}_{p} 2.1 ; \mathcal{V} a r_{\bar{K}}^{\mathrm{ss}}, R \Gamma_{\text {crys }}(V, \bar{V}), R \Gamma_{\text {crys }}(V, \bar{V})_{(n)}$ $2.2 ; \mathcal{A}_{\text {crys }} 2.3 ; R \Gamma_{\text {crys }}(X), H_{\text {crys }}^{n}(X) 2.4 ; \mathcal{A}_{\mathrm{HK}}, R \Gamma_{\mathrm{HK}}(X), H_{\mathrm{HK}}^{n}(X), R \Gamma_{\mathrm{dR}}(X)$, $R \Gamma_{\mathrm{HK}}(X)_{\mathrm{B}_{\text {crys }}^{+}}^{\tau}, R \Gamma_{\mathrm{HK}}(X)_{\bar{K}}^{\tau} 2.5 ; \rho_{\text {crys }}, \rho_{\mathrm{HK}} 3.1 ; R \Gamma_{\mathrm{dR}}^{\natural}(V, \bar{V}), \lim _{F}, \mathrm{~A}_{\mathrm{dR}}, \mathrm{A}_{\mathrm{dR}}^{\diamond}$ 3.4 .

\section{References}

[B] A. Beilinson, p-adic periods and derived de Rham cohomology, J. Amer. Math. Soc. 25 (2012), no. 3, 715-738.

[Ber] P. Berthelot, Cohomologie cristalline des schémas de characteristique $p>0$, Lect. Notes in Math. 407, Springer-Verlag, 1974.

[BO1] P. Berthelot, A. Ogus, Notes on crystalline cohomology, Math. Notes 21, Princeton University Press, 1978.

[BO2] P. Berthelot, A. Ogus, F-isocrystals and de Rham cohomology I, Invent. Math. 72 (1983), 159-199.

[Bh1] B. Bhatt, p-divisibility for coherent cohomology, math. AG 1204.5831 (2012). 
[Bh2] B. Bhatt, p-adic derived de Rham cohomology, math. AG 1204.6560 (2012).

[BhdJ] B. Bhatt, A. J. de Jong, Crystalline cohomology and de Rham cohomology, math. AG 1110.5001 (2011).

[D] P. Deligne, Théorèmes de finitude en cohomologie $\ell$-adique, Cohomologie étale (SGA $4 \frac{1}{2}$ ), Lect. Notes in Math. 569, Springer-Verlag, 1977, pp. 233-251.

[Fa] G. Faltings, Integral crystalline cohomology over very ramified valuation rings, J. Amer. Math. Soc. 12 (1999), no. 1, 117-144.

[F1] J.-M. Fontaine, Le corps de périodes p-adiques, Périodes p-adiques, Astérisque 223, SMF, 1994, pp. 59-101.

[F2] J.-M. Fontaine, Représentations p-adiques semi-stables, Périodes padiques, Astérisque 223, SMF, 1994, pp. 113-184.

[GR] O. Gabber, L. Ramero, Foundations for almost ring theory, math. AG 0201175 (2002).

[G] A. Grothendieck, Site et topos étales d'un schéma, Théorie des topos et cohomologie étale de schémas (SGA 4), Tome II, Lect. Notes in Math. 270, Springer-Verlag, 1972, pp. 341-365.

[HS] V. Hinich, V. Schechtman, On homotopy limit of homotopy algebras, K-theory, Arithmetic and Geometry, Yu. I. Manin (Ed.), Lect. Notes in Math. 1289, Springer-Verlag, 1987, pp. 240-264.

[HK] O. Hyodo, K. Kato, Semi-stable reduction and crystalline cohomology with logarithmic poles, Périodes p-adiques, Astérisque 223, SMF, 1994, pp. 221-268.

[Ill1] L. Illusie, Complexe cotangent et déformations I, Lect. Notes in Math. 239, Springer-Verlag, 1971.

[Ill2] L. Illusie, Complexe cotangent et déformations II, Lect. Notes in Math. 283, Springer-Verlag, 1972.

[Ill3] L. Illusie, Pre-notes for Sapporo seminar (March 2011).

[K1] K. Kato, Logarithmic structures of Fontaine-Illusie, Algebraic Analysis, Geometry, and Number Theory, The Johns Hopkins University Press, 1988, pp. 191-224.

[K2] K. Kato, Semi-stable reduction and p-adic étale cohomology, Périodes p-adiques, Astérisque 223, SMF, 1994, pp. 269-293. 
[KS] K. Kato, T. Saito, On the conductor formula of Bloch, Publ. Math. IHES 100 (2004), 5-151.

[Ka] N. Katz, Travaux de Dwork, Séminaire Bourbaki 24ème année (1971/1972), Exp. No. 409, Lect. Notes in Math. 317, Springer, 1973, pp. $167-200$.

[Ol1] M. Olsson, The logarithmic cotangent complex, Math. Ann. 333 (2005), 859-931.

[O12] M. Olsson, Crystalline cohomology of algebraic stacks and HyodoKato cohomology, Astérisque, vol. 316, SMF, 2007.

[Og1] A. Ogus, Lectures on logarithmic algebraic geometry, http://math. berkeley.edu/ ${ }^{\sim}$ ogus/.

[Og2] A. Ogus, Logarithmic de Rham cohomology, preprint (1998).

[S] M. Saito, Induced $\mathcal{D}$-modules and differential complexes, Bull. SMF 117 (1989), no. 3, 361-387.

[SV] A. Suslin, V. Voevodsky, Singular homology of abstract algebraic varieties, Inv. Math. 123 (1996), 61-94.

\author{
A. Beilinson \\ Department of Mathematics \\ UNIVERSITY OF CHICAGO \\ ChicAGo, IL 60637 \\ USA \\ E-mail address: sasha@math.uchicago.edu
}

Received November 14, 2011 